\title{
A Rapid Synthesis of the Biotin Core Through a Tandem Michael Reaction
}

\author{
Kyungsoo $O h^{*}$ \\ Department of Chemistry and Chemical Biology, Indiana University Purdue University \\ Indianapolis (IUPUI), Indianapolis, Indiana 46202
}

oh@chem.iupui.edu

Table of Contents:

Experimental Section

S3-S22

${ }^{1} \mathrm{H}$ NMR Spectrum of $(\boldsymbol{Z})-\mathbf{8}$

$\mathrm{S} 23$

${ }^{13} \mathrm{C}$ NMR Spectrum of $(\boldsymbol{Z})-\mathbf{8}$

S24

${ }^{1} \mathrm{H}$ NMR Spectrum of $(\boldsymbol{E})-\mathbf{8}$

S25

${ }^{13} \mathrm{C}$ NMR Spectrum of $(\boldsymbol{E})-8$

S26

${ }^{1} \mathrm{H}$ NMR Spectrum of 9

S27

${ }^{13} \mathrm{C}$ NMR Spectrum of 9

S28

${ }^{1} \mathrm{H}$ NMR Spectrum of $\mathbf{1 0}$

S29

${ }^{13} \mathrm{C}$ NMR Spectrum of $\mathbf{1 0}$

S30

${ }^{1} \mathrm{H}$ NMR Spectrum of $\mathbf{1 1}$

S31

${ }^{13} \mathrm{C}$ NMR Spectrum of $\mathbf{1 1}$

S32

${ }^{1}$ H NMR Spectrum of trans-Sulfoxide 12

S33

${ }^{13} \mathrm{C}$ NMR Spectrum of trans-Sulfoxide 12

S34

${ }^{1} \mathrm{H}$ NMR Spectrum of cis-Sulfoxide 12

S35

${ }^{13} \mathrm{C}$ NMR Spectrum of cis-Sulfoxide 12

S36

${ }^{1} \mathrm{H}$ NMR Spectrum of $\mathbf{1 3}$

S37

${ }^{13} \mathrm{C}$ NMR Spectrum of $\mathbf{1 3}$

S38

${ }^{1} \mathrm{H}$ NMR Spectrum of $\mathbf{1 4}$

S39

${ }^{13} \mathrm{C}$ NMR Spectrum of 14

S40

${ }^{1} \mathrm{H}$ NMR Spectrum of cis-Sulfoxide 14

S41

${ }^{13} \mathrm{C}$ NMR Spectrum of cis-Sulfoxide 14

S42

$\begin{array}{ll}{ }^{1} \mathrm{H} \text { NMR Spectrum of 2,3-cis-Sulfone } 16 & \text { S43 }\end{array}$

$\begin{array}{ll}{ }^{13} \mathrm{C} \text { NMR Spectrum of 2,3-cis-Sulfone } 16 & \text { S44 }\end{array}$

$\begin{array}{ll}{ }^{1} \mathrm{H} \text { NMR Spectrum of 2,3-trans-Sulfone 16 } & \text { S45 }\end{array}$

${ }^{13} \mathrm{C}$ NMR Spectrum of 2,3-trans-Sulfone $16 \quad$ S46

$\begin{array}{ll}{ }^{1} \mathrm{H} \text { NMR Spectrum of 2,3-cis-Sulfoxide 16} & \text { S47 }\end{array}$

$\begin{array}{ll}{ }^{13} \mathrm{C} \text { NMR Spectrum of 2,3-cis-Sulfoxide } 16 & \text { S48 }\end{array}$

$\begin{array}{ll}{ }^{1} \text { H NMR Spectrum of 2,3-trans-Sulfoxide 16 } & \text { S49 }\end{array}$

${ }^{13} \mathrm{C}$ NMR Spectrum of 2,3-trans-Sulfoxide $16 \quad$ S50

${ }^{1} \mathrm{H}$ NMR Spectrum of 2,3-trans-Sulfoxide 16a $\quad$ S51

${ }^{13} \mathrm{C}$ NMR Spectrum of 2,3-trans-Sulfoxide 16a $\quad$ S52

${ }^{1} \mathrm{H}$ NMR Spectrum of 2,3-cis-Sulfoxide 16a $\quad$ S53

${ }^{13} \mathrm{C}$ NMR Spectrum of 2,3-cis-Sulfoxide 16a $\quad$ S54 
${ }^{1} \mathrm{H}$ NMR Spectrum of 2,3-cis-Sulfone 17

${ }^{13} \mathrm{C}$ NMR Spectrum of 2,3-cis-Sulfone 17

${ }^{1} \mathrm{H}$ NMR Spectrum of 2,3-trans-Sulfone 17

${ }^{13} \mathrm{C}$ NMR Spectrum of 2,3-trans-Sulfone 17

${ }^{1} \mathrm{H}$ NMR Spectrum of 2,3-trans-Sulfone 4

${ }^{13} \mathrm{C}$ NMR Spectrum of 2,3-trans-Sulfone 4

${ }^{1} \mathrm{H}$ NMR Spectrum of 2,3-cis-Sulfoxide $\mathbf{6 a}$

${ }^{13} \mathrm{C}$ NMR Spectrum of 2,3-cis-Sulfoxide 6a

S62

${ }^{1} \mathrm{H}$ NMR Spectrum of 2,3-trans-Sulfoxide 6

S63

${ }^{13} \mathrm{C}$ NMR Spectrum of 2,3-trans-Sulfoxide 6

S64

${ }^{1} \mathrm{H}$ NMR Spectrum of 2,3-cis-Sulfoxide 5a

S65

${ }^{13} \mathrm{C}$ NMR Spectrum of 2,3-cis-Sulfoxide 5a

S66

${ }^{1} \mathrm{H}$ NMR Spectrum of 2,3-trans-Sulfoxide 5

S67

${ }^{13} \mathrm{C}$ NMR Spectrum of 2,3-trans-Sulfoxide 5

S68

${ }^{1} \mathrm{H}$ NMR Spectrum of 2,3-cis-Sulfide 18

S69

${ }^{13} \mathrm{C}$ NMR Spectrum of 2,3-cis-Sulfide 18

S70

${ }^{1} \mathrm{H}$ NMR Spectrum of 2,3-trans-Sulfide 2

${ }^{13} \mathrm{C}$ NMR Spectrum of 2,3-trans-Sulfide 2

S71

S72 


\section{Data of products}

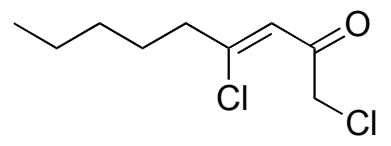

(Z)-(8)

To a stirred suspension of aluminium chloride $(9.50 \mathrm{~g}, 71 \mathrm{mmol})$ in dry dichloromethane $(150 \mathrm{ml})$ at $0{ }^{\circ} \mathrm{C}$, was added a mixture of 1-heptyne $(8.5 \mathrm{ml}, 65 \mathrm{mmol})$ and chloro actetyl chloride $(5.2 \mathrm{ml}, 65 \mathrm{mmol})$ dropwise over 10 minutes. The cooling bath was removed and the reaction mixture was warmed up to ambient temperature over one hour. The mixture was poured into a mixture of brine and ice. The mixture was stirred for two hours at ambient temperature and extracted using dichloromethane $(100 \mathrm{ml} \times 3)$. The combined organic layers were washed with brine and dried over magnesium sulfate and concentrated in vacuo. The residue was purified by flash chromatography on silica gel (eluent $95 / 5$ hexanes/diethyl ether) to give $(Z)$-alkene $(3.37 \mathrm{~g}, 25 \%)$ and $(E)$-alkene $(7.43 \mathrm{~g}, 55 \%)$ as yellowish liquids. The assignment of stereochemistry of the product was based on the work of Martens.

$\mathrm{R}_{\mathrm{f}}=0.41\left(3: 97 \mathrm{Et}_{2} \mathrm{O} / \mathrm{Hex}\right) ;$

IR (neat) 2957, 2931, 2862, 1692, 1599, 1461;

${ }^{1} \mathrm{H}$ NMR $\left(500 \mathrm{MHz}, \mathrm{CDCl}_{3}\right): 6.61(1 \mathrm{H}, \mathrm{s}), 4.08(2 \mathrm{H}, \mathrm{s}), 2.96(2 \mathrm{H}, \mathrm{t}, 7.6 \mathrm{~Hz}), 1.60(2 \mathrm{H}, \mathrm{m})$, $1.32(4 \mathrm{H}, \mathrm{m}), 0.89(3 \mathrm{H}, \mathrm{t}, 7.1 \mathrm{~Hz})$;

${ }^{13} \mathrm{C}$ NMR $\left(125 \mathrm{MHz}, \mathrm{CDCl}_{3}\right): 189.0,161.1,121.6,49.0,36.5,30.9,27.3,22.3,13.8$;

HRMS calcd for $\mathrm{C}_{9} \mathrm{H}_{15} \mathrm{O}_{1} \mathrm{Cl}_{2} 209.0500$, found $209.0509\left(\mathrm{MH}^{+}\right)$

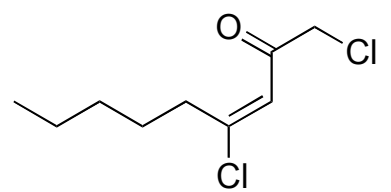

$(E)-(8)$

$\mathrm{R}_{\mathrm{f}}=0.17\left(3: 97 \mathrm{Et}_{2} \mathrm{O} / \mathrm{Hex}\right)$;

IR (neat) 2957, 2931, 2862, 1715, 1697, 1610, 1463;

${ }^{1} \mathrm{H}$ NMR (500MHz, $\left.\mathrm{CDCl}_{3}\right): 6.48(1 \mathrm{H}, \mathrm{s}), 4.25(2 \mathrm{H}, \mathrm{s}), 2.47(2 \mathrm{H}, \mathrm{t}, 7.4 \mathrm{~Hz}), 1.63(2 \mathrm{H}, \mathrm{m})$, $1.30(4 \mathrm{H}, \mathrm{m}), 0.89(3 \mathrm{H}, \mathrm{t}, 7.1 \mathrm{~Hz})$;

${ }^{13} \mathrm{C}$ NMR (125MHz, $\left.\mathrm{CDCl}_{3}\right):$ 189.2, 151.5, 120.6, 49.0, 41.6, 30.6, 26.9, 22.2, 13.8;

${ }^{1}$ Martens, H.; Hoornaert, G.; Toppet, S. Tetrahedron 1973, 29, 4249. 
HRMS calcd for $\mathrm{C}_{9} \mathrm{H}_{15} \mathrm{O}_{1} \mathrm{Cl}_{2} 209.0500$, found $209.0503\left(\mathrm{MH}^{+}\right)$

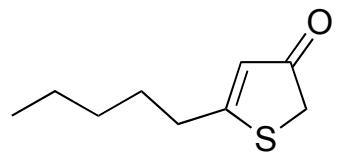

(9)

To a stirred sodium hydrosulfide hydrate $(6.8 \mathrm{~g}, 121 \mathrm{mmol})$ at ambient temperature, was added the alkenes $8(10.14 \mathrm{~g}, 48 \mathrm{mmol})$ dropwise to maintain self-refluxing over 2 minutes. The reaction was normally over in 5 minutes as evidenced by thin layer chromatography. The reaction was diluted with diethyl ether $(200 \mathrm{ml})$ and filtered through Celite. After solvent removal under reduced pressure, the residue was purified by flash chromatography on silica gel (eluent 90/10 hexanes/diethyl ether) to give thiophen-3-one 9 as a reddish oil $(5.47 \mathrm{~g}, 67 \%)$.

$\mathrm{R}_{\mathrm{f}}=0.20\left(20: 80 \mathrm{Et}_{2} \mathrm{O} / \mathrm{Hex}\right) ;$

IR (neat) 2957, 2928, 2857, 1656, 1564, 1460;

${ }^{1} \mathrm{H}$ NMR (500MHz, $\left.\mathrm{CDCl}_{3}\right): 6.00(1 \mathrm{H}, \mathrm{s}), 3.62(2 \mathrm{H}, \mathrm{s}), 2.61(2 \mathrm{H}, \mathrm{t}, 7.6 \mathrm{~Hz}), 1.65(2 \mathrm{H}, \mathrm{m})$, $1.34(4 \mathrm{H}, \mathrm{m}), 0.89(3 \mathrm{H}, \mathrm{t}, 6.6 \mathrm{~Hz})$;

${ }^{13} \mathrm{C}$ NMR (125MHz, $\mathrm{CDCl}_{3}$ ): 202.8, 185.7, 120.9, 40.8, 33.9, 31.2, 28.3, 22.4, 13.9;

HRMS calcd for $\mathrm{C}_{9} \mathrm{H}_{14} \mathrm{O}_{1} \mathrm{~S}_{1} 170.0765$, found $170.0759\left(\mathrm{M}^{+}\right)$

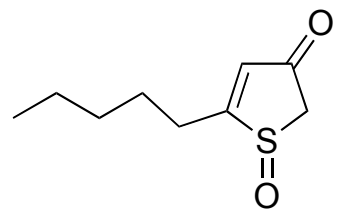

(10)

To a stirred solution of thiophen-3-one $9(1.00 \mathrm{~g}, 5.87 \mathrm{mmol})$ in dry dichloromethane $(100 \mathrm{ml})$ at $-20{ }^{\circ} \mathrm{C}$, was added $m$-chloroperbenzoic acid $(1.45 \mathrm{~g}, 5.87 \mathrm{mmol})$ portionwise. The resulting solution was stirred for 5 minutes at $0{ }^{\circ} \mathrm{C}$ and quenched with saturated sodium bicarbonate solution and extracted with ethyl acetate $(50 \mathrm{ml} \times 3)$. The combined organic layers were washed with brine and dried over magnesium sulfate and concentrated in vacuo. The residue was purified by flash chromatography on silica gel (eluent 40/60 ethyl acetate/hexanes) to give sulfoxide $\mathbf{1 0}$ as a white solid (0.85g, 78\%).

Mp: $59-61{ }^{\circ} \mathrm{C}$

$\mathrm{R}_{\mathrm{f}}=0.17\left(50: 50 \mathrm{Et}_{2} \mathrm{O} / \mathrm{Hex}\right) ;$ 
IR (neat); 2957, 2930, 2860, 1713, 1612, 1465, 1375, 1293, 1254, 1200, 1088, 1044, 891, 845 ;

${ }^{1} \mathrm{H}$ NMR $\left(500 \mathrm{MHz}, \mathrm{CDCl}_{3}\right): 6.39(1 \mathrm{H}, \mathrm{t}, 1.4 \mathrm{~Hz}), 4.05(1 \mathrm{H}, \mathrm{d}, 17.0 \mathrm{~Hz}), 3.40(1 \mathrm{H}, \mathrm{d}$, $17.0 \mathrm{~Hz}), 2.74(2 \mathrm{H}, \mathrm{m}), 1.70(2 \mathrm{H}, \mathrm{m}), 1.34(4 \mathrm{H}, \mathrm{m}), 0.86(3 \mathrm{H}, \mathrm{t}, 7.1 \mathrm{~Hz})$;

${ }^{13} \mathrm{C}$ NMR (125MHz, $\mathrm{CDCl}_{3}$ ): 193.8, 181.6, 130.3, 59.9, 31.2, 29.0, 27.0, 22.2, 13.8;

HRMS calcd for $\mathrm{C}_{9} \mathrm{H}_{13} \mathrm{O}_{1} \mathrm{~S}_{1}$ 169.0687, found $169.0687\left([\mathrm{M}-\mathrm{OH}]^{+}\right)$

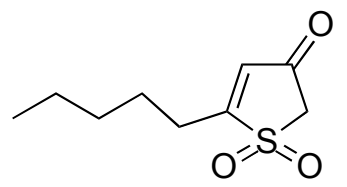

(11)

\section{Oxidation using ${ }^{m} C P B A$}

To a stirred solution of thiophen-3-one $9(0.556 \mathrm{~g}, 3.26 \mathrm{mmol})$ in dry dichloromethane $(50 \mathrm{ml})$ at $0{ }^{\circ} \mathrm{C}$, was added $m$-chloroperbenzoic acid $(2.42 \mathrm{~g}, 9.79 \mathrm{mmol})$ portionwise. The resulting solution was warmed up to ambient temperature over one hour and stirred for another two hours. The reaction was quenched with saturated potassium carbonate solution and extracted with dichloromethane $(50 \mathrm{ml} \times 3)$. The combined organic layers were washed with brine and dried over magnesium sulfate and concentrated in vacuo. The residue was purified by flash chromatography on silica gel (eluent 90/10 hexanes/diethyl ether) to give sulfone 11 as a white solid $(0.535 \mathrm{~g}, 81 \%)$.

\section{Oxidation using Oxone $e^{\circledast}$}

To a stirred solution of thiophen-3-one $9(0.500 \mathrm{~g}, 2.93 \mathrm{mmol})$ in methanol and water $(24 \mathrm{ml}(1: 1))$ at ambient temperature, was added Oxone ${ }^{\circledR}(5.40 \mathrm{~g}, 7.17 \mathrm{mmol})$ portionwise. The resulting mixture was stirred for five hours in which tlc monitoring indicated the consumption of all starting material. The methanol was removed under reduced pressure, and the mixture was partitioned between diethyl ether and water. Extraction using diethyl ether $(50 \mathrm{ml} \times 3)$ followed by washing with brine and drying over magnesium sulfate gave a crude product after removing the solvent in vacuo. The residue was purified by flash chromatography on silica gel (eluent 90/10 hexanes/diethyl ether) to give sulfone 11 as a white solid $(0.47 \mathrm{~g}, 80 \%)$.

Mp: $91-92^{\circ} \mathrm{C}$

$\mathrm{R}_{\mathrm{f}}=0.25\left(20: 80 \mathrm{Et}_{2} \mathrm{O} / \mathrm{Hex}\right) ;$

IR (neat) 3087, 3061, 2999, 2953, 2869, 1717, 1617, 1466, 1422, 1371, 1319, 1302, $1266,1202,1137,906,864,737,704$; 
${ }^{1} \mathrm{H}$ NMR (500MHz, $\left.\mathrm{CDCl}_{3}\right): 6.43(1 \mathrm{H}, \mathrm{s}), 3.79(2 \mathrm{H}, \mathrm{s}), 2.70(2 \mathrm{H}, \mathrm{t}, 7.6 \mathrm{~Hz}), 1.74(2 \mathrm{H}, \mathrm{m})$, $1.39(4 \mathrm{H}, \mathrm{m}), 0.92(3 \mathrm{H}, 7.1 \mathrm{~Hz})$;

${ }^{13} \mathrm{C} \mathrm{NMR}\left(125 \mathrm{MHz}, \mathrm{CDCl}_{3}\right): 187.5,170.4,129.9,54.9,31.1,26.5,24.3,22.1,13.7$;

HRMS calcd for $\mathrm{C}_{9} \mathrm{H}_{14} \mathrm{O}_{3} \mathrm{~S}_{1} 203.0742$, found $203.0745\left(\mathrm{MH}^{+}\right)$

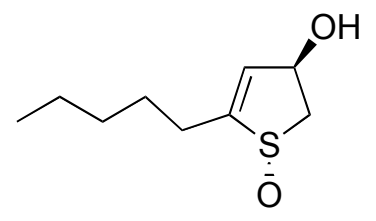

trans-Sulfoxide (12)

To a stirred solution of the sulfoxide $10(3.40 \mathrm{~g}, 18.2 \mathrm{mmol})$ in dry methanol $(30 \mathrm{ml})$ at -10 ${ }^{\circ} \mathrm{C}$, was added sodium borohydride $(0.90 \mathrm{~g}, 23.7 \mathrm{mmol})$ in portionwise. The resulting solution was stirred for 90 minutes at $0 \sim 5{ }^{\circ} \mathrm{C}$ then solvent was removed under reduced pressure. The resulting mixture was partitioned between water and ethyl acetate and extracted with ethyl acetate $(30 \mathrm{ml} \times 3)$. The combined organic layers were washed with brine and dried over magnesium sulfate and concentrated in vacuo. The residue was purified by flash chromatography on silica gel (eluent $2 / 98$ methanol/dichloromethane) to give trans-sulfoxide $12(3.00 \mathrm{~g}, 88 \%)$ and cis-sulfoxide $12(0.21 \mathrm{~g}, 6 \%)$ as yellowish oil. The stereochemistry of the product was retrospectively assigned after converting $\mathbf{1 2}$ to urea 5 and $\mathbf{6}$.

$\mathrm{R}_{\mathrm{f}}=0.25\left(5: 95 \mathrm{MeOH} / \mathrm{CH}_{2} \mathrm{Cl}_{2}\right)$;

IR (neat); 3300, 2956, 2931, 2858, 1458, 1430, 1378, 1322, 1258, 1006;

${ }^{1} \mathrm{H}$ NMR (500MHz, $\left.\mathrm{CDCl}_{3}\right): 6.17(1 \mathrm{H}, \mathrm{s}), 4.80(1 \mathrm{H}, \mathrm{s}), 4.75(1 \mathrm{H}, \mathrm{s}), 3.98(1 \mathrm{H}, \mathrm{dd}$, $13.7 \mathrm{~Hz}, 6.8 \mathrm{~Hz}), 2.81(1 \mathrm{H}, \mathrm{dd}, 13.7 \mathrm{~Hz}, 4.5 \mathrm{~Hz}), 2.47(2 \mathrm{H}, \mathrm{t}, 7.5 \mathrm{~Hz}), 1.64-1.54(2 \mathrm{H}, \mathrm{m})$, $1.31(4 \mathrm{H}, \mathrm{m}), 0.87(3 \mathrm{H}, \mathrm{t}, 6.9 \mathrm{~Hz})$;

${ }^{13} \mathrm{C}$ NMR (125MHz, $\left.\mathrm{CDCl}_{3}\right): 150.8,134.3,72.5,59.8,31.1,27.5,26.8,22.2,13.8$;

HRMS calcd for $\mathrm{C}_{9} \mathrm{H}_{17} \mathrm{O}_{2} \mathrm{~S}_{1} 189.0949$, found $189.0944\left(\mathrm{MH}^{+}\right)$<smiles>CCCCCC1=C[C@H](O)CS1=O</smiles>

cis-Sulfoxide (12)

$\mathrm{R}_{\mathrm{f}}=0.20\left(5: 95 \mathrm{MeOH} / \mathrm{CH}_{2} \mathrm{Cl}_{2}\right)$; 
IR (neat); 3321, 2956, 2930, 2859, 1465, 1402, 1379, 1320, 1258, 1003;

${ }^{1} \mathrm{H}$ NMR $\left(500 \mathrm{MHz}, \mathrm{CDCl}_{3}\right): 6.17(1 \mathrm{H}, \mathrm{s}), 5.48(1 \mathrm{H}, \mathrm{s}), 3.36(1 \mathrm{H}, \mathrm{dd}, 13.8 \mathrm{~Hz}, 3.0 \mathrm{~Hz})$, $3.12(1 \mathrm{H}, \mathrm{dd}, 13.8 \mathrm{~Hz}, 6.2 \mathrm{~Hz}), 2.99(1 \mathrm{H}, \mathrm{d}, 5.6 \mathrm{~Hz}), 2.47(2 \mathrm{H}, \mathrm{t}, 7.4 \mathrm{~Hz}), 1.73(1 \mathrm{H}, \mathrm{OH})$, $1.60(2 \mathrm{H}, \mathrm{m}), 1.34(4 \mathrm{H}, \mathrm{m}), 0.90(3 \mathrm{H}, \mathrm{t}, 6.9 \mathrm{~Hz})$;

${ }^{13} \mathrm{C}$ NMR (125MHz, $\left.\mathrm{CDCl}_{3}\right): 153.1,134.3,75.2,60.6,31.2,27.2,27.1,22.2,13.8$;

HRMS calcd for $\mathrm{C}_{9} \mathrm{H}_{15} \mathrm{O}_{1} \mathrm{~S}_{1} 171.0844$, found $171.0836\left(\left[\mathrm{M}-\mathrm{H}_{2} \mathrm{O}\right]^{+}\right)$

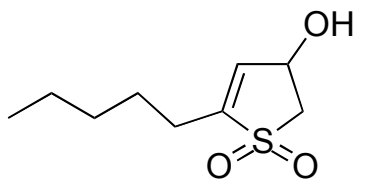

(13)

To a stirred solution of the sulfone $11(0.200 \mathrm{~g}, 0.99 \mathrm{mmol})$ in dry methanol $(10 \mathrm{ml})$ at -10 ${ }^{\circ} \mathrm{C}$, was added cerium chloride heptahydrate $(0.037 \mathrm{~g}, 0.10 \mathrm{mmol})$ in one portion. The resulting solution was stirred for 10 minutes and sodium borohydride $(0.083 \mathrm{~g}, 2.18 \mathrm{mmol})$ was added portionwise. The resulting solution was stirred for 90 minutes at $0 \sim 5{ }^{\circ} \mathrm{C}$ then solvent was removed under reduced pressure. The resulting mixture was partitioned between water and ethyl acetate and extracted with ethyl acetate $(30 \mathrm{ml} \times 3)$. The combined organic layers were washed with brine and dried over magnesium sulfate and concentrated in vacuo. The residue was purified by flash chromatography on silica gel (eluent 60/40 hexanes/ethyl acetate) to give alcohol $\mathbf{1 3}$ as a yellowish solid $(0.190 \mathrm{~g}$, $94 \%)$.

Mp: $53-54{ }^{\circ} \mathrm{C}$

$\mathrm{R}_{\mathrm{f}}=0.26(50: 50 \mathrm{EtOAc} / \mathrm{Hex})$

IR (neat) 3462, 2931, 2865, 1460, 1295, 1144, 1110, 1061;

${ }^{1} \mathrm{H}$ NMR $\left(500 \mathrm{MHz}, \mathrm{CDCl}_{3}\right): 6.28(1 \mathrm{H}, \mathrm{s}), 4.98(1 \mathrm{H}, \mathrm{s}), 3.60(1 \mathrm{H}, \mathrm{dd}, 13.8 \mathrm{~Hz}, 7.3 \mathrm{~Hz})$, $3.18(1 \mathrm{H}, \mathrm{dd}, 13.8 \mathrm{~Hz}, 3.4 \mathrm{~Hz}), 2.92(1 \mathrm{H}, \mathrm{s}), 2.40(2 \mathrm{H}, \mathrm{t}, 7.7 \mathrm{~Hz}), 1.63(2 \mathrm{H}, \mathrm{m}), 1.34(4 \mathrm{H}$, $\mathrm{m}), 0.89(3 \mathrm{H}, \mathrm{t}, 7.1 \mathrm{~Hz})$;

${ }^{13} \mathrm{C}$ NMR (125MHz, $\left.\mathrm{CDCl}_{3}\right): 147.3,133.0,65.9,57.0,31.1,26.8,23.2,22.1,13.8 ;$

HRMS calcd for $\mathrm{C}_{9} \mathrm{H}_{17} \mathrm{O}_{3} \mathrm{~S}_{1} 205.0898$, found $205.0905\left(\mathrm{M}^{+}\right)$ 


\section{A Representative Procedure for the Carbamate Formation:}

To a stirred solution of the alcohol $13(0.360 \mathrm{~g}, 1.76 \mathrm{mmol})$ in dry tetrahydrofuran $(6 \mathrm{ml})$ at ambient temperature, was added triethylamine $(0.49 \mathrm{ml}, 3.52 \mathrm{mmol})$ and benzyl isocyanate $(0.22 \mathrm{ml}, 1.76 \mathrm{mmol})$ sequentially. The resulting mixture was stirred for 24 hours and volatiles were removed under reduced pressure to give an analytically pure carbamate 14 as a thick syrup $(0.595 \mathrm{~g}, 100 \%)$.

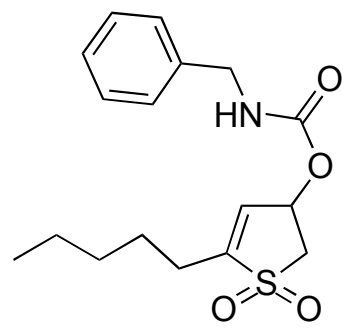

(14)

$\mathrm{R}_{\mathrm{f}}=0.69(50: 50 \mathrm{EtOAc} / \mathrm{Hex})$;

IR (neat) 3335, 2957, 2931, 2869, 1696, 1541, 1455, 1298, 1266, 1146, 1112, 1041, 985, 742, 699;

${ }^{1} \mathrm{H}$ NMR (500MHz, $\left.\mathrm{CDCl}_{3}\right): 7.33-7.24(5 \mathrm{H}, \mathrm{m}), 6.26(1 \mathrm{H}, \mathrm{s}), 5.74(1 \mathrm{H}, \mathrm{s}), 5.42(1 \mathrm{H}, \mathrm{s})$, $4.32(2 \mathrm{H}, \mathrm{d}, 5.9 \mathrm{~Hz}), 3.61(1 \mathrm{H}, \mathrm{dd}, 14.2 \mathrm{~Hz}, 7.6 \mathrm{~Hz}), 3.19(1 \mathrm{H}, \mathrm{dd}, 14.2 \mathrm{~Hz}, 1.9 \mathrm{~Hz}), 2.40$ $(2 \mathrm{H}, \mathrm{t}, 7.4 \mathrm{~Hz}), 1.62(2 \mathrm{H}, \mathrm{m}), 1.32(4 \mathrm{H}, \mathrm{m}), 0.89(3 \mathrm{H}, \mathrm{t}, 6.9 \mathrm{~Hz})$;

${ }^{13} \mathrm{C}$ NMR $\left(125 \mathrm{MHz}, \mathrm{CDCl}_{3}\right): 154.9,149.2,137.6,128.9,128.4,127.5,127.3,67.4,54.3$, 45.0, 31.0, 26.6, 23.3, 22.0, 13.7;

HRMS calcd for $\mathrm{C}_{17} \mathrm{H}_{23} \mathrm{~N}_{1} \mathrm{O}_{4} \mathrm{~S}_{1} \mathrm{Na}_{1} 360.1246$, found $360.1229\left(\mathrm{MNa}^{+}\right)$<smiles>CCCCCC1=CC(OC(=O)NCc2ccccc2)CS1=O</smiles>

cis-Sulfoxide (14)

$\mathrm{R}_{\mathrm{f}}=0.15\left(5: 95 \mathrm{MeOH} / \mathrm{CH}_{2} \mathrm{Cl}_{2}\right)$;

IR (neat); 3288, 3057, 3031, 2952, 2931, 2858, 1723, 1534, 1455, 1253, 1124, 1025, 743, 698; 
${ }^{1} \mathrm{H}$ NMR (500MHz, $\left.\mathrm{CDCl}_{3}\right): 7.34-7.25(5 \mathrm{H}, \mathrm{m}), 6.17(1 \mathrm{H}, \mathrm{s}), 6.09(1 \mathrm{H}, \mathrm{t}, 3.4 \mathrm{~Hz}), 5.14$ $(1 \mathrm{H}, \mathrm{s}), 4.34(2 \mathrm{H}, \mathrm{d}, 5.8 \mathrm{~Hz}), 3.58(1 \mathrm{H}, \mathrm{d}, 14.0 \mathrm{~Hz}), 3.12(1 \mathrm{H}, \mathrm{dd}, 14.0 \mathrm{~Hz}, 6.4 \mathrm{~Hz}), 2.48$ $(2 \mathrm{H}, \mathrm{t}, 7.2 \mathrm{~Hz}), 1.67-1.59(2 \mathrm{H}, \mathrm{m}), 1.35(4 \mathrm{H}, \mathrm{m}), 0.89(3 \mathrm{H}, \mathrm{t}, 7.1 \mathrm{~Hz})$;

${ }^{13} \mathrm{C}$ NMR $\left(125 \mathrm{MHz}, \mathrm{CDCl}_{3}\right): 156.6,155.2,137.8,129.1,128.6,127.6,127.4,77.0,58.6$, 45.1, 31.2, 27.0, 26.9, 22.2, 13.8;

HRMS calcd for $\mathrm{C}_{17} \mathrm{H}_{24} \mathrm{~N}_{1} \mathrm{O}_{3} \mathrm{~S}_{1} 322.1477$, found $322.1466\left(\mathrm{MH}^{+}\right)$

\section{$\underline{\text { A Representative Procedure for a Michael/Fragmentation Sequence: (Sulfone) }}$}

To a stirred solution of the carbamate $14(0.595 \mathrm{~g}, 1.76 \mathrm{mmol})$ in dry tetrahydrofuran $(50 \mathrm{ml})$ at $0{ }^{\circ} \mathrm{C}$, was added tert-butanol $(1.69 \mathrm{ml}, 17.6 \mathrm{mmol})$ followed by potassium tertbutoxide $(0.198 \mathrm{~g}, 1.76 \mathrm{mmol})$. The resulting solution was stirred for 5 minutes and quenched with saturated ammonium chloride solution. The mixture was extracted using ethyl acetate $(100 \mathrm{ml} \times 3)$. The combined organic layers were washed with brine and dried over magnesium sulfate and concentrated in vacuo. The residue was purified by flash chromatography on silica gel (eluent 90/10 hexanes/ethyl acetate) to give 2,3-transsulfone $16(0.354 \mathrm{~g}, 69 \%)$ and 2,3-cis-sulfone $16(0.080 \mathrm{~g}, 16 \%)$ as thick syrup. The stereochemistry of the product was assigned based upon the coupling constant of $\mathrm{H}_{2}-\mathrm{H}_{3}$ ( $7.2 \mathrm{~Hz}$ for cis- and $5.4 \mathrm{~Hz}$ for trans-isomer).

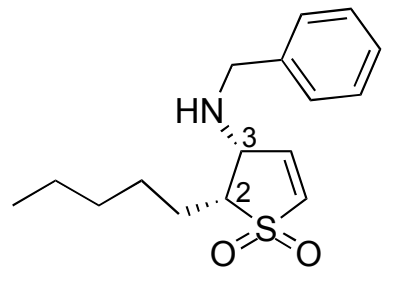

2,3-cis-Sulfone (16)

$\mathrm{R}_{\mathrm{f}}=0.41(50: 50 \mathrm{EtOAc} / \mathrm{Hex})$;

IR (neat) 3330, 3065, 2955, 2928, 2858, 1455, 1291, 1131, 1104, 735, 699, 621;

${ }^{1} \mathrm{H}$ NMR $\left(500 \mathrm{MHz}, \mathrm{CDCl}_{3}\right): 7.35-7.27(5 \mathrm{H}, \mathrm{m}), 6.88(1 \mathrm{H}, \mathrm{dd}, 7.0 \mathrm{~Hz}, 4.1 \mathrm{~Hz}), 6.63(1 \mathrm{H}$, d, $7.0 \mathrm{~Hz}), 3.92(1 \mathrm{H}, \mathrm{d}, 13.4 \mathrm{~Hz}), 3.89(1 \mathrm{H}, \mathrm{dd}, 7.2 \mathrm{~Hz}, 4.3 \mathrm{~Hz}), 3.83(1 \mathrm{H}, \mathrm{d}, 13.4 \mathrm{~Hz}), 3.11$ $(1 \mathrm{H}, \mathrm{dt}, 7.2 \mathrm{~Hz}, 7.2 \mathrm{~Hz}), 1.95(1 \mathrm{H}, \mathrm{m}), 1.85(1 \mathrm{H}, \mathrm{m}), 1.53(2 \mathrm{H}, \mathrm{m}), 1.36(4 \mathrm{H}, \mathrm{m}), 0.91(3 \mathrm{H}$, t, $7.0 \mathrm{~Hz})$;

${ }^{13} \mathrm{C}$ NMR $\left(125 \mathrm{MHz}, \mathrm{CDCl}_{3}\right): 141.3,139.4,131.8,128.5,128.0,127.4,62.1,57.1,51.4$, $31.7,26.5,22.8,22.3,13.9$;

HRMS calcd for $\mathrm{C}_{16} \mathrm{H}_{24} \mathrm{~N}_{1} \mathrm{O}_{2} \mathrm{~S}_{1} 294.1528$, found $294.1525\left(\mathrm{MH}^{+}\right)$ 


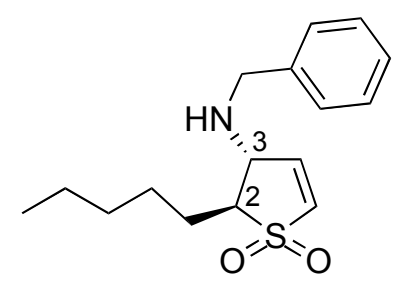

2,3-trans-Sulfone (16)

$\mathrm{R}_{\mathrm{f}}=0.33(50: 50$ EtOAc/Hex);

IR (neat) 3335, 3068, 3028, 2954, 2930, 2859, 1602, 1494, 1455, 1291, 1197, 1114, $1027,841,734,700,646$;

${ }^{1} \mathrm{H}$ NMR $\left(500 \mathrm{MHz}, \mathrm{CDCl}_{3}\right): 7.35-7.28(5 \mathrm{H}, \mathrm{m}), 6.68(1 \mathrm{H}, \mathrm{dd}, 6.9 \mathrm{~Hz}, 2.5 \mathrm{~Hz}), 6.60(1 \mathrm{H}$, dd, $6.9 \mathrm{~Hz}, 1.6 \mathrm{~Hz}), 3.89(1 \mathrm{H}, \mathrm{d}, 13.2 \mathrm{~Hz}), 3.86(1 \mathrm{H}, \mathrm{d}, 13.2 \mathrm{~Hz}), 3.71(1 \mathrm{H}, \mathrm{dt}, \mathbf{5 . 4 H z}$, 2.5Hz), $3.00(1 \mathrm{H}, \mathrm{td}, 8.29 \mathrm{~Hz}, 5.4 \mathrm{~Hz}), 1.99(1 \mathrm{H}, \mathrm{m}), 1.70(1 \mathrm{H}, \mathrm{m}), 1.63(2 \mathrm{H}, \mathrm{m}), 1.34$ $(4 \mathrm{H}, \mathrm{m}), 0.90(3 \mathrm{H}, \mathrm{t}, 7.2 \mathrm{~Hz})$;

${ }^{13} \mathrm{C}$ NMR $\left(125 \mathrm{MHz}, \mathrm{CDCl}_{3}\right): 140.5,138.9,132.1,128.6,128.0,127.5,64.4,62.6,50.8$, $31.5,27.9,26.9,22.2,13.9$;

HRMS calcd for $\mathrm{C}_{16} \mathrm{H}_{24} \mathrm{~N}_{1} \mathrm{O}_{2} \mathrm{~S}_{1} 294.1528$, found $294.1517\left(\mathrm{MH}^{+}\right)$

\section{$\underline{\text { A Representative Procedure for a Michael/Fragmentation Sequence: (Sulfoxide) }}$}

To a stirred solution of the cis-sulfoxide $12(0.120 \mathrm{~g}, 0.637 \mathrm{mmol})$ in dry tetrahydrofuran $(5 \mathrm{ml})$ at ambient temperature, was added triethylamine $(0.089 \mathrm{ml}, 0.637 \mathrm{mmol})$ and benzyl isocyanate $(0.087 \mathrm{ml}, 0.701 \mathrm{mmol})$ sequentially. The resulting mixture was stirred for 24 hours and dry tetrahydrofuran $(25 \mathrm{ml})$ was introduced to the reaction mixture. To this solution at $0{ }^{\circ} \mathrm{C}$, was added tert-butanol $(0.61 \mathrm{ml}, 6.37 \mathrm{mmol})$ followed by potassium tertbutoxide $(0.072 \mathrm{~g}, 0.637 \mathrm{mmol})$. The resulting solution was stirred for 5 minutes and quenched with saturated ammonium chloride solution. The mixture was extracted using ethyl acetate $(50 \mathrm{ml} \times 3)$. The combined organic layers were washed with saturated brine and dried over magnesium sulfate and concentrated in vacuo. The residue was purified by flash chromatography on silica gel (eluent 50/50 hexanes/ethyl acetate) to give 2,3-cissulfoxide $16(0.056 \mathrm{~g}, 32 \%)$ and 2,3-trans-sulfoxide $16(0.085 \mathrm{~g}, 49 \%)$ as thick syrup. The stereochemistry of the product was retrospectively assigned after converting $\mathbf{1 6}$ to urea 5 and 6. 


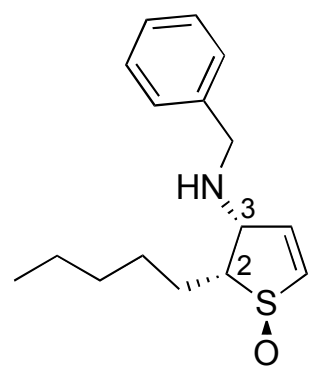

2,3-cis-Sulfoxide (16)

$\mathrm{R}_{\mathrm{f}}=0.19\left(50: 50 \mathrm{EtOAc} / \mathrm{CH}_{2} \mathrm{Cl}_{2}\right)$;

IR (neat); 3304, 3060, 3027, 2954, 2928, 2854, 1452, 1027, 737, 698;

${ }^{1} \mathrm{H}$ NMR $\left(500 \mathrm{MHz}, \mathrm{CDCl}_{3}\right): 7.34-7.24(5 \mathrm{H}, \mathrm{m}), 6.99(1 \mathrm{H}, \mathrm{dd}, 6.1 \mathrm{~Hz}, 3.1 \mathrm{~Hz}), 6.83(1 \mathrm{H}$, d, $6.1 \mathrm{~Hz}), 3.94(1 \mathrm{H}, \mathrm{d}, 13.5 \mathrm{~Hz}), 3.88(1 \mathrm{H}, \mathrm{m}), 3.84(1 \mathrm{H}, \mathrm{d}, 13.5 \mathrm{~Hz}), 2.67(1 \mathrm{H}, \mathrm{dt}, 8.4 \mathrm{~Hz}$, 6.4Hz), $1.99(1 \mathrm{H}, \mathrm{m}), 1.92(1 \mathrm{H}, \mathrm{m}), 1.50(2 \mathrm{H}, \mathrm{m}), 1.38(4 \mathrm{H}, \mathrm{m}), 0.91(3 \mathrm{H}, \mathrm{t}, 7.0 \mathrm{~Hz})$;

${ }^{13} \mathrm{C}$ NMR (125MHz, $\left.\mathrm{CDCl}_{3}\right): 146.2,140.0,134.1,128.3,128.0,127.1,64.3,63.6,51.8$, $31.8,27.4,23.0,22.4,13.9$;

HRMS calcd for $\mathrm{C}_{16} \mathrm{H}_{23} \mathrm{~N}_{1} \mathrm{O}_{1} \mathrm{~S}_{1} \mathrm{Na} 300.1398$, found $300.1383\left(\mathrm{MNa}^{+}\right)$

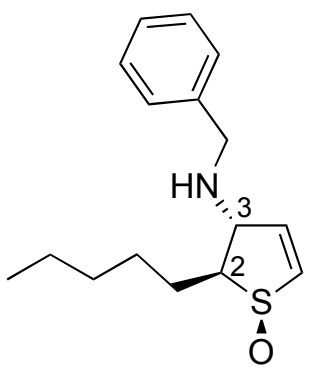

\section{2,3-trans-Sulfoxide (16)}

$\mathrm{R}_{\mathrm{f}}=0.06\left(50: 50 \mathrm{EtOAc} / \mathrm{CH}_{2} \mathrm{Cl}_{2}\right)$;

IR (neat); 3300, 3028, 2956, 2928, 2856, 1493, 1452, 1027, 732, 699;

${ }^{1} \mathrm{H}$ NMR $\left(500 \mathrm{MHz}, \mathrm{CDCl}_{3}\right): 7.34-7.25(5 \mathrm{H}, \mathrm{m}), 6.63(1 \mathrm{H}, \mathrm{dd}, 6.2 \mathrm{~Hz}, 0.9 \mathrm{~Hz}), 6.59(1 \mathrm{H}$, dd, $6.1 \mathrm{~Hz}, 2.3 \mathrm{~Hz}), 3.89(2 \mathrm{H}, \mathrm{s}), 3.59(1 \mathrm{H}, \mathrm{t}, 2.1 \mathrm{~Hz}), 3.03(1 \mathrm{H}, \mathrm{ddd}, 10.8 \mathrm{~Hz}, 6.1 \mathrm{~Hz}$, $4.8 \mathrm{~Hz}), 1.75(2 \mathrm{H}, \mathrm{m}), 1.55(2 \mathrm{H}, \mathrm{m}), 1.31(4 \mathrm{H}, \mathrm{m}), 0.89(3 \mathrm{H}, \mathrm{t}, 7.1 \mathrm{~Hz})$;

${ }^{13} \mathrm{C}$ NMR (125MHz, $\left.\mathrm{CDCl}_{3}\right): 141.7,139.3,134.2,128.4,128.0,127.2,74.7,67.4,51.3$, 31.5, 29.8, 27.5, 22.2, 13.8;

HRMS calcd for $\mathrm{C}_{16} \mathrm{H}_{24} \mathrm{~N}_{1} \mathrm{O}_{1} \mathrm{~S}_{1} 278.1579$, found $278.1573\left(\mathrm{MH}^{+}\right)$ 


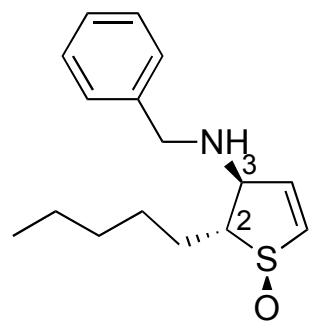

\section{2,3-trans-Sulfoxide (16a)}

2,3-trans-Sulfoxide 16a was obtained from the cis-sulfoxide 12 in 50\% yield along with 2,3-cis-sulfoxide 16a in 32\% yield.

$\mathrm{R}_{\mathrm{f}}=0.26\left(10: 90\right.$ Acetone $\left./ \mathrm{CH}_{2} \mathrm{Cl}_{2}\right)$;

IR (neat); 3288, 3029, 2954, 2927, 2855, 1494, 1453, 1139, 1026, 741, 698;

${ }^{1} \mathrm{H}$ NMR $\left(500 \mathrm{MHz}, \mathrm{CDCl}_{3}\right)$ : 7.34-7.28 $(4 \mathrm{H}, \mathrm{m}), 7.28-7.26(1 \mathrm{H}, \mathrm{m}), 6.80(1 \mathrm{H}, \mathrm{dd}, 6.2 \mathrm{~Hz}$, $1.7 \mathrm{~Hz}), 6.73(1 \mathrm{H}, \mathrm{ddd}, 7.0 \mathrm{~Hz}, 2.0 \mathrm{~Hz}, 0.8 \mathrm{~Hz}), 4.23(1 \mathrm{H}, \mathrm{d}, 5.9 \mathrm{~Hz}), 3.90(1 \mathrm{H}, \mathrm{d}, 13.1 \mathrm{~Hz})$, $3.83(1 \mathrm{H}, \mathrm{d}, 13.1 \mathrm{~Hz}), 2.57(1 \mathrm{H}, \mathrm{ddd}, 10.3 \mathrm{~Hz}, 6.6 \mathrm{~Hz}, 4.7 \mathrm{~Hz}), 2.00(1 \mathrm{H}, \mathrm{m}), 1.78(1 \mathrm{H}, \mathrm{m})$, $1.60(1 \mathrm{H}, \mathrm{m}), 1.47(1 \mathrm{H}, \mathrm{m}), 1.35(4 \mathrm{H}, \mathrm{m}), 0.90(3 \mathrm{H}, \mathrm{t}, 7.2 \mathrm{~Hz})$;

${ }^{13} \mathrm{C}$ NMR (125MHz, $\left.\mathrm{CDCl}_{3}\right): 146.5,139.4,133.9,128.5,128.0,127.4,68.7,65.1,51.9$, 31.6, 28.2, 24.7, 22.4, 13.9;

HRMS calcd for $\mathrm{C}_{16} \mathrm{H}_{24} \mathrm{~N}_{1} \mathrm{O}_{1} \mathrm{~S}_{1} 278.1579$, found $278.1574\left(\mathrm{MH}^{+}\right)$

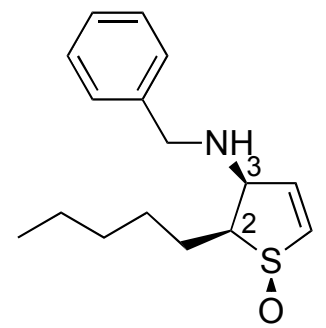

\section{2,3-cis-Sulfoxide (16a)}

$\mathrm{R}_{\mathrm{f}}=0.25\left(10: 90\right.$ Acetone $\left./ \mathrm{CH}_{2} \mathrm{Cl}_{2}\right)$;

IR (neat); 3288, 3031, 2955, 2926, 2856, 1459, 1452, 1026, 734, 698;

${ }^{1} \mathrm{H}$ NMR (500MHz, $\left.\mathrm{CDCl}_{3}\right): 7.34-7.25(5 \mathrm{H}, \mathrm{m}), 6.66(2 \mathrm{H}, \mathrm{d}, 6.3 \mathrm{~Hz}), 4.43(1 \mathrm{H}, 6.2 \mathrm{~Hz})$, $3.7(2 \mathrm{H}, \mathrm{s}), 3.16(1 \mathrm{H}, \mathrm{dt}, 8.7 \mathrm{~Hz}, 6.6 \mathrm{~Hz}), 1.88(1 \mathrm{H}, \mathrm{m}), 1.77(1 \mathrm{H}, \mathrm{m}), 1.53(2 \mathrm{H}, \mathrm{m}), 1.35$ $(4 \mathrm{H}, \mathrm{m}), 0.90(3 \mathrm{H}, \mathrm{t}, 6.9 \mathrm{~Hz})$;

${ }^{13} \mathrm{C}$ NMR (125MHz, $\left.\mathrm{CDCl}_{3}\right): 142.6,139.3,136.1,128.5,128.0,127.4,72.7,63.4,52.0$, 31.7, 27.9, 24.7, 22.3, 13.9; 
HRMS calcd for $\mathrm{C}_{16} \mathrm{H}_{24} \mathrm{~N}_{1} \mathrm{O}_{1} \mathrm{~S}_{1} 278.1579$, found $278.1577\left(\mathrm{MH}^{+}\right)$

\section{A Representative Procedure for the Urea Formation:}

To a stirred solution of the 2,3-cis-Sulfone $16(0.125 \mathrm{~g}, 0.43 \mathrm{mmol})$ in dry tetrahydrofuran $(4 \mathrm{ml})$ at ambient temperature, was added triethylamine $(0.060 \mathrm{ml}, 0.43 \mathrm{mmol})$ and benzyl isocyanate $(0.053 \mathrm{ml}, 0.43 \mathrm{mmol})$ sequentially. The resulting mixture was stirred for 24 hours and volatiles were removed under reduced pressure to give an analytically pure urea 17 as a white powder $(0.182 \mathrm{~g}, 100 \%)$.

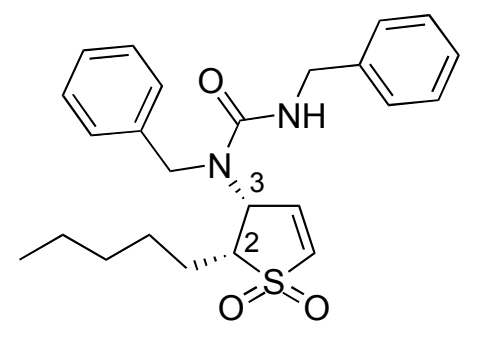

2,3-cis-Sulfone (17)

Mp: $164-165^{\circ} \mathrm{C}$

$\mathrm{R}_{\mathrm{f}}=0.31(50: 50 \mathrm{EtOAc} / \mathrm{Hex})$;

IR (neat) 3363, 3064, 3030, 2954, 2927, 2860, 1639, 1529, 1453, 1391, 1293, 1136, 1094, 912, 730, 697;

${ }^{1} \mathrm{H}$ NMR $\left(500 \mathrm{MHz}, \mathrm{CDCl}_{3}\right): 7.33(2 \mathrm{H}, \mathrm{dd}, 8.3 \mathrm{~Hz}, 6.2 \mathrm{~Hz}), 7.28-7.23(4 \mathrm{H}, \mathrm{m}), 7.16(2 \mathrm{H}$, d, $7.1 \mathrm{~Hz}), 7.07(2 \mathrm{H}, \mathrm{dd}, 8.0 \mathrm{~Hz}, 1.7 \mathrm{~Hz}), 6.70(1 \mathrm{H}, \mathrm{dd}, 6.9 \mathrm{~Hz}, 1.6 \mathrm{~Hz}), 6.55(6.9 \mathrm{~Hz}, 4.2 \mathrm{~Hz})$, $6.15(1 \mathrm{H}, \mathrm{s}), 4.87(1 \mathrm{H}, \mathrm{t}, 5.5 \mathrm{~Hz}), 4.43-4.31(4 \mathrm{H}, \mathrm{m}), 3.35(1 \mathrm{H}, \mathrm{ddd}, 12.1 \mathrm{~Hz}, 8.2 \mathrm{~Hz}$, $3.9 \mathrm{~Hz}), 1.80(1 \mathrm{H}, \mathrm{m}), 1.69(2 \mathrm{H}, \mathrm{m}), 1.43(1 \mathrm{H}, \mathrm{m}), 1.35(4 \mathrm{H}, \mathrm{m}), 0.90(3 \mathrm{H}, \mathrm{t}, 7.0 \mathrm{~Hz})$;

${ }^{13} \mathrm{C}$ NMR $\left(125 \mathrm{MHz}, \mathrm{CDCl}_{3}\right): 157.8,138.7,137.6,136.4,135.7,129.1,128.5,127.8$, $127.2,125.7,61.1,56.2,47.5,44.9,31.7,26.8,23.7,22.2,13.9$;

HRMS calcd for $\mathrm{C}_{24} \mathrm{H}_{30} \mathrm{~N}_{2} \mathrm{O}_{3} \mathrm{~S}_{1} \mathrm{Na}_{1} 449.1875$, found $449.1881\left(\mathrm{MNa}^{+}\right)$

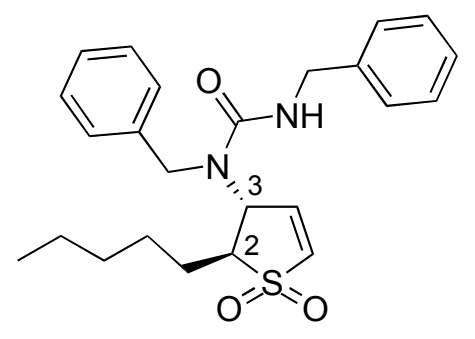

2,3-trans-Sulfone (17) 
$\mathrm{R}_{\mathrm{f}}=0.17(50: 50 \mathrm{EtOAc} / \mathrm{Hex})$;

IR (neat) 3364, 3063, 3031, 2929, 2860, 1636, 1533, 1453, 1391, 1362, 1295, 1131, 1098, 730, 698;

${ }^{1} \mathrm{H}$ NMR $\left(500 \mathrm{MHz}, \mathrm{CDCl}_{3}\right): 7.34(2 \mathrm{H}, \mathrm{dd}, 8.0 \mathrm{~Hz}, 1.3 \mathrm{~Hz}), 7.30-7.20(4 \mathrm{H}, \mathrm{m}), 7.18(2 \mathrm{H}$, d, 7.4Hz), $7.05(2 \mathrm{H}, \mathrm{d}, 7.4 \mathrm{~Hz}), 6.56(1 \mathrm{H}, \mathrm{dd}, 6.7 \mathrm{~Hz}, 2.1 \mathrm{~Hz}), 6.48(1 \mathrm{H}, \mathrm{dd}, 6.9 \mathrm{~Hz}, 2.2 \mathrm{~Hz})$, $5.59(1 \mathrm{H}, \mathrm{s}), 5.01(1 \mathrm{H}, \mathrm{s}), 4.33(4 \mathrm{H}, \mathrm{m}), 3.69(1 \mathrm{H}, \mathrm{t}, 5.3 \mathrm{~Hz}), 2.99(1 \mathrm{H}, \mathrm{dt}, 9.2 \mathrm{~Hz}, 5.2 \mathrm{~Hz})$, $2.00(1 \mathrm{H}, \mathrm{m}), 1.87(1 \mathrm{H}, \mathrm{m}), 1.82(1 \mathrm{H}, \mathrm{m}), 1.60(1 \mathrm{H}, \mathrm{m}), 1.44(1 \mathrm{H}, \mathrm{m}), 1.30(4 \mathrm{H}, \mathrm{m}), 0.89$ $(3 \mathrm{H}, \mathrm{t}, 6.9 \mathrm{~Hz})$;

${ }^{13} \mathrm{C}$ NMR $\left(125 \mathrm{MHz}, \mathrm{CDCl}_{3}\right): 157.3,139.8,138.5,136.1,133.7,129.1,128.3,127.9$, 127.0, 125.7, 62.3, 60.5, 47.1, 44.7, 31.3, 27.4, 26.5, 22.0, 13.7;

HRMS calcd for $\mathrm{C}_{24} \mathrm{H}_{30} \mathrm{~N}_{2} \mathrm{O}_{3} \mathrm{~S}_{1} \mathrm{Na}_{1} 449.1875$, found $449.1888\left(\mathrm{MNa}^{+}\right)$

\section{A Representative Procedure for the Bicyclic Urea Formation through A Tandem} Michael reaction sequence:

To a stirred solution of the $\mathbf{1 2}$ or $\mathbf{1 3}(0.637 \mathrm{mmol})$ in dry tetrahydrofuran $(5 \mathrm{ml})$ at ambient temperature, was added triethylamine $(0.637 \mathrm{mmol})$ and benzyl isocyanate $(0.701 \mathrm{mmol})$ sequentially. The resulting mixture was stirred for 24 hours and dry tetrahydrofuran $(25 \mathrm{ml})$ was introduced to the reaction mixture. To this solution at $0{ }^{\circ} \mathrm{C}$, was added tertbutanol $(0.61 \mathrm{ml}, 6.37 \mathrm{mmol})$ followed by potassium tert-butoxide $(0.072 \mathrm{~g}, 0.637 \mathrm{mmol})$. The resulting solution was stirred for 2 minutes and benzyl isocyanate $(1.75 \mathrm{mmol})$ was introduced. The reaction mixture was stirred for 1 hour while monitored by tlc. The reaction was quenched with saturated sodium carbonate and solvent was removed under reduced pressure. The resulting oily residue was purified by flash chromatography on silica gel (eluent $80 / 20$ hexanes/ethyl acetate) to give the corresponding urea $\mathbf{4}$ and $\mathbf{6}$ as a white solid (55-80\%).

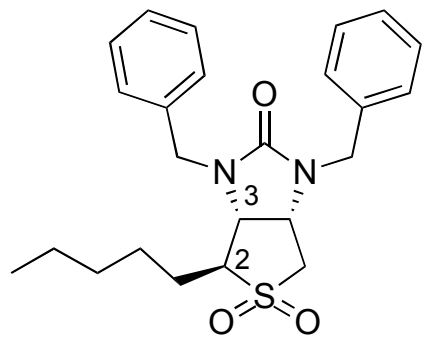

2,3-trans-Sulfone (4)

Preparation using potassium tert-butoxide in tetrahydrofuran and tert-butanol

To a stirred solution of the 2,3-cis-sulfone 17 or the 2,3-trans-sulfone $17(0.100 \mathrm{~g}$, $0.23 \mathrm{mmol})$ in dry tetrahydrofuran $(10 \mathrm{ml})$ at $0{ }^{\circ} \mathrm{C}$, was added tert-butanol $(0.23 \mathrm{ml}$, $2.34 \mathrm{mmol})$ followed by potassium tert-butoxide $(0.026 \mathrm{~g}, 0.23 \mathrm{mmol})$. The resulting 
solution was stirred for 5 minutes and quenched with saturated ammonium chloride solution. The mixture was extracted using ethyl acetate $(20 \mathrm{ml} \times 3)$. The combined organic layers were washed with brine and dried over magnesium sulfate and concentrated in vacuo. The residue was purified by flash chromatography on silica gel (eluent 80/20 hexanes/ethyl acetate) to give 2,3-trans-sulfone urea 4 as a white solid $(0.090 \mathrm{~g}, 90 \%)$.

\section{Preparation using sodium hydride in tetrahydrofuran}

To a stirred solution of the 2,3-cis-sulfone 17 or the 2,3-trans-sulfone $17(0.100 \mathrm{~g}$, $0.23 \mathrm{mmol})$ in dry tetrahydrofuran $(10 \mathrm{ml})$ at $0{ }^{\circ} \mathrm{C}$, was added sodium hydride $(0.009 \mathrm{~g}$, $0.23 \mathrm{mmol}, 60 \% \mathrm{w} / \mathrm{w}$ ) in one portion. The resulting mixture was stirred for one hour. The reaction was quenched using one drop of water and solvent was removed under reduced pressure. The resulting oily residue was purified by flash chromatography on silica gel (eluent 80/20 hexanes/ethyl acetate) to give 2,3-trans-sulfone urea 4 as a white solid (0.099g, 99\%).

Preparation using 1,8-diazabicyclo[5.4.0]undec-7-ene in tetrahydrofuran

To a stirred solution of the 2,3-cis-sulfone 17 or the 2,3-trans-sulfone $17(0.100 \mathrm{~g}$, $0.23 \mathrm{mmol})$ in dry tetrahydrofuran $(10 \mathrm{ml})$ at $0{ }^{\circ} \mathrm{C}$, was added 1,8 diazabicyclo[5.4.0] undec-7-ene $(0.035 \mathrm{ml}, 0.02 \mathrm{mmol})$. The resulting mixture was stirred for 24 hour while monitoring by ${ }^{1} \mathrm{H}$ NMR. The reaction solvent was removed under reduced pressure and the resulting oily residue was purified by flash chromatography on silica gel (eluent 80/20 hexanes/ethyl acetate) to give 2,3-trans-sulfone urea 4 as a white solid $(0.099 \mathrm{~g}, 99 \%)$.

Mp: $108-109{ }^{\circ} \mathrm{C}$

$\mathrm{R}_{\mathrm{f}}=0.52(50: 50 \mathrm{EtOAc} / \mathrm{Hex})$

IR (neat) 2928, 2860, 1703, 1449, 1359, 1312, 1242, 1130, 748, 702;

${ }^{1} \mathrm{H}$ NMR (500MHz, $\left.\mathrm{CDCl}_{3}\right): 7.37-7.23(10 \mathrm{H}, \mathrm{m}), 4.71(1 \mathrm{H}, \mathrm{d}, 15.6 \mathrm{~Hz}), 4.69(1 \mathrm{H}, \mathrm{d}$, $14.9 \mathrm{~Hz}), 4.34(1 \mathrm{H}, \mathrm{d}, 15.6 \mathrm{~Hz}), 4.25(1 \mathrm{H}, \mathrm{d}, 14.9 \mathrm{~Hz}), 4.05(1 \mathrm{H}, \mathrm{dt}, 9.5 \mathrm{~Hz}, 7.5 \mathrm{~Hz}), 3.73$

$(1 \mathrm{H}, \mathrm{dd}, 9.5 \mathrm{~Hz}, 5.5 \mathrm{~Hz}), 3.11(1 \mathrm{H}, \mathrm{dd}, 13.6 \mathrm{~Hz}, 7.1 \mathrm{~Hz}), 3.04(1 \mathrm{H}, \mathrm{dt}, 9.3 \mathrm{~Hz}, 5.0 \mathrm{~Hz}), 2.96$

$(1 \mathrm{H}, \mathrm{dd}, 13.5 \mathrm{~Hz}, 7.7 \mathrm{~Hz}), 1.85(1 \mathrm{H}, \mathrm{m}), 1.43(2 \mathrm{H}, \mathrm{m}), 1.23(2 \mathrm{H}, \mathrm{m}), 1.15(2 \mathrm{H}, \mathrm{m}), 0.84$ $(3 \mathrm{H}, \mathrm{t}, 7.0 \mathrm{~Hz})$;

${ }^{13} \mathrm{C}$ NMR $\left(125 \mathrm{MHz}, \mathrm{CDCl}_{3}\right): 158.9,136.2,135.9,129.0,128.9,128.3,128.2,127.9$, 127.7, 63.6, 59.3, 53.0, 51.2, 47.2, 47.1, 31.3, 27.4, 26.3, 22.1, 13.8;

HRMS calcd for $\mathrm{C}_{24} \mathrm{H}_{30} \mathrm{~N}_{2} \mathrm{O}_{3} \mathrm{~S}_{1} 426.1977$, found $426.1963\left(\mathrm{M}^{+}\right)$ 


\section{The Stereochemical Assignment of Sulfone (4)}

We have assigned the stereochemistry of the sulfone (4) with direct comparison with the known biotin sulfone derivatives $(\mathbf{A}$ and $\mathbf{B}){ }^{2}$ We noted different chemical shifts for sulfone (4) from those of sulfone (A and $\mathbf{B}$ ). A distinguished chemical shift pattern for $\mathrm{H}_{5 \text { endo/sexo }}$ as well as $\mathrm{C}_{5}$ of (4) is noteworthy.

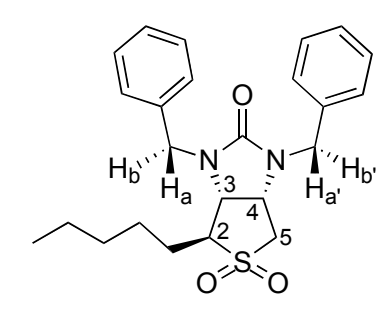

2,3-trans-Sulfone (4)

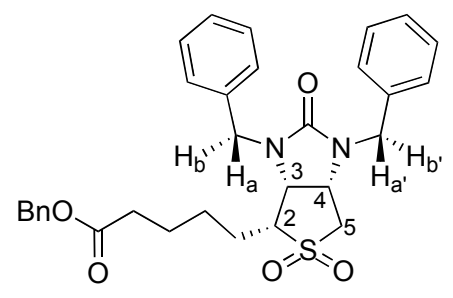

(A)

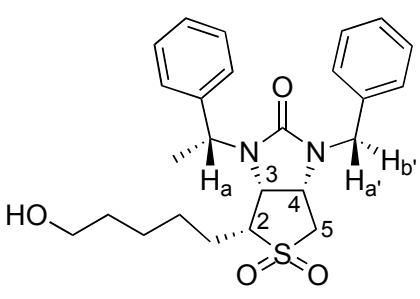

(B)

Table 1. ${ }^{1} \mathbf{H}$ NMR Chemical Shifts of Sulfones with a $\mathbf{C}_{2}$ substituent $(500 \mathrm{MHz}$; $\left.\mathrm{CDCl}_{3}\right)$

\begin{tabular}{|c|c|c|c|}
\hline & $\mathbf{4}$ & $\mathbf{A}^{2}$ & $\mathbf{B}^{2}$ \\
\hline $\mathrm{H}_{2}$ & 3.03 & 2.82 & 2.86 \\
\hline $\mathrm{H}_{3}$ & 3.73 & 4.01 & 4.02 \\
\hline $\mathrm{H}_{4}$ & 4.06 & 4.17 & 4.18 \\
\hline $\mathbf{H}_{\text {5endo/5exo }}$ & $\mathbf{3 . 1 2 / 2 . 9 6}$ & $\mathbf{2 . 9 8 / 2 . 9 8}$ & $\mathbf{2 . 9 9 / 2 . 9 8}$ \\
\hline $\mathrm{H}_{\mathrm{a}}$ & 4.25 & 4.03 & 4.04 \\
\hline $\mathrm{H}_{\mathrm{a}^{\prime}}$ & 4.34 & 4.18 & 4.19 \\
\hline $\mathrm{H}_{\mathrm{b}}$ & 4.71 & 4.88 & 4.91 \\
\hline $\mathrm{H}_{\mathrm{b}}$, & 4.69 & 4.64 & 4.64 \\
\hline
\end{tabular}

Table 2. ${ }^{13} \mathbf{C}$ NMR Chemical Shifts of Sulfones with a $\mathbf{C}_{2}$ substituent $(125 \mathrm{MHz}$; $\left.\mathrm{CDCl}_{3}\right)$

\begin{tabular}{|c|c|c|c|}
\hline & $\mathbf{4}$ & $\mathbf{A}^{2}$ & $\mathbf{B}^{2}$ \\
\hline $\mathrm{Bn}-\mathrm{C}$ & $47.15 / 47.25$ & $47.44 / 47.99$ & $47.59 / 48.04$ \\
\hline $\mathrm{C}_{2}$ & 63.60 & 61.32 & 61.54 \\
\hline $\mathrm{C}_{3} / \mathrm{C}_{4}$ & $51.25 / 59.38$ & $51.91 / 55.20$ & $52.01 / 55.27$ \\
\hline $\mathbf{C}_{5}$ & $\mathbf{5 3 . 0 0}$ & $\mathbf{5 0 . 5 0}$ & $\mathbf{5 0 . 6 6}$ \\
\hline
\end{tabular}

${ }^{2}$ Oh, K. Tetrahedron Lett. 2007, 48, 3685. 


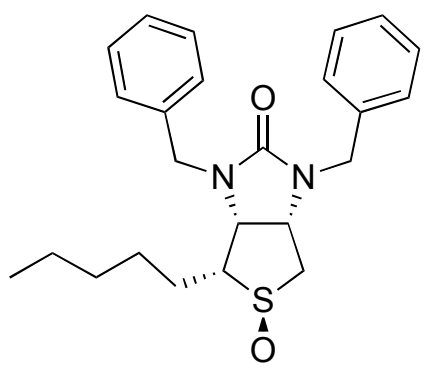

2,3-cis-Sulfoxide (6a)

To a stirred solution of the 2,3-cis-sulfoxide $12(0.050 \mathrm{~g}, 0.180 \mathrm{mmol})$ in dry tetrahydrofuran $(3 \mathrm{ml})$ at ambient temperature, was added triethylamine $(0.025 \mathrm{ml}$, $0.180 \mathrm{mmol})$ and benzyl isocyanate $(0.023 \mathrm{ml}, 0.180 \mathrm{mmol})$ sequentially. The resulting mixture was stirred for 24 hours and dry tetrahydrofuran $(7 \mathrm{ml})$ was introduced to the reaction mixture. To this solution at $0{ }^{\circ} \mathrm{C}$, was added tert-butanol $(0.17 \mathrm{ml}, 1.80 \mathrm{mmol})$ followed by potassium tert-butoxide $(0.020 \mathrm{~g}, 0.180 \mathrm{mmol})$. The resulting solution was stirred for 5 minutes and quenched with saturated sodium carbonate solution. The mixture was extracted using ethyl acetate $(10 \mathrm{ml} \times 3)$. The combined organic layers were washed with brine and dried over magnesium sulfate and concentrated in vacuo. The residue was purified by flash chromatography on silica gel (eluent 10/90 acetone/dichloromethane) to give 2,3-cis-sulfoxide urea 6a $(0.068 \mathrm{~g}, 92 \%)$ as a thick syrup.

$\mathrm{R}_{\mathrm{f}}=0.18\left(10: 90\right.$ Acetone $\left./ \mathrm{CH}_{2} \mathrm{Cl}_{2}\right)$;

IR (neat); 2926, 2856, 1688, 1449, 1356, 1239, 1040;

${ }^{1} \mathrm{H}$ NMR (500MHz, $\left.\mathrm{CDCl}_{3}\right): 7.35-7.22(10 \mathrm{H}, \mathrm{m}), 5.22(1 \mathrm{H}, \mathrm{d}, 15.5 \mathrm{~Hz}), 4.99(1 \mathrm{H}, \mathrm{d}$, $15.4 \mathrm{~Hz}), 4.23(1 \mathrm{H}, \mathrm{ddd}, 8.5 \mathrm{~Hz}, 6.7 \mathrm{~Hz}, 1.5 \mathrm{~Hz}), 4.13(1 \mathrm{H}, \mathrm{d}, 15.3 \mathrm{~Hz}), 4.12(1 \mathrm{H}, \mathrm{dd}, 8.7 \mathrm{~Hz}$, $6.2 \mathrm{~Hz}), 4.05(1 \mathrm{H}, \mathrm{d}, 15.5 \mathrm{~Hz}), 3.36(1 \mathrm{H}, \mathrm{d}, 14.8 \mathrm{~Hz}), 2.56(1 \mathrm{H}, \mathrm{m}), 2.48(1 \mathrm{H}, \mathrm{dd}, 14.8 \mathrm{~Hz}$, 6.7Hz), $2.31(1 \mathrm{H}, \mathrm{m}), 1.87(1 \mathrm{H}, \mathrm{m}), 1.71(1 \mathrm{H}, \mathrm{m}), 1.43-1.34(5 \mathrm{H}, \mathrm{m}), 0.91(3 \mathrm{H}, \mathrm{t}, 7.0 \mathrm{~Hz})$;

${ }^{13} \mathrm{C}$ NMR $\left(125 \mathrm{MHz}, \mathrm{CDCl}_{3}\right): 159.4,136.7,136.3,128.7,128.7,128.2,128.2,127.7$, 127.6, 68.0, 60.7, 59.5, 51.8, 48.6, 46.3, 31.8, 28.7, 23.7, 22.4, 13.9;

HRMS calcd for $\mathrm{C}_{24} \mathrm{H}_{31} \mathrm{~N}_{2} \mathrm{O}_{2} \mathrm{~S}_{1} 411.2106$, found $411.2102\left(\mathrm{MH}^{+}\right)$

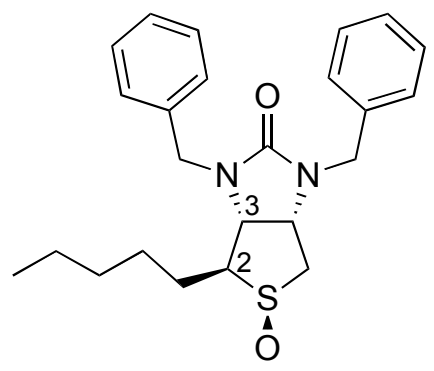

2,3-trans-Sulfoxide (6) 
$\mathrm{R}_{\mathrm{f}}=0.09\left(10: 90\right.$ Acetone $\left./ \mathrm{CH}_{2} \mathrm{Cl}_{2}\right)$;

IR (neat); 3062, 3028, 2956, 2928, 2858, 1694, 1495, 1464, 1449, 1358, 1241, 1044, 746, 700 ;

${ }^{1} \mathrm{H}$ NMR $\left(500 \mathrm{MHz}, \mathrm{CDCl}_{3}\right)$ : 7.35-7.26 $(10 \mathrm{H}, \mathrm{m}), 4.96(1 \mathrm{H}, \mathrm{d}, 15.7 \mathrm{~Hz}), 4.92(1 \mathrm{H}, \mathrm{d}$, $15.6 \mathrm{~Hz}), 4.27(1 \mathrm{H}, \mathrm{ddd}, 8.6 \mathrm{~Hz}, 6.6 \mathrm{~Hz}, 2.8 \mathrm{~Hz}), 4.20(1 \mathrm{H}, \mathrm{d}, 15.4 \mathrm{~Hz}), 4.18(1 \mathrm{H}, \mathrm{d}$, $15.3 \mathrm{~Hz}), 3.92(1 \mathrm{H}, \mathrm{dd}, 8.6 \mathrm{~Hz}, 2.0 \mathrm{~Hz}), 3.38(1 \mathrm{H}, \mathrm{m}), 3.24(1 \mathrm{H}, \mathrm{d}, 14.5 \mathrm{~Hz}), 2.75(1 \mathrm{H}, \mathrm{dd}$, $14.5 \mathrm{~Hz}, 6.6 \mathrm{~Hz}), 1.25-1.13(7 \mathrm{H}, \mathrm{m}), 1.05(1 \mathrm{H}, \mathrm{m}), 0.82(3 \mathrm{H}, \mathrm{t}, 6.9 \mathrm{~Hz})$;

${ }^{13} \mathrm{C}$ NMR $\left(125 \mathrm{MHz}, \mathrm{CDCl}_{3}\right): 157.8,136.5,128.7,128.7,128.2,128.1,127.6,69.3,64.6$, 59.1, 53.1, 46.6, 46.3, 31.1, 28.9, 27.0, 22.1, 13.7;

HRMS calcd for $\mathrm{C}_{24} \mathrm{H}_{30} \mathrm{~N}_{2} \mathrm{O}_{2} \mathrm{~S}_{1} \mathrm{Na} 433.1926$, found $433.1910\left(\mathrm{MNa}^{+}\right)$

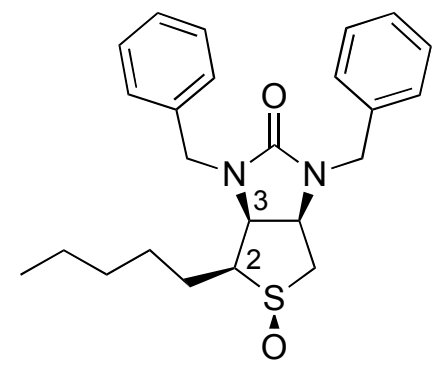

2,3-cis-Sulfoxide (5a)

$\mathrm{R}_{\mathrm{f}}=0.51\left(10: 90\right.$ Acetone $\left./ \mathrm{CH}_{2} \mathrm{Cl}_{2}\right)$;

IR (neat) 2926, 2856, 1701, 1448, 1356, 1236, 1035, 743, 702;

${ }^{1} \mathrm{H}$ NMR $\left(500 \mathrm{MHz}, \mathrm{CDCl}_{3}\right): 7.34-7.25(10 \mathrm{H}, \mathrm{m}), 4.90(1 \mathrm{H}, \mathrm{d}, 14.8 \mathrm{~Hz}), 4.62(1 \mathrm{H}, \mathrm{dd}$, $9.8 \mathrm{~Hz}, 6.3 \mathrm{~Hz}), 4.56(1 \mathrm{H}, \mathrm{d}, 14.9 \mathrm{~Hz}), 4.39(1 \mathrm{H}, \mathrm{ddd}, 9.8 \mathrm{~Hz}, 7.1 \mathrm{~Hz}, 5.9 \mathrm{~Hz}), 4.31(1 \mathrm{H}, \mathrm{d}$, $14.9 \mathrm{~Hz}), 4.07(1 \mathrm{H}, \mathrm{d}, 14.8 \mathrm{~Hz}), 3.27-3.23(1 \mathrm{H}, \mathrm{m}), 3.05(1 \mathrm{H}, \mathrm{ddd}, 14.4 \mathrm{~Hz}, 7.1 \mathrm{~Hz}, 1.5 \mathrm{~Hz})$, $2.64(1 \mathrm{H}, \mathrm{dd}, 14.4 \mathrm{~Hz}, 5.8 \mathrm{~Hz}), 1.46-1.36(2 \mathrm{H}, \mathrm{m}), 1.29-1.16(6 \mathrm{H}, \mathrm{m}), 0.88(3 \mathrm{H}, \mathrm{t}, 6.9 \mathrm{~Hz})$;

${ }^{13} \mathrm{C}$ NMR $\left(125 \mathrm{MHz}, \mathrm{CDCl}_{3}\right): 159.6,136.3,136.2,128.9,128.6,128.4,128.4,128.0$, 128.0, 68.1, 61.2, 59.0, 53.9, 47.7, 47.7, 31.4, 28.0, 24.7, 22.2, 13.8;

HRMS calcd for $\mathrm{C}_{24} \mathrm{H}_{30} \mathrm{~N}_{2} \mathrm{O}_{2} \mathrm{~S}_{1} \mathrm{Na} 433.1926$, found $433.1932\left(\mathrm{MNa}^{+}\right)$ 


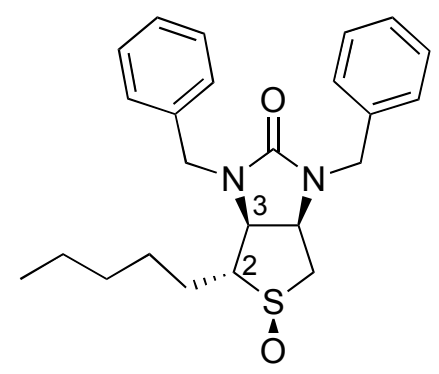

2,3-trans-Sulfoxide (5)

$\mathrm{R}_{\mathrm{f}}=0.52\left(10: 90\right.$ Acetone $\left./ \mathrm{CH}_{2} \mathrm{Cl}_{2}\right)$;

IR (neat) 2955, 2927, 1696, 1448, 1358, 1242, 1076, 1030, 749, 703;

${ }^{1} \mathrm{H}$ NMR (500MHz, $\left.\mathrm{CDCl}_{3}\right): 7.35-7.24(10 \mathrm{H}, \mathrm{m}), 4.68(1 \mathrm{H}, \mathrm{d}, 14.9 \mathrm{~Hz}), 4.64(1 \mathrm{H}, \mathrm{d}$, $15.6 \mathrm{~Hz}), 4.55(1 \mathrm{H}, \mathrm{ddd}, 9.7 \mathrm{~Hz}, 8.7 \mathrm{~Hz}, 6.3 \mathrm{~Hz}), 4.43(1 \mathrm{H}, \mathrm{d}, 15.6 \mathrm{~Hz}, 4.29(1 \mathrm{H}, \mathrm{d}, 14.9 \mathrm{~Hz})$, $3.99(1 \mathrm{H}, \mathrm{dd}, 9.7 \mathrm{~Hz}, 6.5 \mathrm{~Hz}), 3.25(1 \mathrm{H}, \mathrm{ddd}, 13.5 \mathrm{~Hz}, 6.4 \mathrm{~Hz}, 0.4 \mathrm{~Hz}), 2.72(1 \mathrm{H}, \mathrm{ddd}$, $10.8 \mathrm{~Hz}, 6.3 \mathrm{~Hz}, 3.9 \mathrm{~Hz}), 2.44(1 \mathrm{H}, \mathrm{dd}, 13.7 \mathrm{~Hz}, 8.5 \mathrm{~Hz}), 1.90-1.84(1 \mathrm{H}, \mathrm{m}), 1.60-1.55(1 \mathrm{H}$, $\mathrm{m}), 1.44-1.41(1 \mathrm{H}, \mathrm{m}), 1.27-1.12(5 \mathrm{H}, \mathrm{m}), 0.85(3 \mathrm{H}, \mathrm{t}, 7.3 \mathrm{~Hz})$;

${ }^{13} \mathrm{C}$ NMR $\left(125 \mathrm{MHz}, \mathrm{CDCl}_{3}\right): 158.4,136.9,136.5,128.8,128.8,128.5,128.3,128.0$, $127.7,127.6,69.4,63.9,59.2,54.8,47.3,47.0,31.3,27.7,26.3,22.3,13.8$;

HRMS calcd for $\mathrm{C}_{24} \mathrm{H}_{30} \mathrm{~N}_{2} \mathrm{O}_{2} \mathrm{~S}_{1} \mathrm{Na} 433.1926$, found $433.1922\left(\mathrm{MNa}^{+}\right)$

\section{The Stereochemical Assignment of Sulfoxides (5, 5a, 6, and 6a)}

We have assigned the stereochemistry of the sulfoxide (5a and 6a) with direct comparison with the known biotin sulfoxide derivatives $(\mathbf{C}, \mathbf{D}$, and $\mathbf{E}){ }^{2}$ We noted a similar chemical shift for the $\mathrm{C}_{5}$ of the sulfoxide (6a) with that of sulfoxide (C) as well as similar chemical shift patterns for $\mathrm{H}_{2}$ and $\mathrm{H}_{3}$.

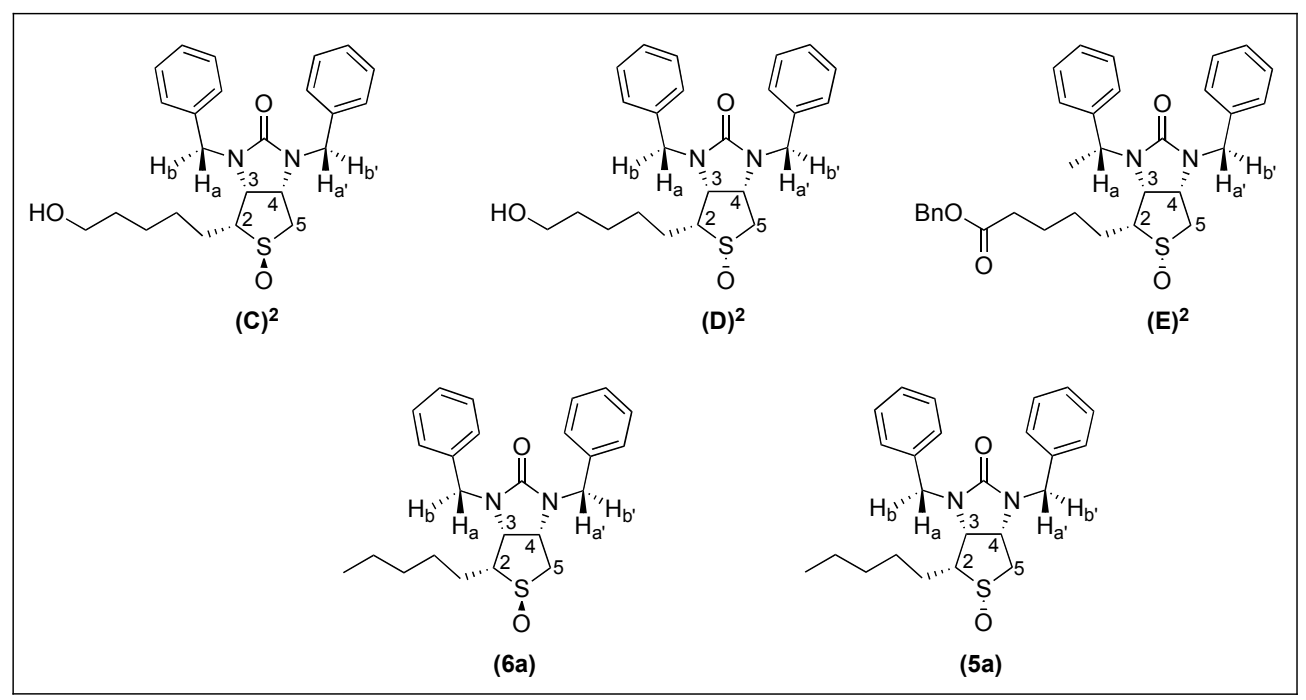


Table 3. ${ }^{1} \mathrm{H}$ NMR Chemical Shifts of Sulfoxides $\left(500 \mathrm{MHz} ; \mathrm{CDCl}_{3}\right)$

\begin{tabular}{|c|c|c|c|c|c|}
\hline & $\mathbf{C}^{\mathrm{a}}$ & $\mathbf{D}^{\mathrm{b}}$ & $\mathbf{E}^{\mathrm{b}}$ & $\mathbf{6 a}$ & $\mathbf{5 a}$ \\
\hline $\mathbf{H}_{\mathbf{2}}$ & $\mathbf{2 . 8 7}$ & $\mathbf{3 . 2 6}$ & $\mathbf{3 . 2 0}$ & $\mathbf{2 . 5 6}$ & $\mathbf{3 . 2 4}$ \\
\hline $\mathbf{H}_{\mathbf{3}}$ & $\mathbf{4 . 1 8}$ & $\mathbf{4 . 6 0}$ & $\mathbf{4 . 6 1}$ & $\mathbf{4 . 1 2}$ & $\mathbf{4 . 6 2}$ \\
\hline $\mathrm{H}_{4}$ & 4.04 & 4.37 & 4.38 & 4.22 & 4.38 \\
\hline $\mathrm{H}_{5 \text { endo/5exo }}$ & $3.00 / 3.00$ & $3.05 / 2.66$ & $3.03 / 2.62$ & $3.36 / 2.46$ & $3.04 / 2.64$ \\
\hline $\mathrm{H}_{\mathrm{a}}$ & 4.04 & 4.07 & 4.10 & 4.05 & 4.06 \\
\hline $\mathrm{H}_{\mathrm{a}^{\prime}}$ & 4.18 & 4.28 & 4.28 & 4.12 & 4.31 \\
\hline $\mathrm{H}_{\mathrm{b}}$ & 4.90 & 4.87 & 4.83 & 5.20 & 4.90 \\
\hline $\mathrm{H}_{\mathrm{b}^{\prime}}$ & 4.64 & 4.55 & 4.56 & 4.99 & 4.56 \\
\hline
\end{tabular}

Table 4. ${ }^{13} \mathrm{C}$ NMR Chemical Shifts of Sulfoxides (125 MHz; $\mathrm{CDCl}_{3}$ )

\begin{tabular}{|c|c|c|c|c|c|}
\hline & $\mathbf{C}^{\mathrm{a}}$ & $\mathbf{D}^{\mathrm{b}}$ & $\mathbf{E}^{\mathrm{b}}$ & $\mathbf{6 a}$ & $\mathbf{5 a}$ \\
\hline $\mathrm{Bn}-\mathrm{C}$ & $47.57 / 48.04$ & $47.59 / 47.75$ & $47.60 / 47.80$ & $46.30 / 48.60$ & $47.70 / 47.76$ \\
\hline $\mathbf{C}_{2}$ & $\mathbf{5 0 . 6 3}$ & $\mathbf{5 3 . 9 0}$ & $\mathbf{5 3 . 8 9}$ & $\mathbf{5 1 . 8 3}$ & $\mathbf{5 3 . 9 7}$ \\
\hline $\mathrm{C}_{3} / \mathrm{C}_{4}$ & $55.27 / 61.53$ & $61.10 / 68.06$ & $61.21 / 67.94$ & $60.71 / 68.04$ & $61.20 / 68.16$ \\
\hline $\mathrm{C}_{5}$ & 52.01 & 58.91 & 58.94 & 59.53 & 59.08 \\
\hline
\end{tabular}

The stereochemistry at the $\mathrm{C}_{2}$ substituent of sulfoxides has been assigned after deoxygenation and comparing to the known sulfides (2 and 18). ${ }^{3}$
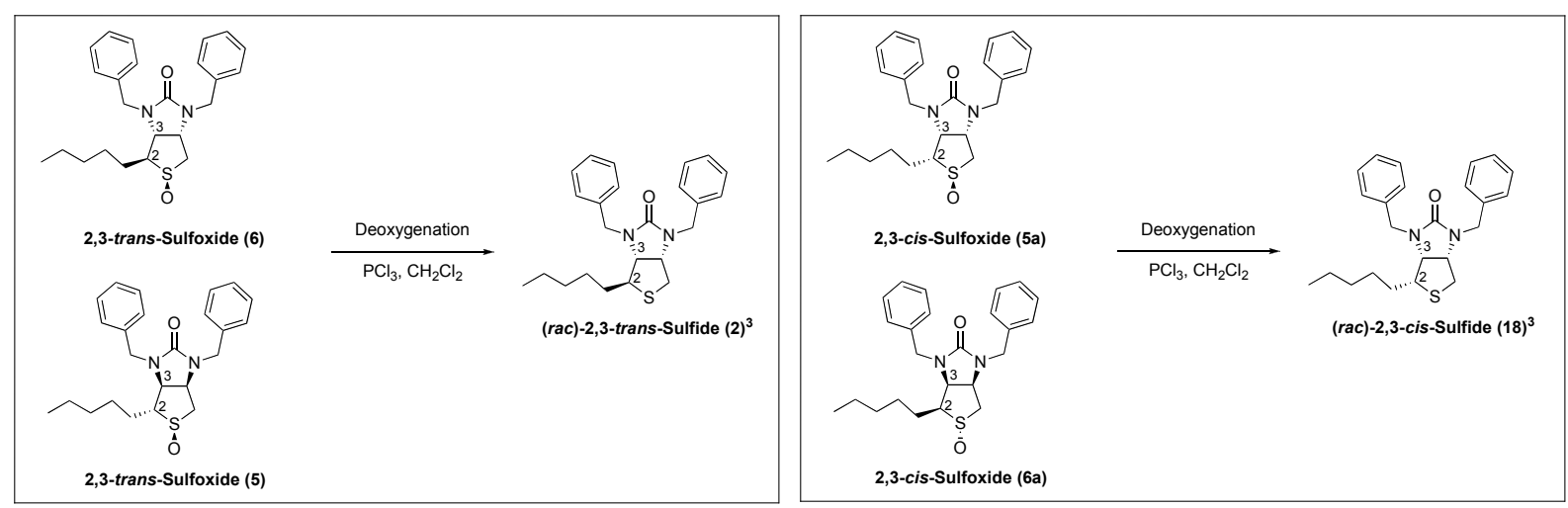

${ }^{3}$ Bates, H.; Rosenblum S. J. Org. Chem. 1986, 51, 3447. 


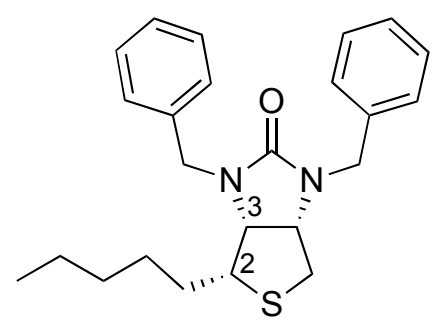

2,3-cis-Sulfide $(18)^{3}$

To a stirred solution of 2,3-cis-sulfoxide 5a $(0.033 \mathrm{~g}, 0.08 \mathrm{mmol})$ in dry dichloromethane $(3 \mathrm{ml})$ at $0{ }^{\circ} \mathrm{C}$, was added phosphorus trichloride $(0.041 \mathrm{ml}, 0.47 \mathrm{mml})$ dropwise. The resulting mixture was stirred for 60 minutes at same temperature and solvent was removed under reduced pressure. The residue in ethyl acetate $(2 \mathrm{ml})$ was hydrogenated under hydrogen atmosphere using $\mathrm{Pd} / \mathrm{C}$ for 48 hours. The catalyst was filtered and the solvent was removed under reduced pressure. The residue was purified by flash chromatography on silica gel (eluent 1/99 diethyl ether/dichloromethane) to give an $N, N^{\prime}-$ dibenzyl deoxybiotin $\mathbf{1 8}^{3}(0.032 \mathrm{~g}, 100 \%)$.

$\mathrm{R}_{\mathrm{f}}=0.50(50: 50 \mathrm{EtOAc} / \mathrm{Hex})$;

IR (neat) 3026, 2926, 2855, 1695, 1495, 1448, 1356, 1237, 701;

${ }^{1} \mathrm{H}$ NMR $\left(500 \mathrm{MHz}, \mathrm{CDCl}_{3}\right): 7.34-7.23(10 \mathrm{H}, \mathrm{m}), 5.10(1 \mathrm{H}, \mathrm{d}, 15.2 \mathrm{~Hz}), 4.75(1 \mathrm{H}$, $15.2 \mathrm{~Hz}), 4.15(1 \mathrm{H}, 15.2 \mathrm{~Hz}), 3.96(1 \mathrm{H}, \mathrm{ddd}, 9.8 \mathrm{~Hz}, 6.2 \mathrm{~Hz}, 3.9 \mathrm{~Hz}), 3.95(1 \mathrm{H}, \mathrm{d}, 15.2 \mathrm{~Hz})$, $3.85(1 \mathrm{H}, \mathrm{dd}, 9.4 \mathrm{~Hz}, 5.6 \mathrm{~Hz}), 3.08(1 \mathrm{H}, \mathrm{m}), 2.75(1 \mathrm{H}, \mathrm{dd}, 12.5 \mathrm{~Hz}, 3.9 \mathrm{~Hz}), 2.67(1 \mathrm{H}, \mathrm{dd}$, $12.5 \mathrm{~Hz}, 6.2 \mathrm{~Hz}), 1.60(1 \mathrm{H}, \mathrm{m}), 1.55-1.40(2 \mathrm{H}, \mathrm{m}), 1.31-1.25(5 \mathrm{H}, \mathrm{m}), 0.90(3 \mathrm{H}, \mathrm{t}, 6.9 \mathrm{~Hz})$;

${ }^{13} \mathrm{C}$ NMR $\left(125 \mathrm{MHz}, \mathrm{CDCl}_{3}\right): 161.0,137.0,136.9,128.7,128.6,128.2,128.2,127.6$, $127.5,62.5,61.2,54.7,47.9,46.5,34.8,31.6,28.8,28.7,22.5,14.0$;

HRMS calcd for $\mathrm{C}_{24} \mathrm{H}_{31} \mathrm{~N}_{2} \mathrm{O}_{1} \mathrm{~S}_{1}$ 395.2157, found $395.2171\left(\mathrm{MH}^{+}\right)$

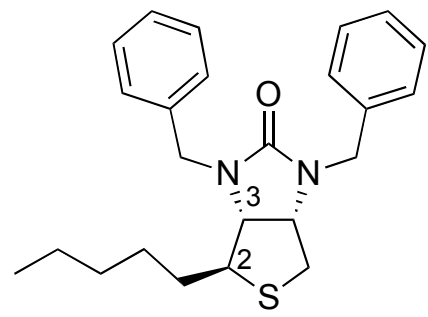

2,3-trans-Sulfide (2) ${ }^{3}$

$\mathrm{R}_{\mathrm{f}}=0.53(50: 50 \mathrm{EtOAc} / \mathrm{Hex})$

IR (neat) 2928, 2858, 1697, 1450, 1358, 1301, 1243, 1113, 747, 701; 
${ }^{1} \mathrm{H}$ NMR (500MHz, $\left.\mathrm{CDCl}_{3}\right): 7.35-7.26(10 \mathrm{H}, \mathrm{m}), 4.81(1 \mathrm{H}, \mathrm{d}, 15.2 \mathrm{~Hz}), 4.80(1 \mathrm{H}, \mathrm{d}$, $15.4 \mathrm{~Hz}), 4.21(1 \mathrm{H}, \mathrm{d}, 15.4 \mathrm{~Hz}), 4.16(1 \mathrm{H}, \mathrm{d}, 15.2 \mathrm{~Hz}), 4.03(1 \mathrm{H}, \mathrm{dt}, 8.8 \mathrm{~Hz}, 4.3 \mathrm{~Hz}), 3.66$ $(1 \mathrm{H}, \mathrm{dd}, 8.6 \mathrm{~Hz}, 2.3 \mathrm{~Hz}), 3.14(1 \mathrm{H}, \mathrm{m}), 2.83(1 \mathrm{H}, \mathrm{dd}, 12.5 \mathrm{~Hz}, 5.6 \mathrm{~Hz}), 2.73(1 \mathrm{H}, \mathrm{dd}$, $12.5 \mathrm{~Hz}, 3.6 \mathrm{~Hz}), 1.43(1 \mathrm{H}, \mathrm{m}), 1.30-1.14(7 \mathrm{H}, \mathrm{m}), 0.84(3 \mathrm{H}, \mathrm{t}, 7.0 \mathrm{~Hz})$;

${ }^{13} \mathrm{C}$ NMR $\left(125 \mathrm{MHz}, \mathrm{CDCl}_{3}\right): 159.5,137.2,137.1,128.6,128.6,128.1,128.0,127.5$, $127.5,66.6,61.3,53.7,46.7,46.7,35.4,34.2,31.2,27.9,22.4,13.9$;

HRMS calcd for $\mathrm{C}_{24} \mathrm{H}_{31} \mathrm{~N}_{2} \mathrm{O}_{1} \mathrm{~S}_{1} 395.2157$, found $395.2150\left(\mathrm{MH}^{+}\right)$ 


\section{${ }^{1}$ H NMR Spectrum of (Z)-8}

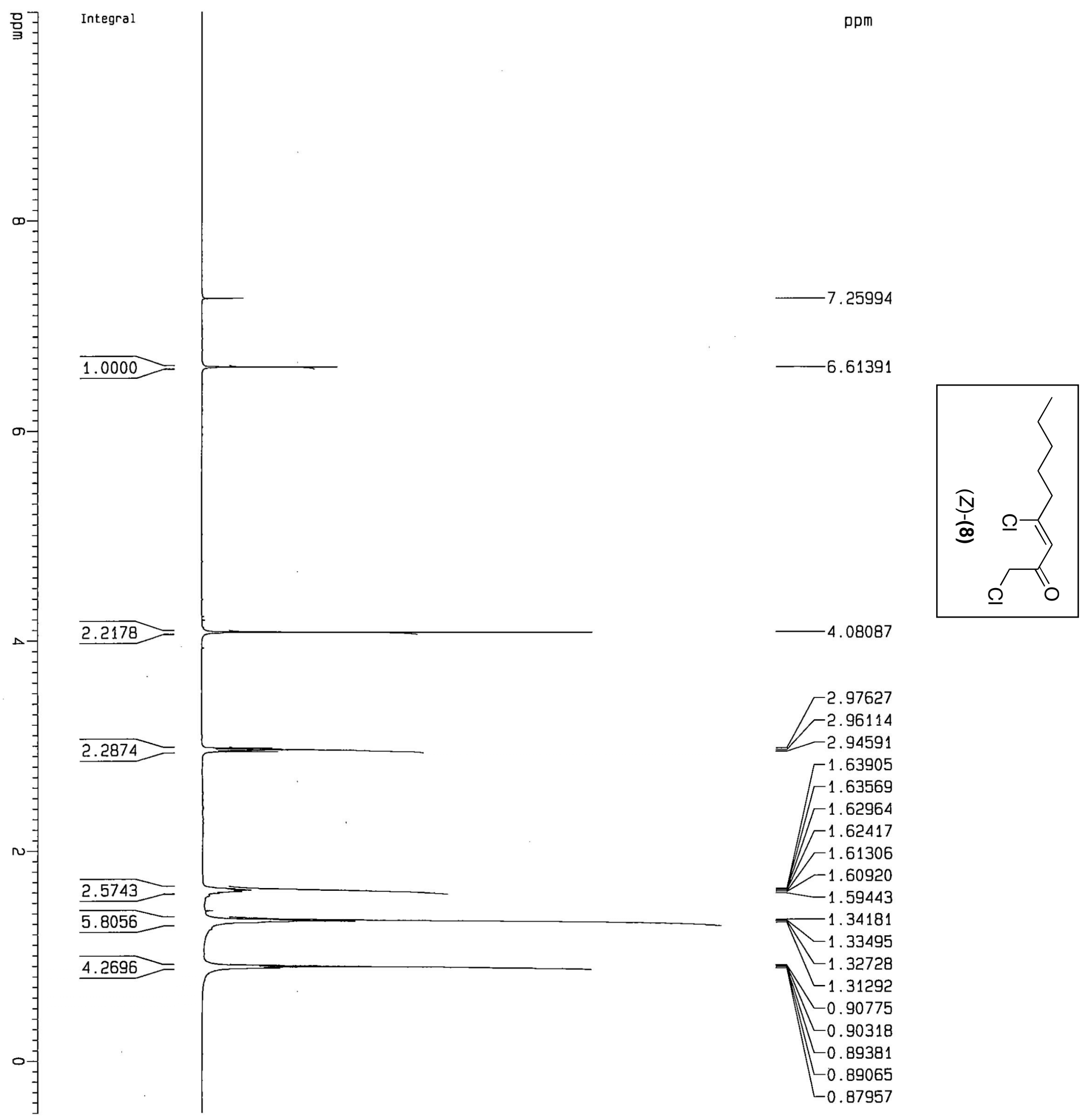




\section{${ }^{13} \mathrm{C}$ NMR Spectrum of (Z)-8}
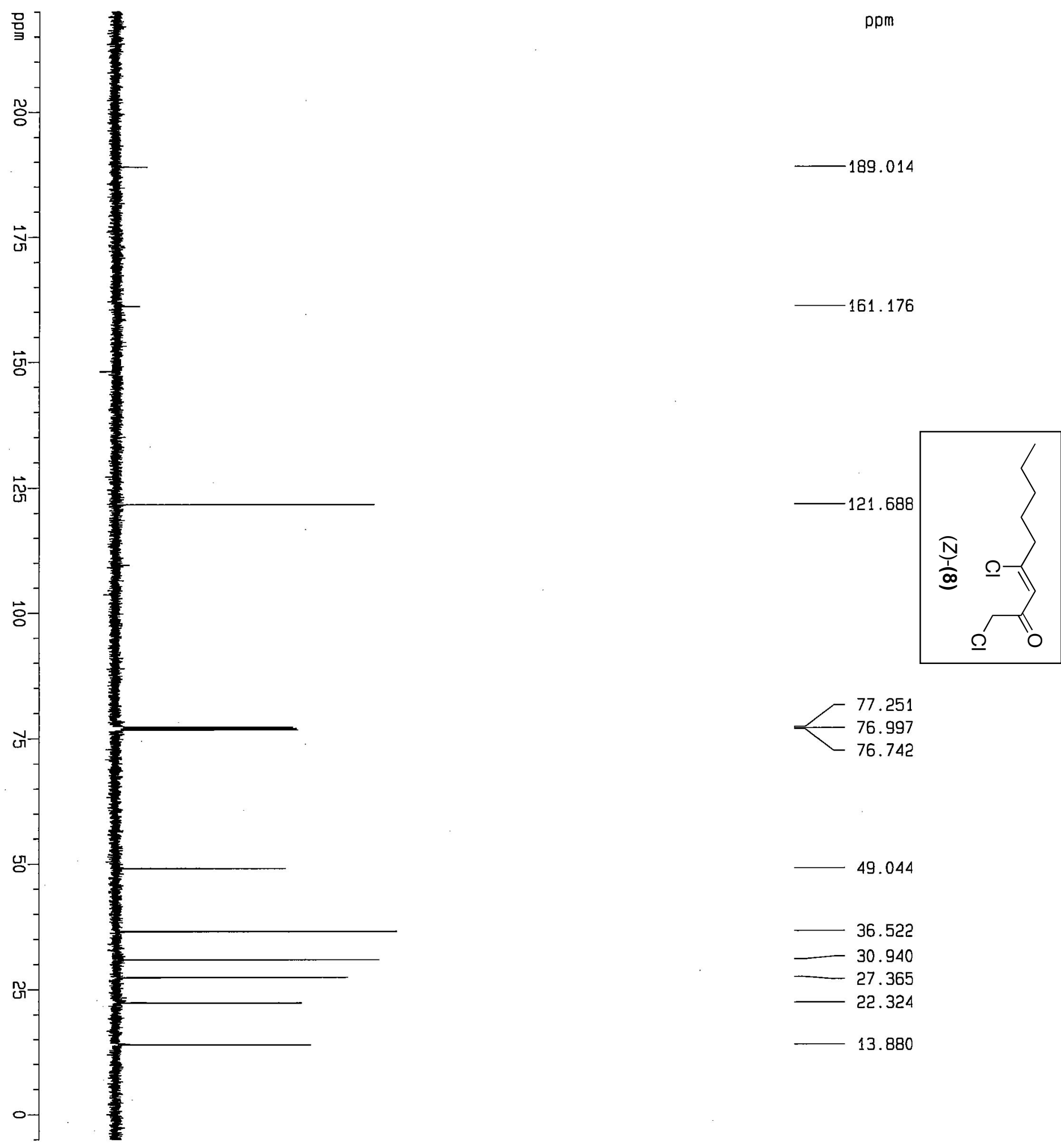

77.251

76.997

76.742

49.044

- 36.522

30.940

27.365

22.324

13.880 


\section{${ }^{1}$ H NMR Spectrum of $(E)-8$}

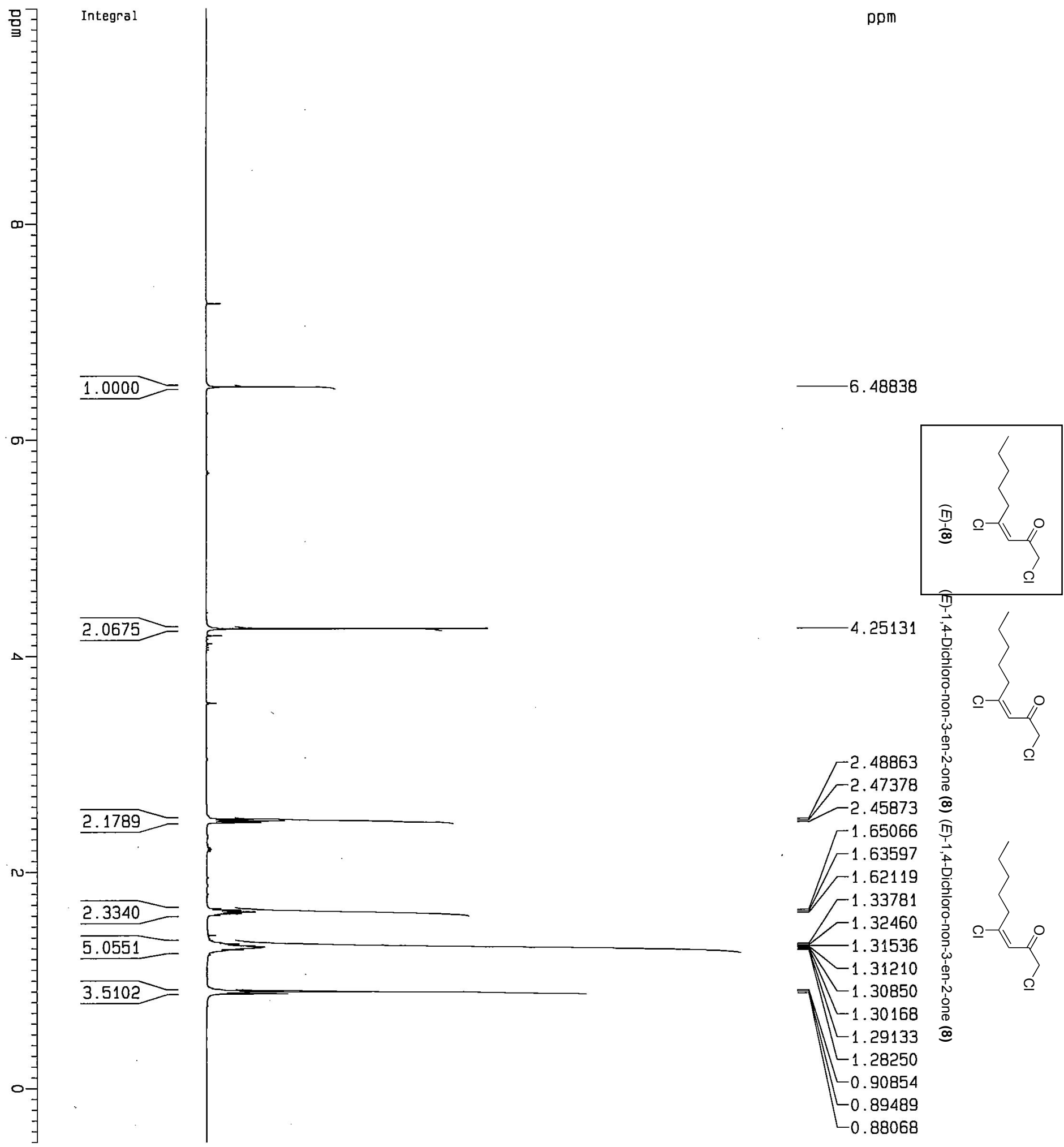




\section{${ }^{13}$ C NMR Spectrum of $(E)-8$}
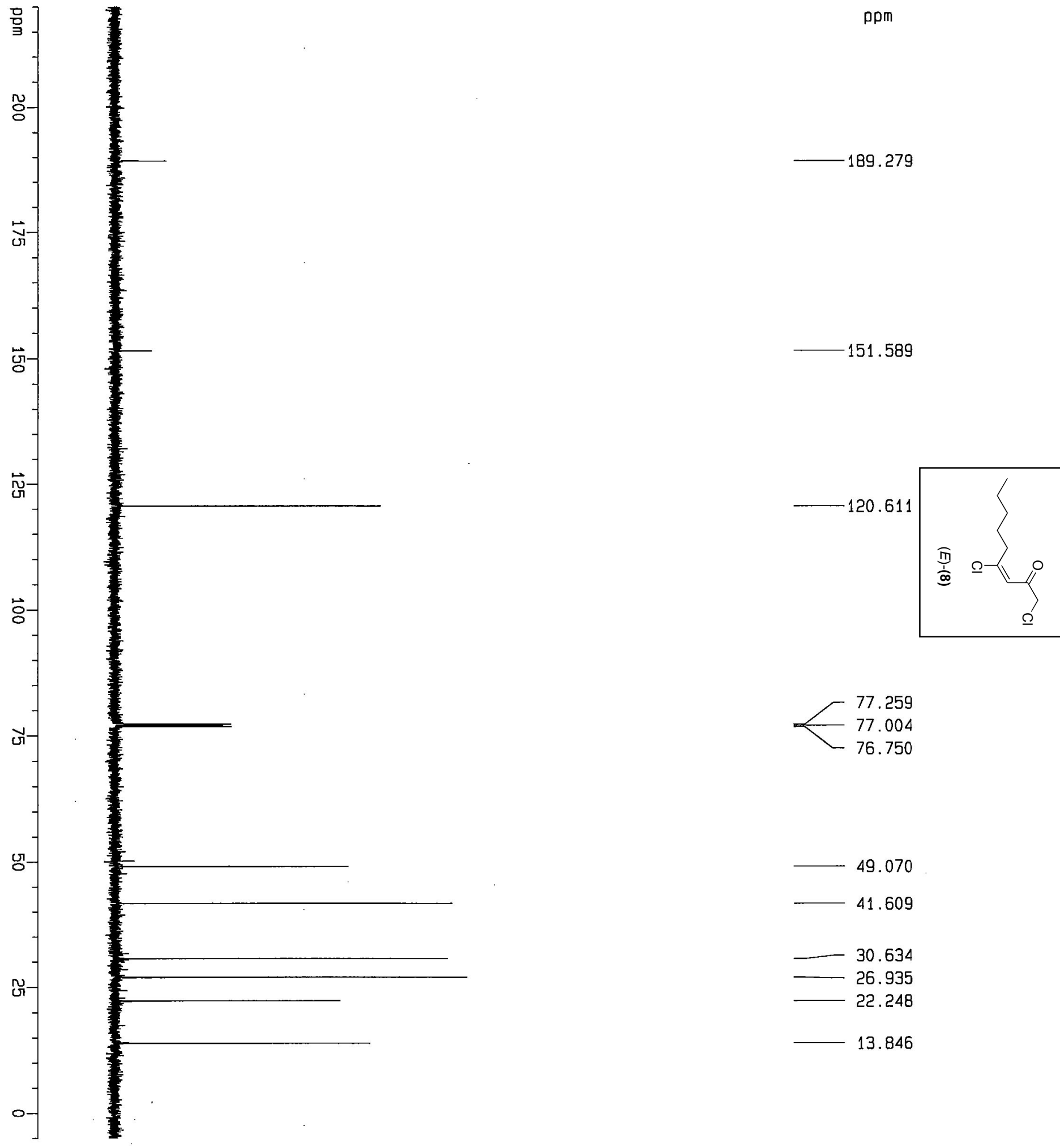


\section{${ }^{1} \mathrm{H}$ NMR Spectrum of 9}

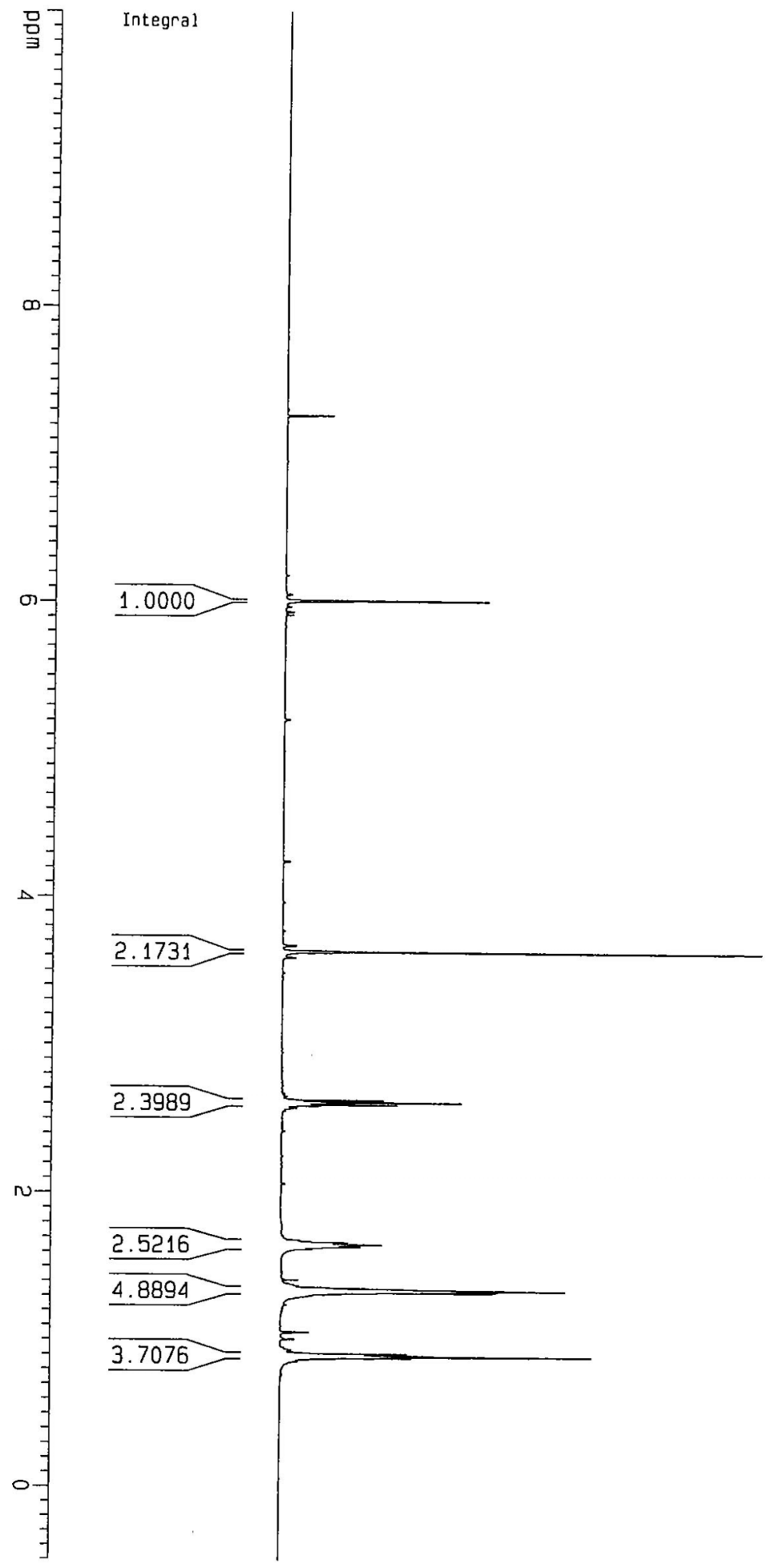

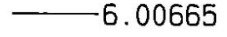

$-3.62733$

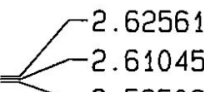

$-2.59509$

. $\quad 1.66561$

1.65101

$-1.63627$

$-1.35583$

1. 34809

$-1.34230$

$-1.33476$

$-1.32801$

$-0.90778$

$-0.89465$

$-0.88037$ 


\section{${ }^{13}$ C NMR Spectrum of 9}

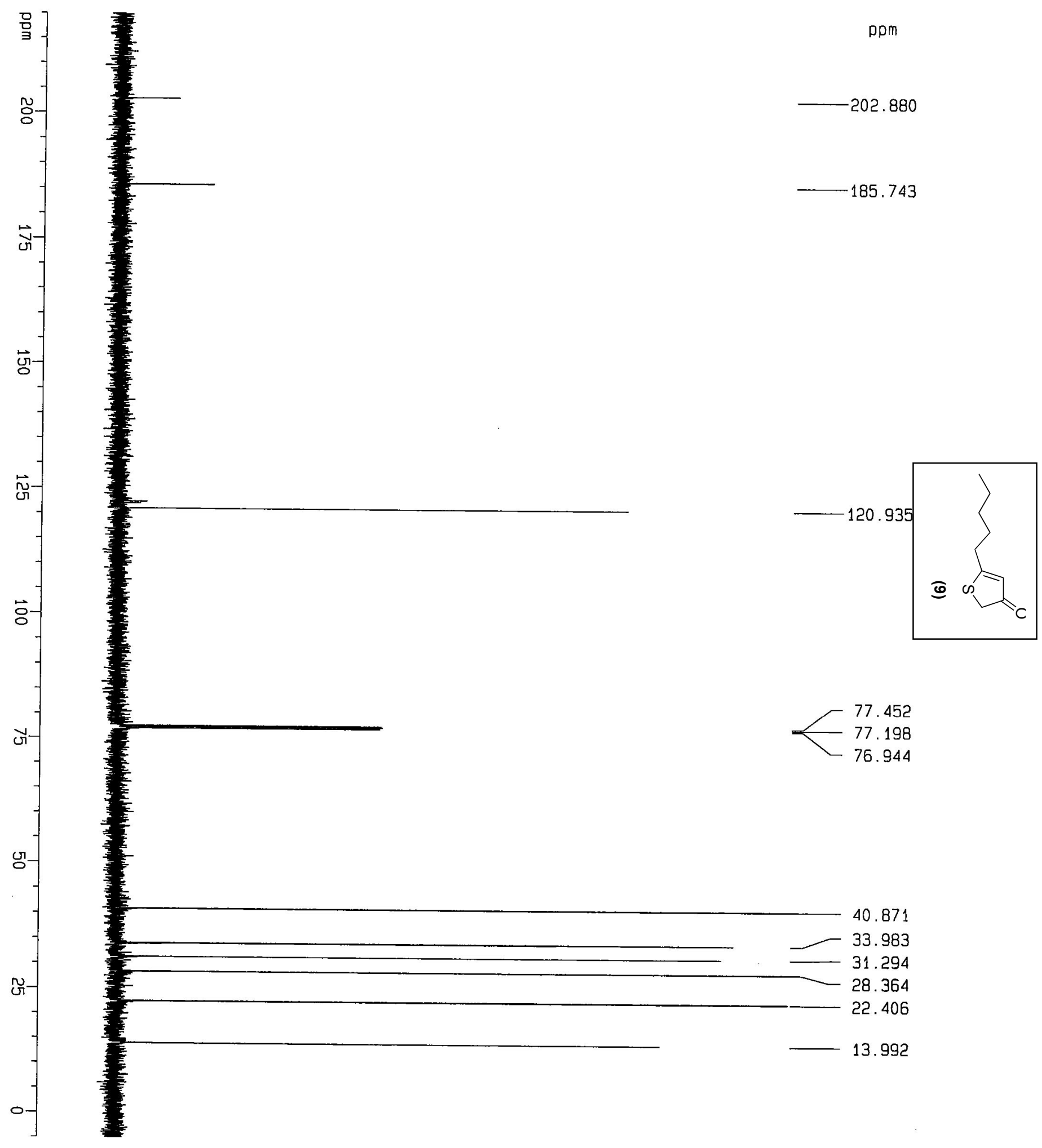




\section{${ }^{1} \mathrm{H}$ NMR Spectrum of 10}

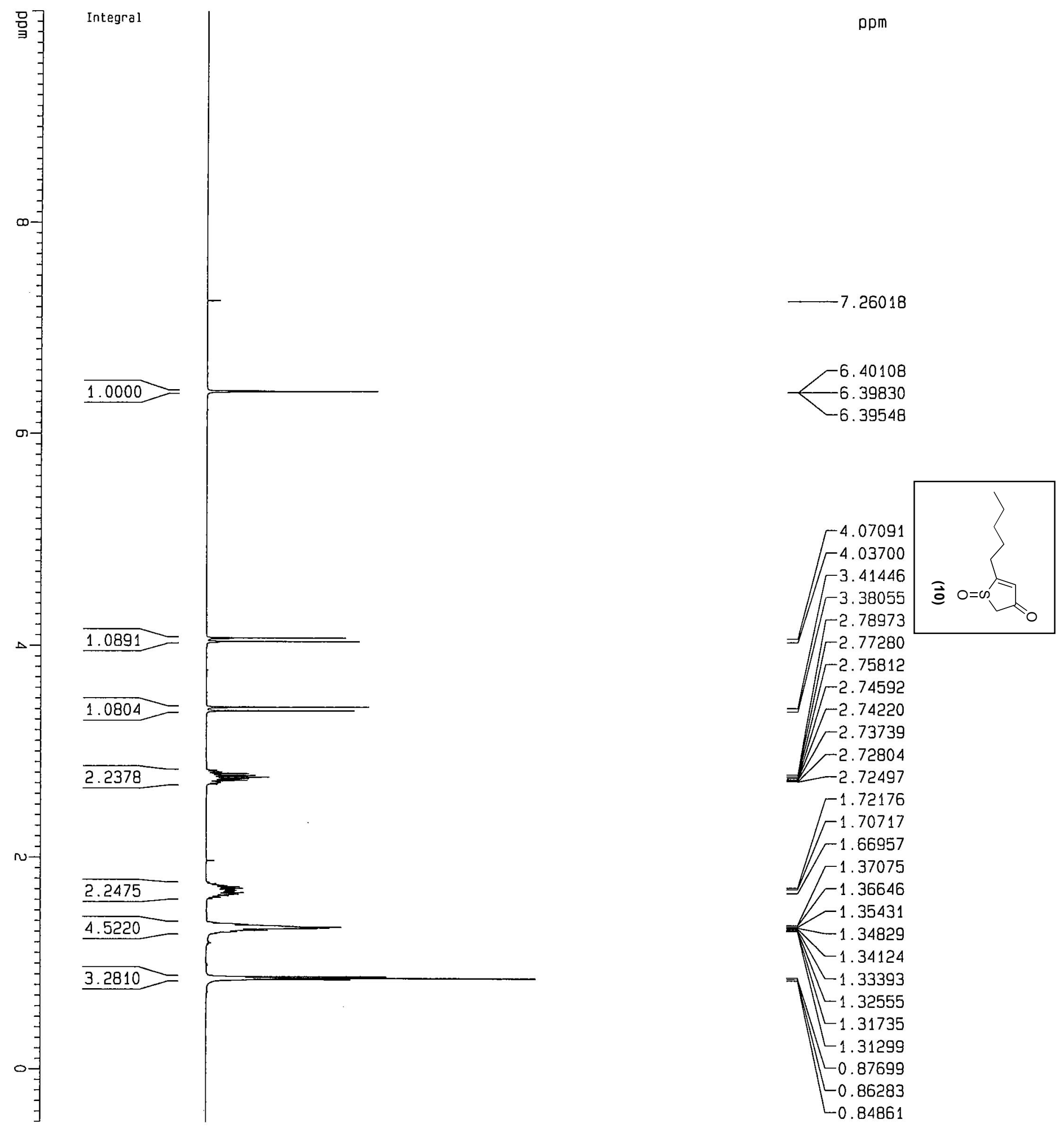




\section{${ }^{13}$ C NMR Spectrum of 10}
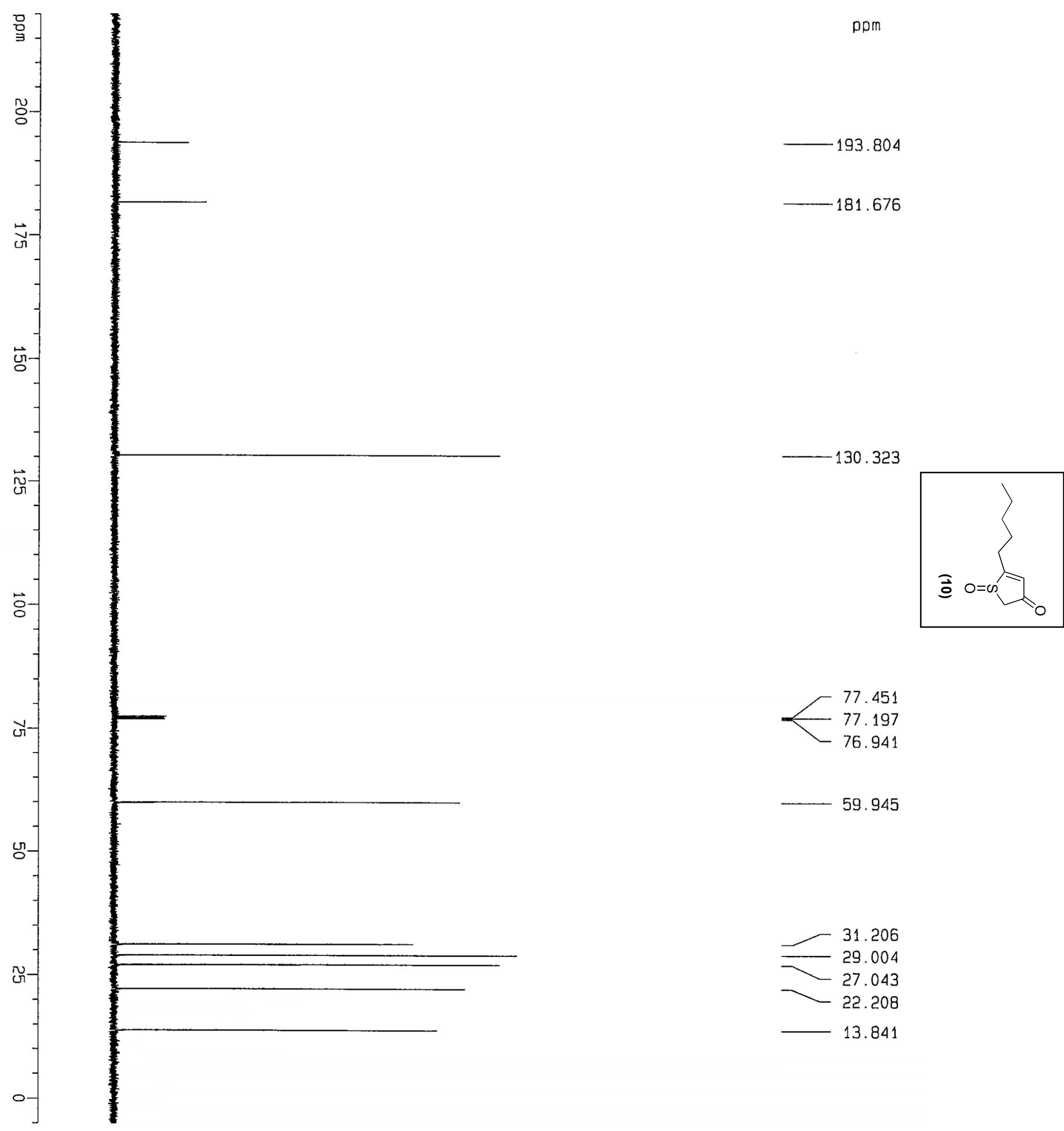

31.206
$-\quad 29.004$
-27.043
-22.208
-
13.841 


\section{${ }^{1} \mathrm{H}$ NMR Spectrum of 11}

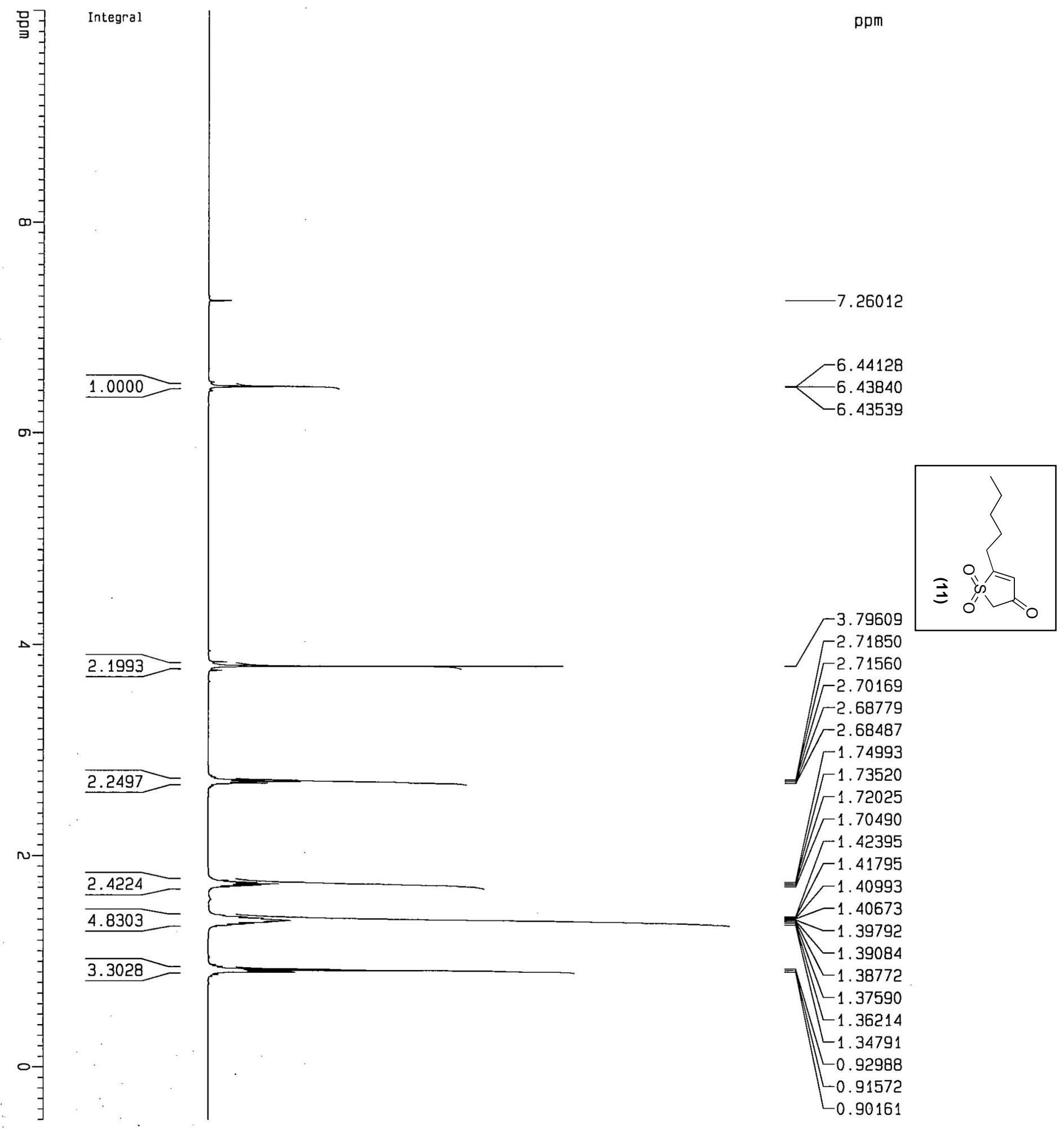




\section{${ }^{13} \mathrm{C}$ NMR Spectrum of 11}
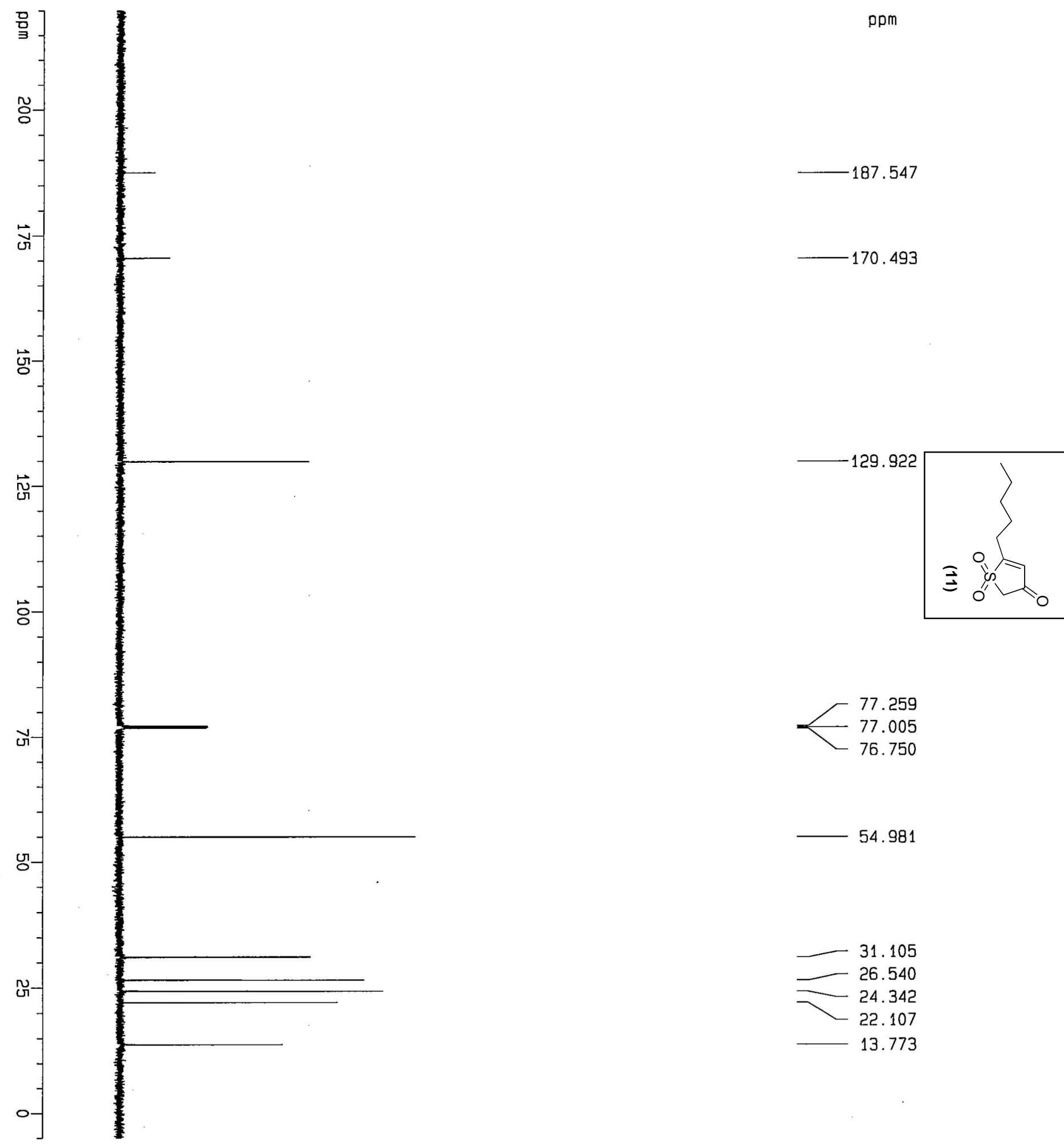


\section{${ }^{1}$ H NMR Spectrum of trans-Sulfoxide 12}

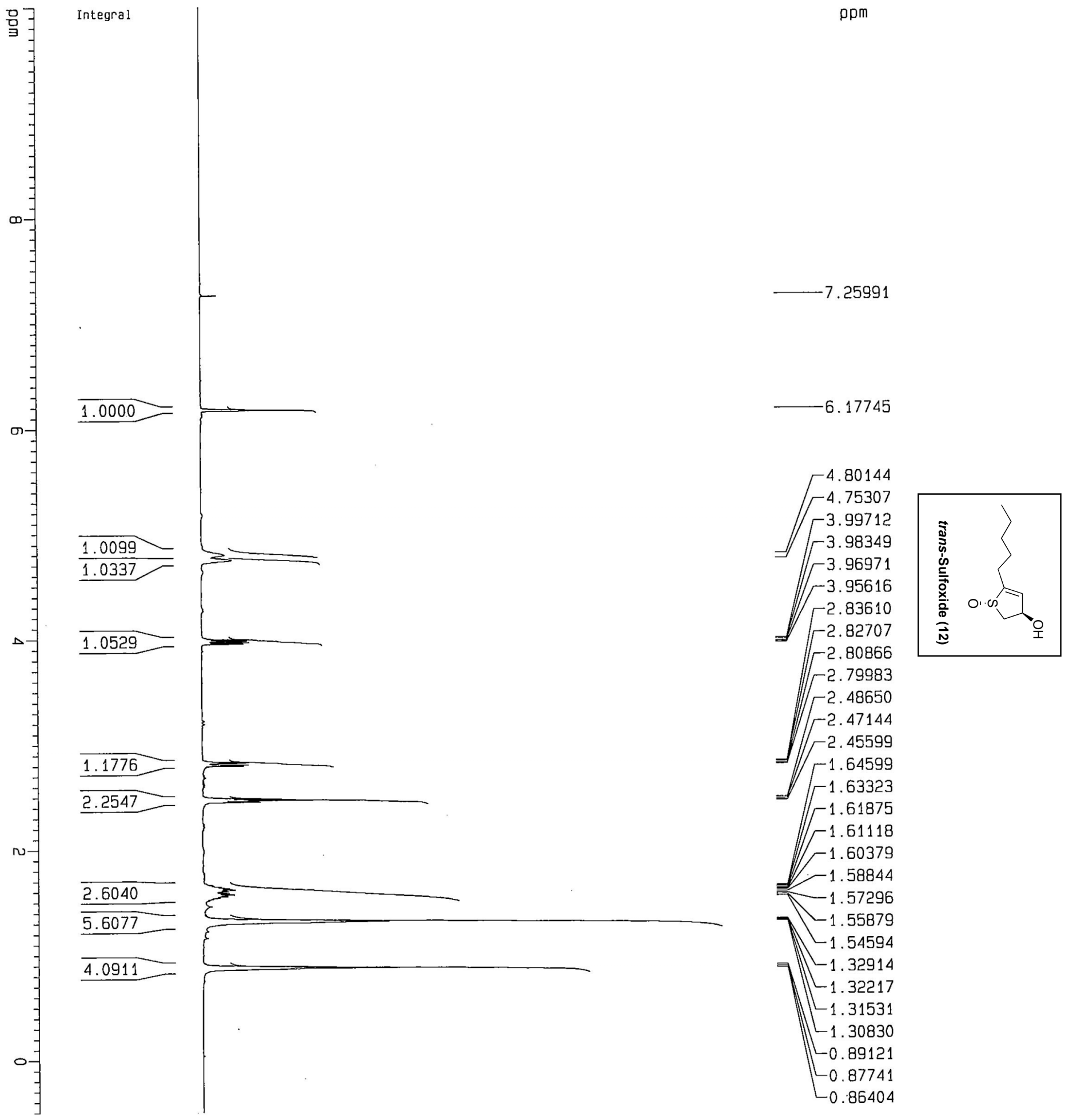




\section{${ }^{13}$ C NMR Spectrum of trans-Sulfoxide 12}

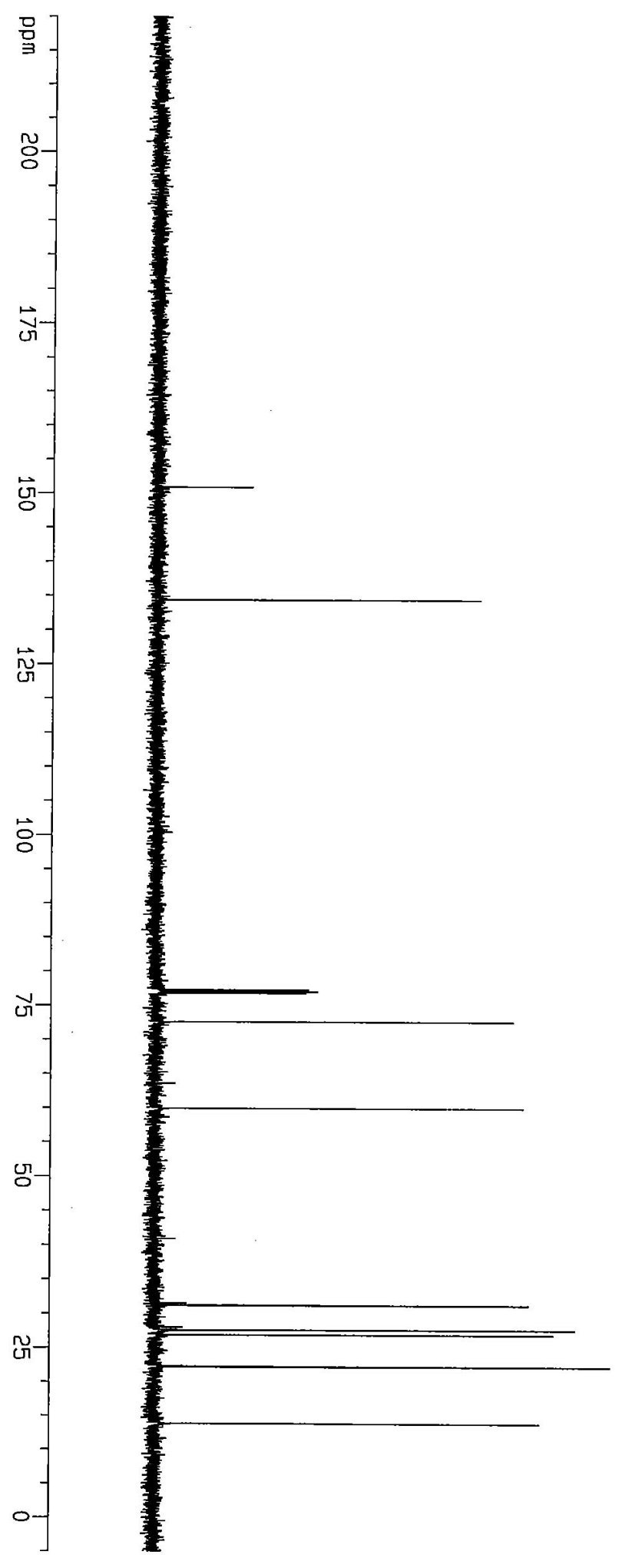

ppm

150.888

134.340

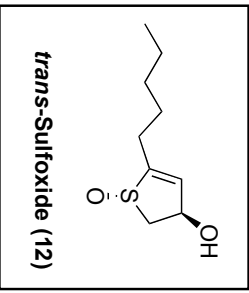

77.253

76.999

76.744

72.564

59.880

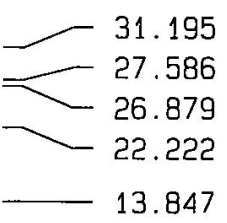




\section{${ }^{1}$ H NMR Spectrum of cis-Sulfoxide 12}

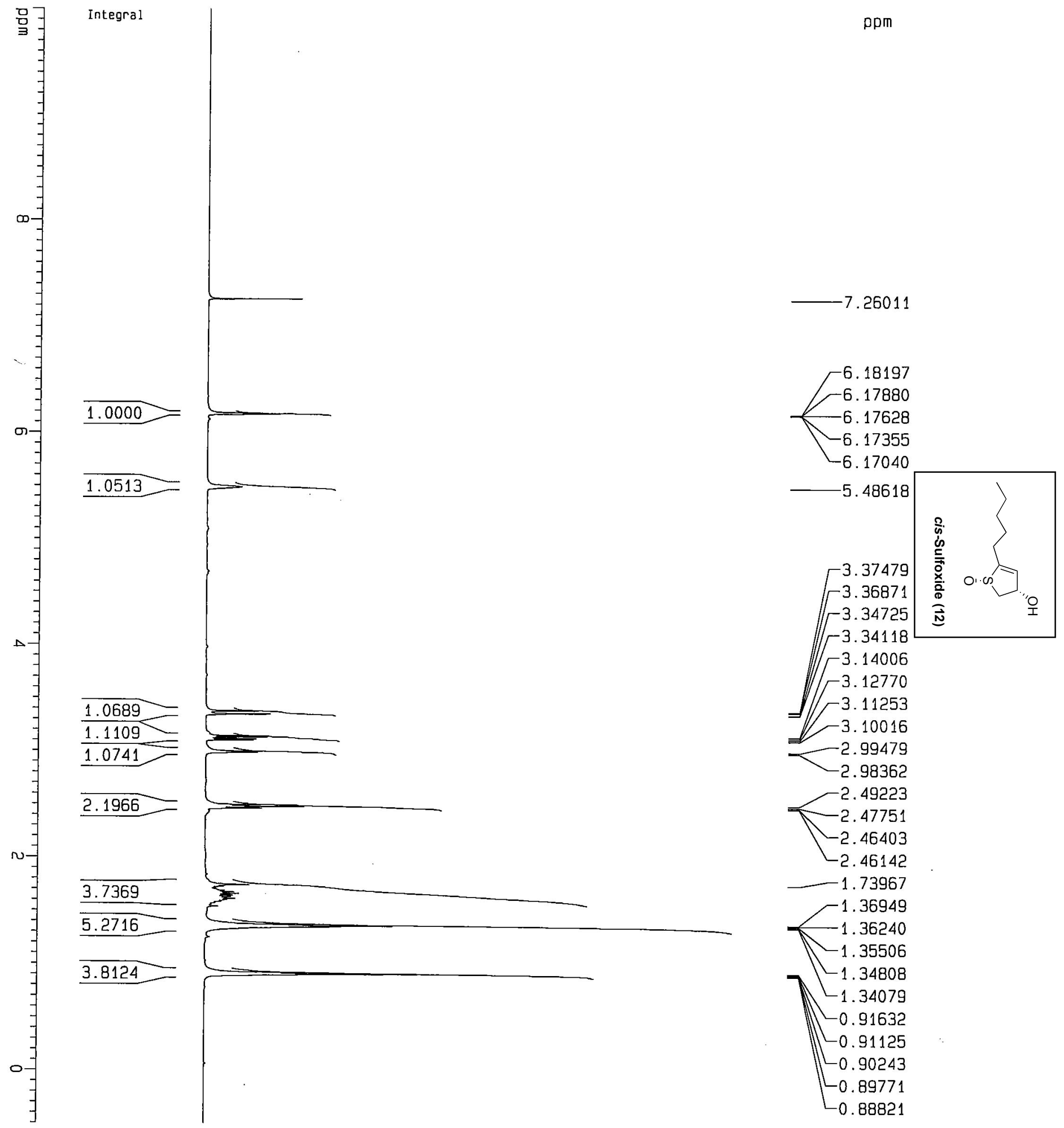




\section{${ }^{13}$ C NMR Spectrum of cis-Sulfoxide 12}
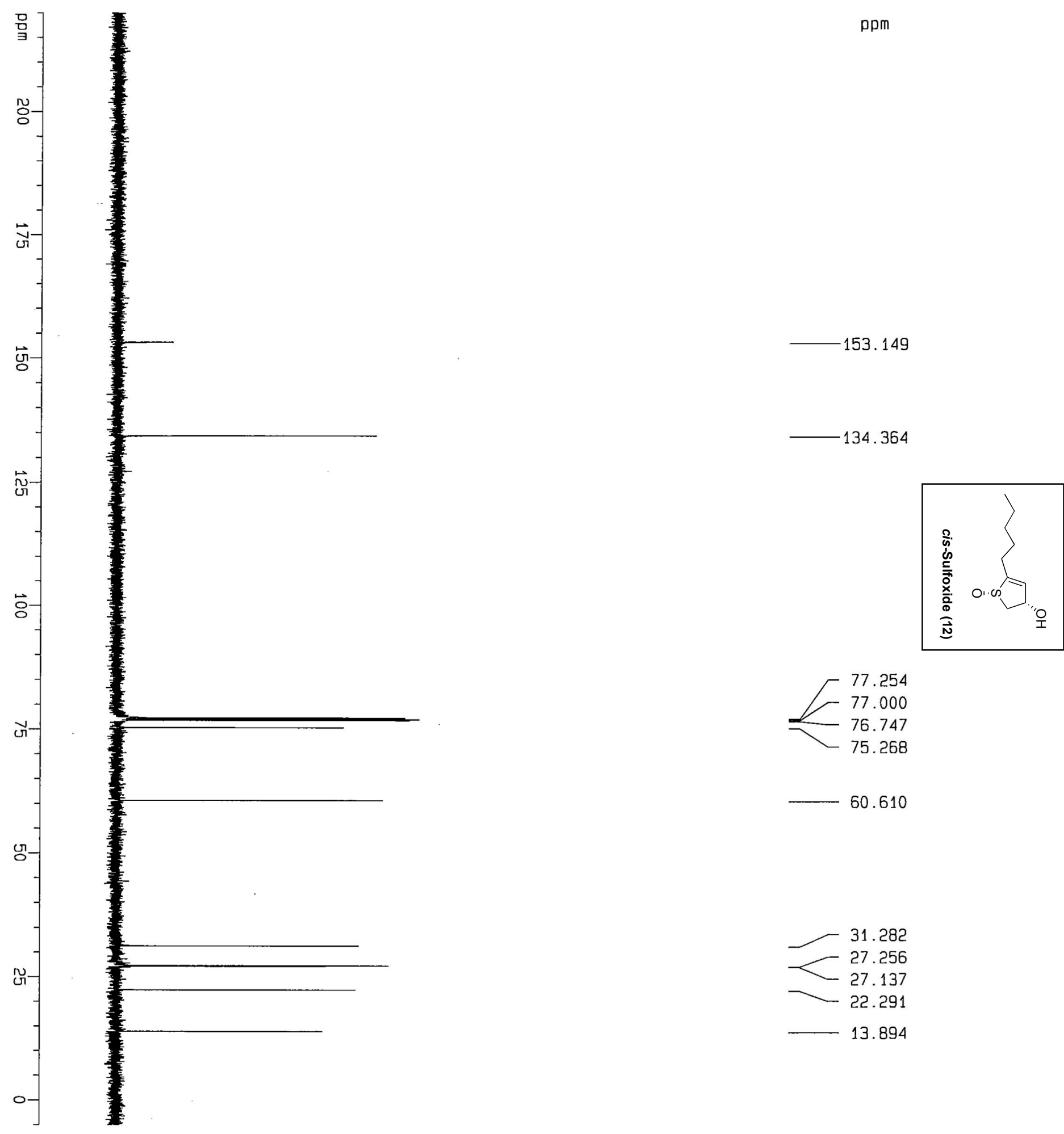


\section{${ }^{1} \mathrm{H}$ NMR Spectrum of 13}

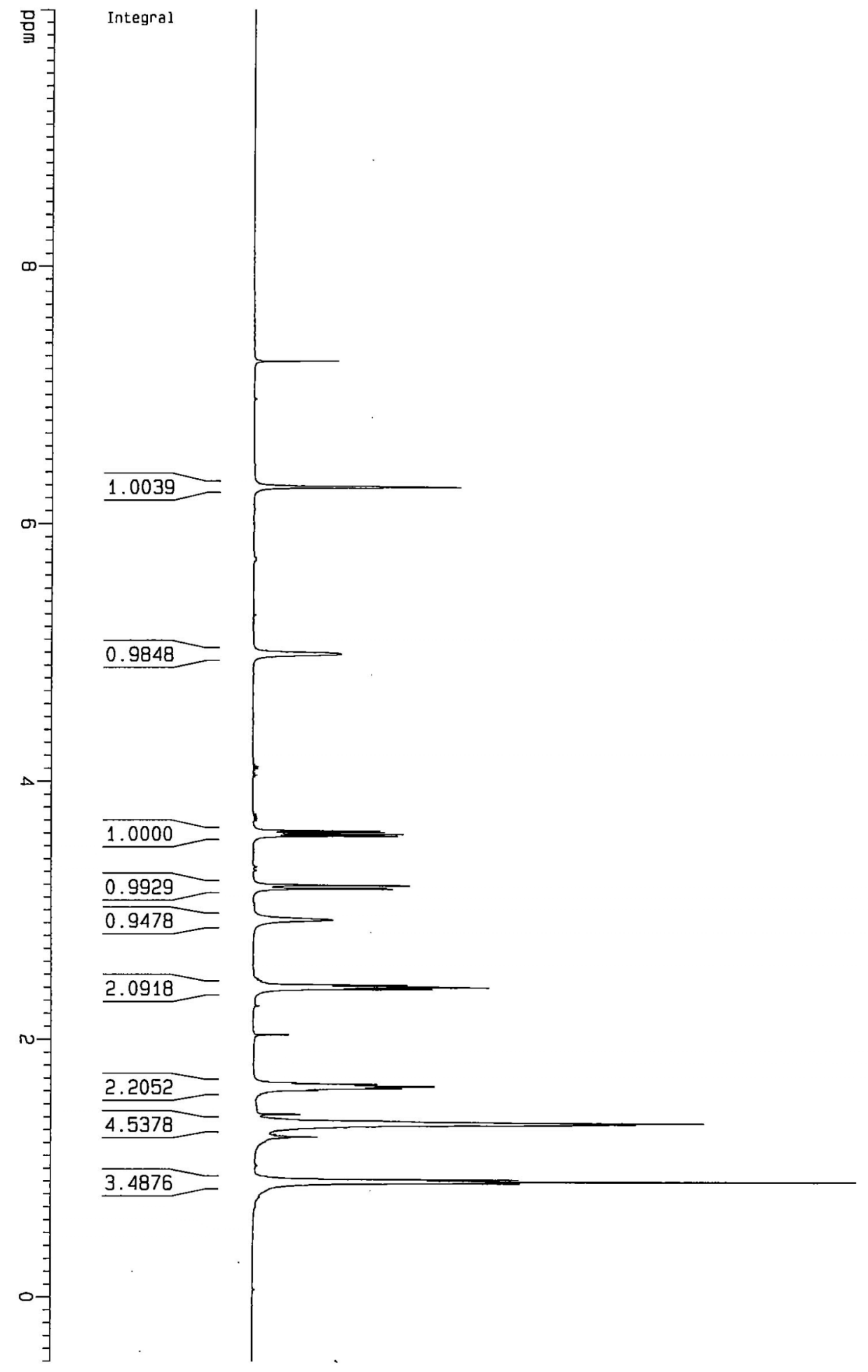

ppm
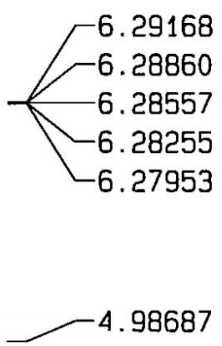

3.61896

$-3.60437$

$-3.59137$

$-3.57679$

3.19840

$-3.19169$

3.17082

$-3.16411$

2.92511

2.41846

$-2.41555$

$-2.40308$

$-2.39041$

2.38735

$-1.65110$

$-1.63596$

$-1.62084$

$-1.36708$

$-1.35970$

$7-1.35243$

$-1.34527$

$L_{1.33818}$

$L_{0.91266}$

$-0.90952$

$-0.89871$

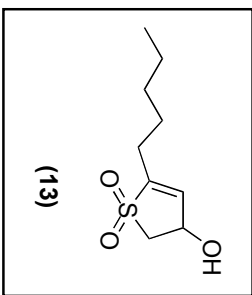

$-0.88443$ 


\section{${ }^{13}$ C NMR Spectrum of 13}

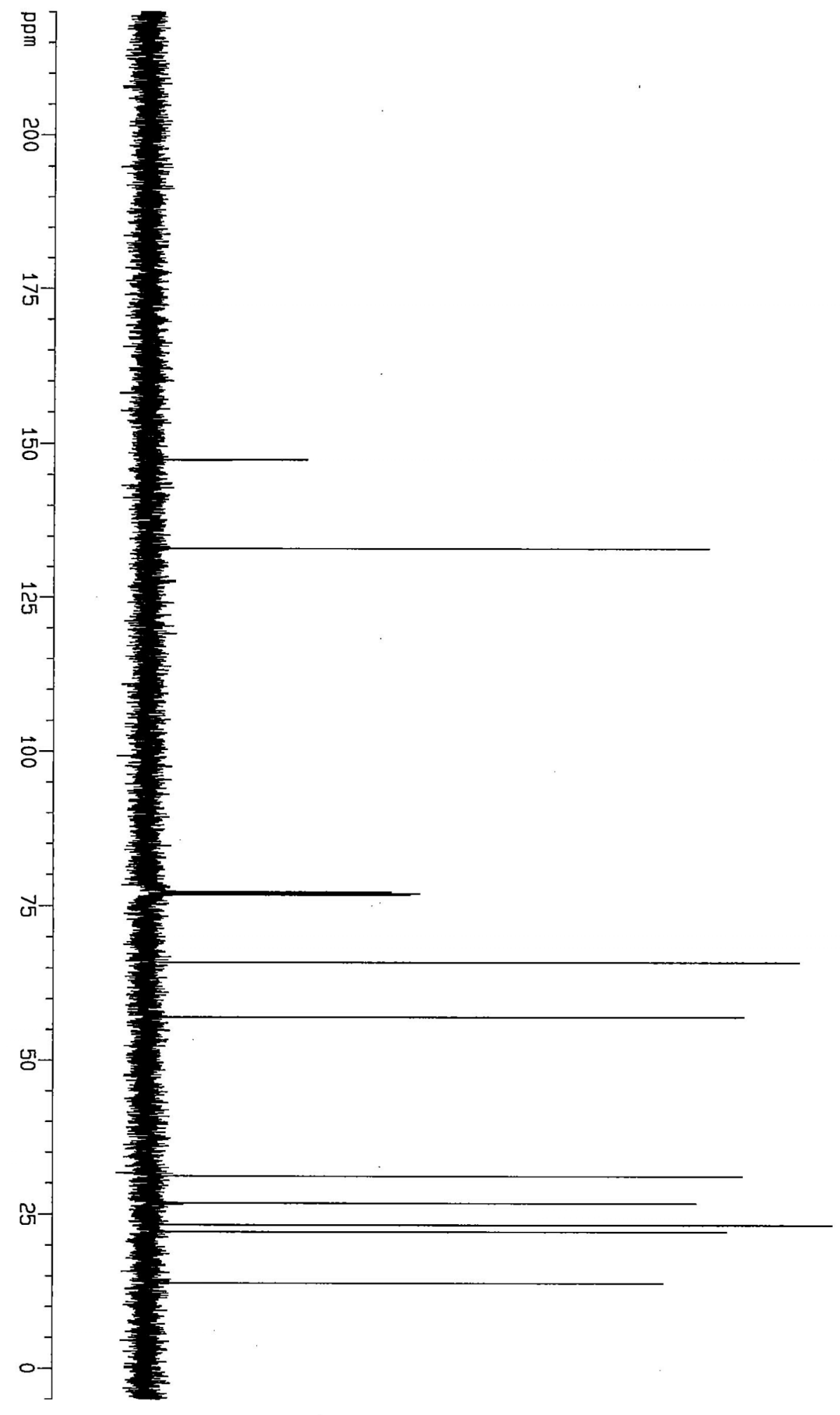

147.383

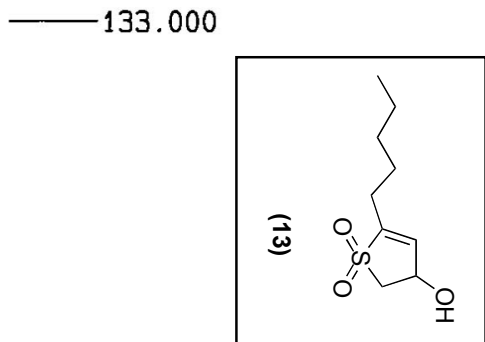

77.254

77.000

76.746

65.956

57.068

31.177

26.801

23. 289

22.175

13. 840 


\section{${ }^{1} \mathrm{H}$ NMR Spectrum of 14}

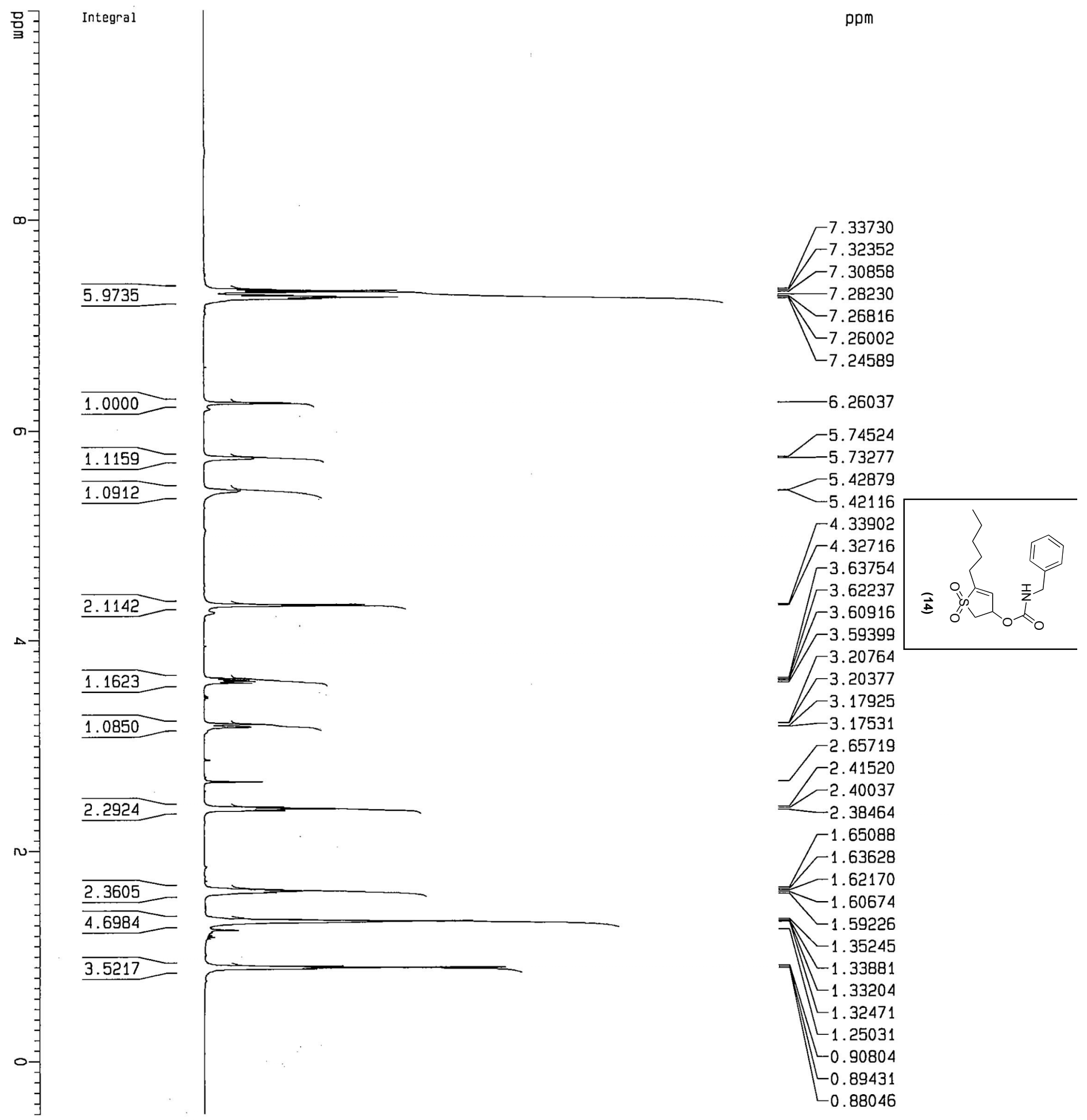




\section{${ }^{13}$ C NMR Spectrum of 14}
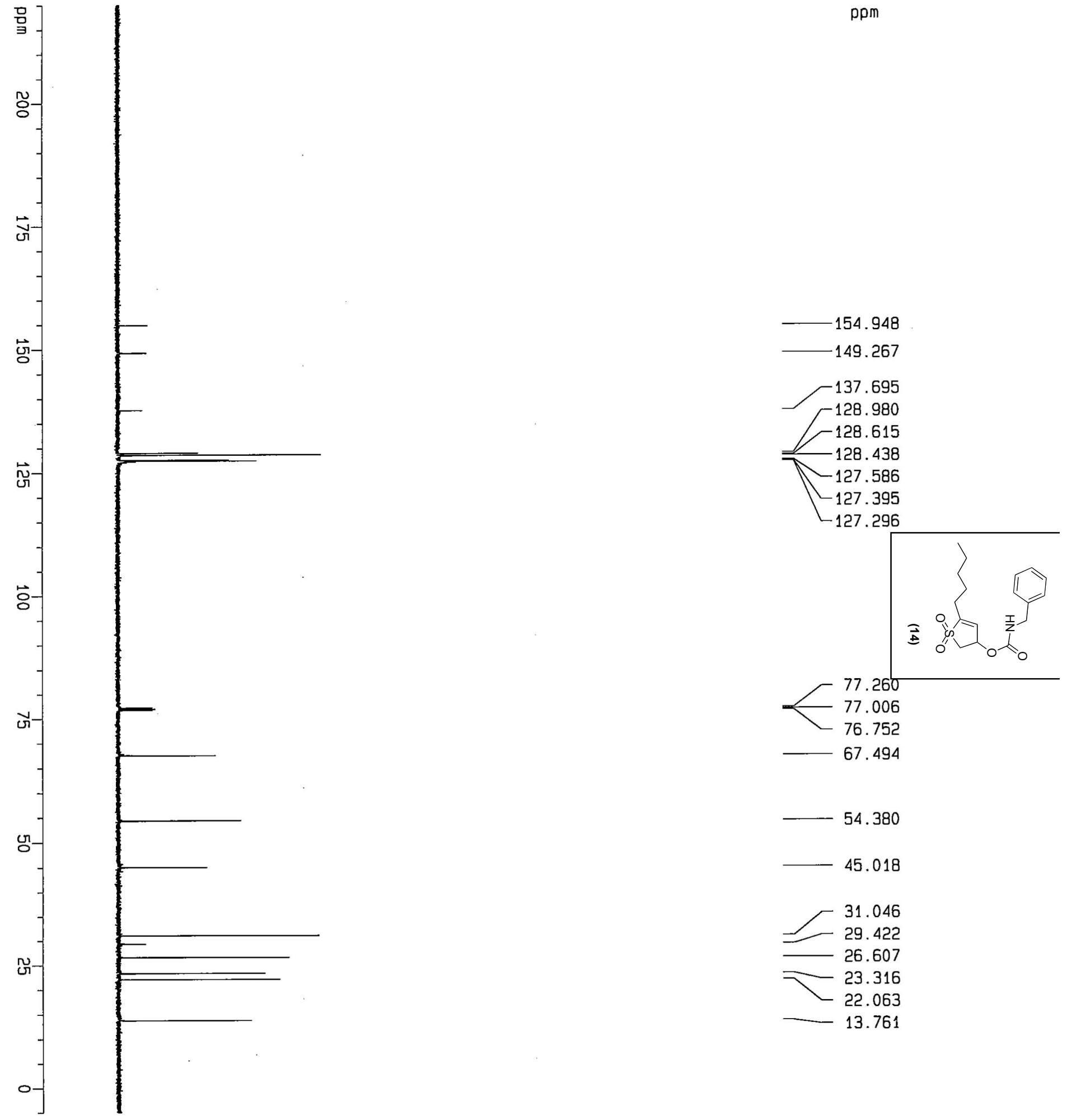


\section{${ }^{1}$ H NMR Spectrum of cis-Sulfoxide 14}
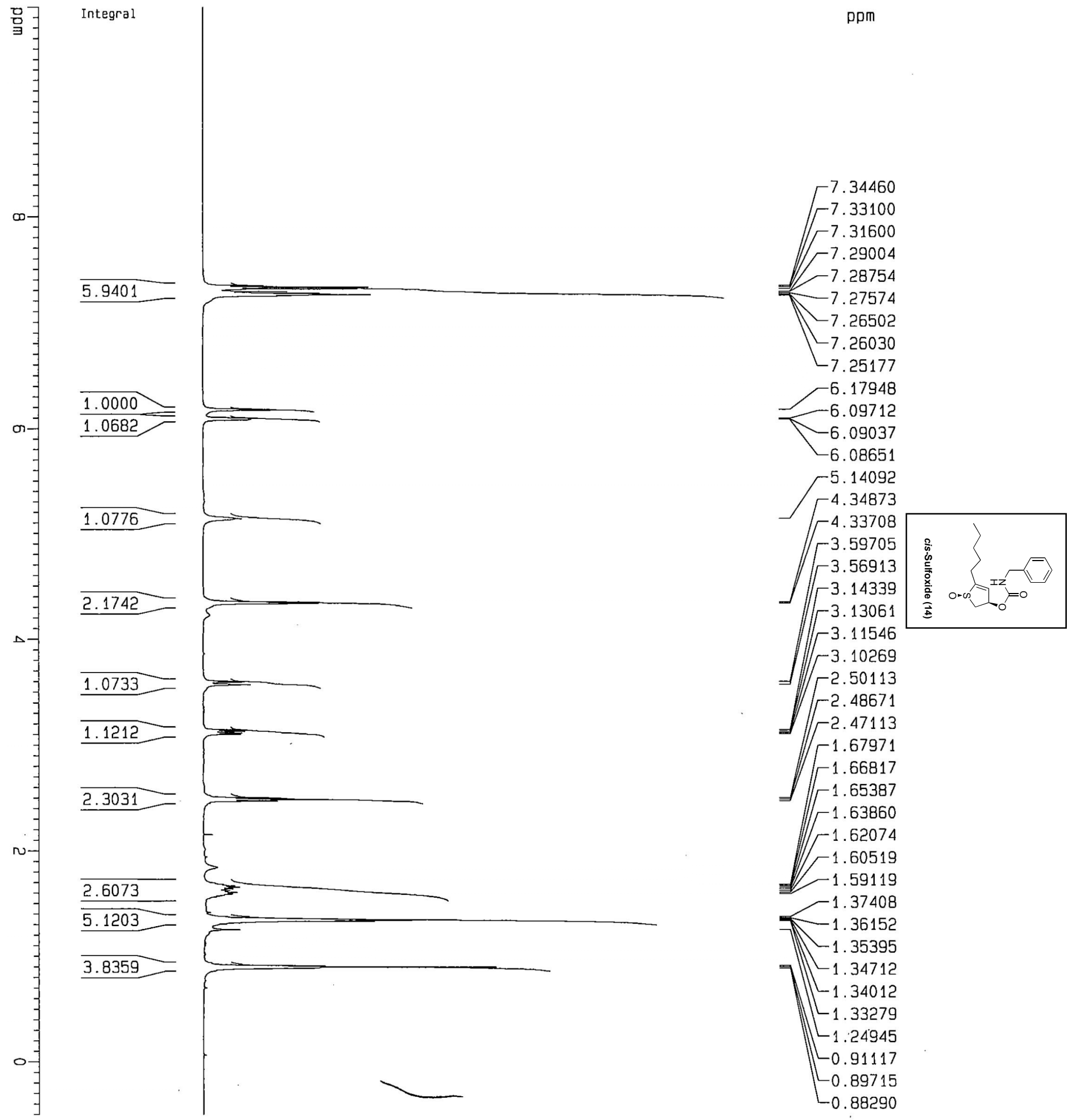

$-3.11546$

$-3.10269$

$-2.50113$

$-2.48671$

$-2.47113$

$-1.67971$

$-1.66817$

$=1.65387$

$-1.63860$

$-1.62074$

$-1.60519$

- -1.59119

$-1.37408$

$-1.36152$

$-1.35395$

7 -1.34712

$-1.34012$

$-1.33279$

$-1.24945$

$-0.91117$

$-0.89715$

$L_{0.88290}$ 


\section{${ }^{13}$ C NMR Spectrum of cis-Sulfoxide 14}
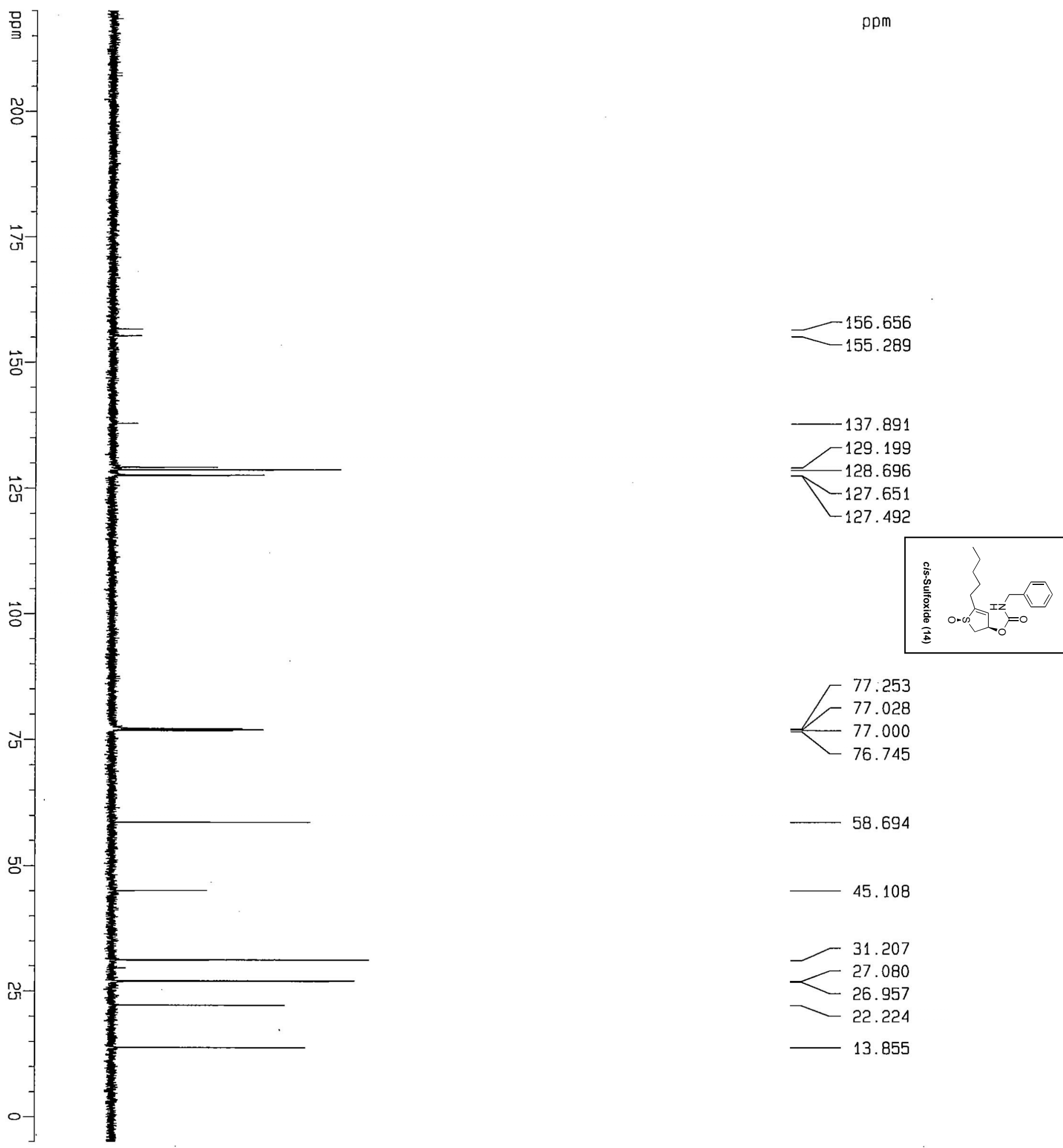


\section{${ }^{1}$ H NMR Spectrum of 2,3-cis-Sulfone 16}
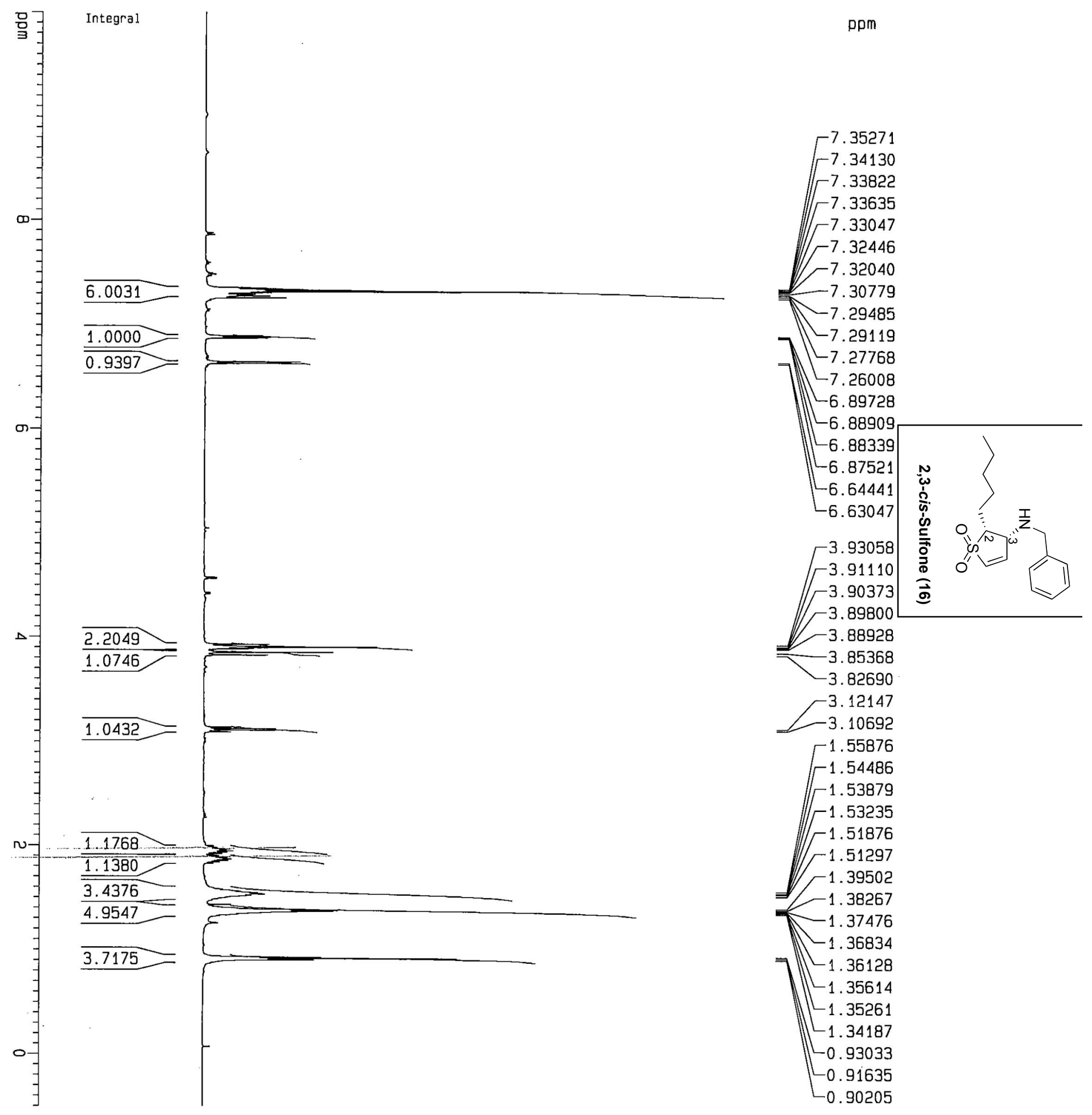


\section{${ }^{13}$ C NMR Spectrum of 2,3-cis-Sulfone 16}

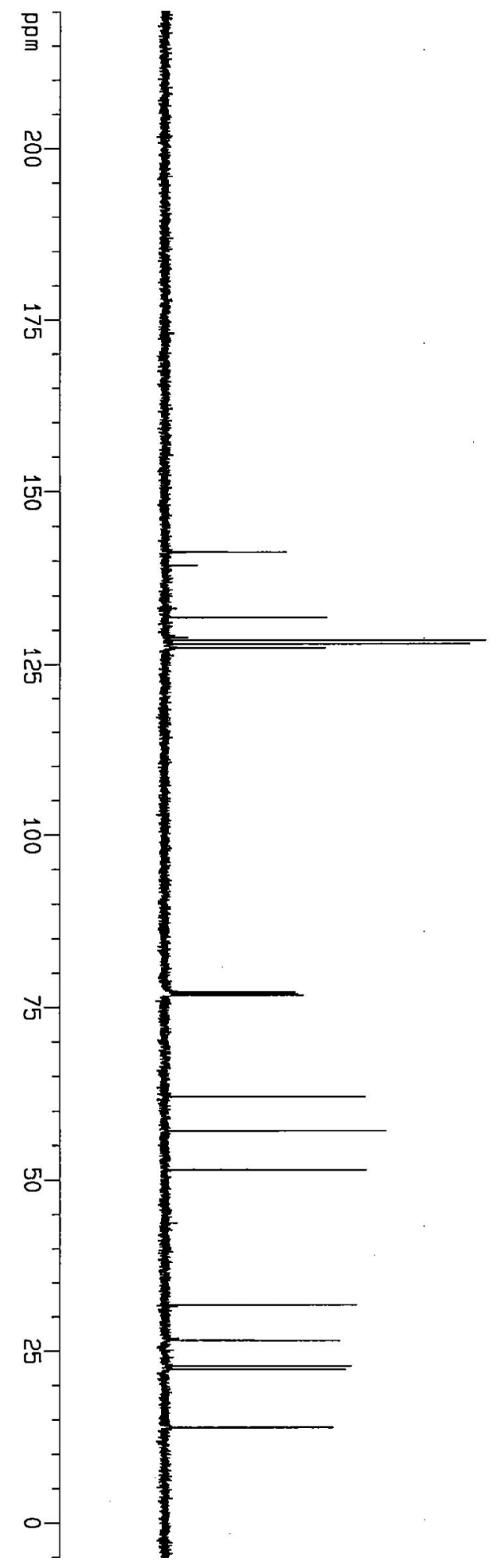

$\mathrm{ppm}$

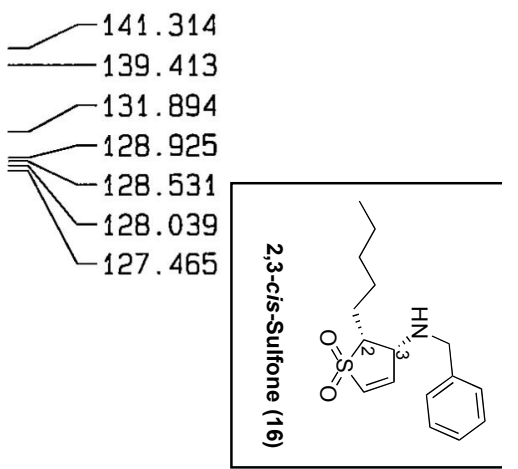

77.257

77.003

76.749

62.113

57.124

51.486

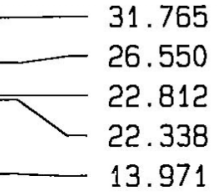




\section{${ }^{1}$ H NMR Spectrum of 2,3-trans-Sulfone 16}

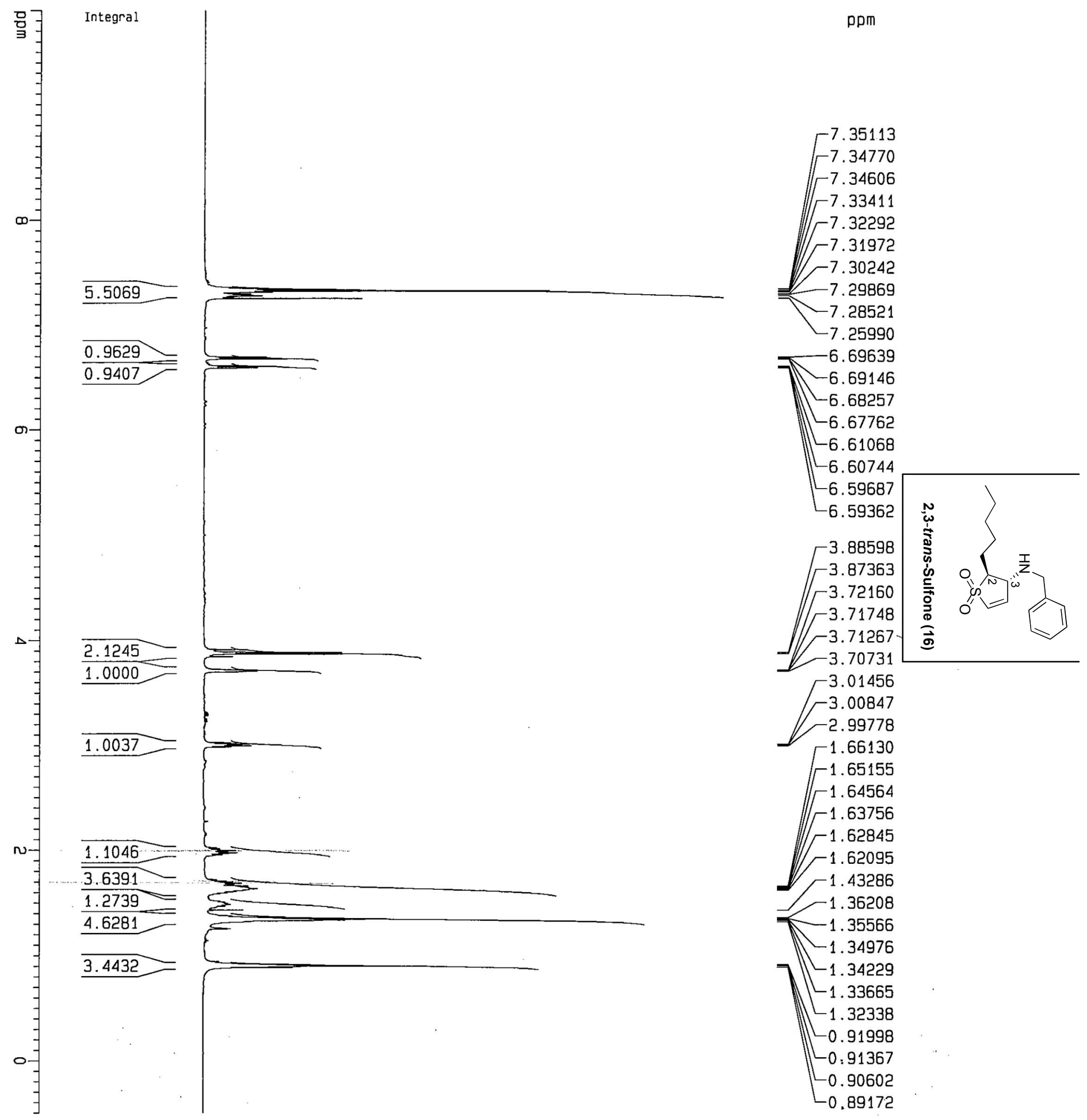




\section{${ }^{13}$ C NMR Spectrum of 2,3-trans-Sulfone 16}

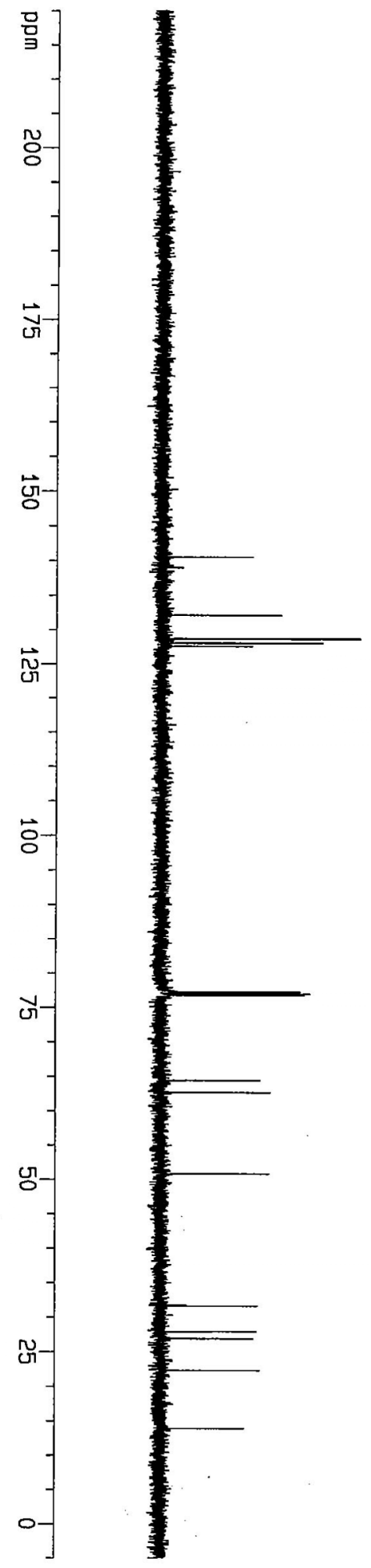

ppm
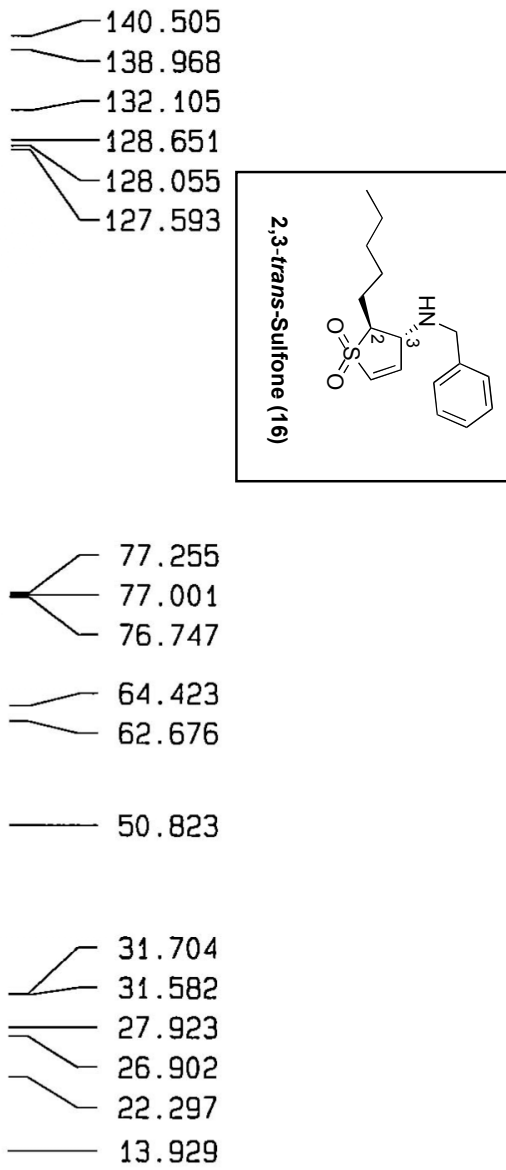


\section{${ }^{1}$ H NMR Spectrum of 2,3-cis-Sulfoxide 16}
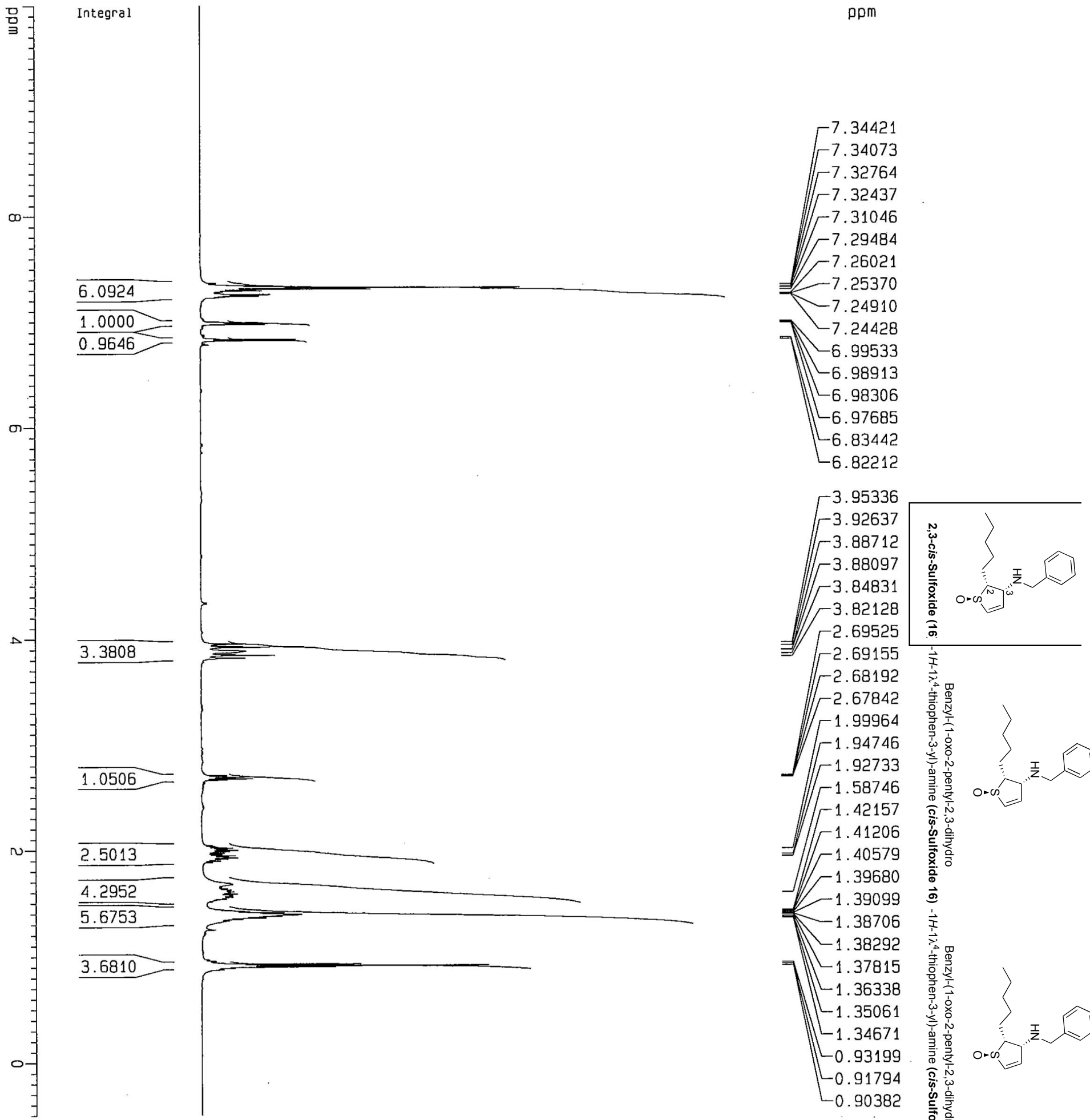


\section{${ }^{13}$ C NMR Spectrum of 2,3-cis-Sulfoxide 16}

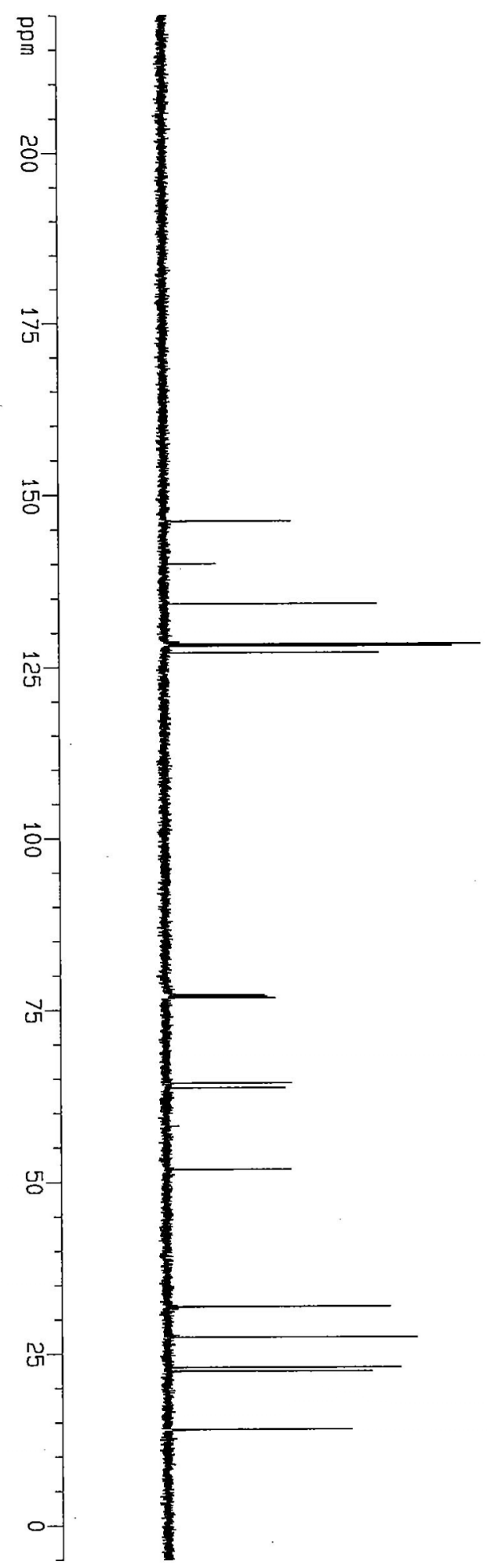

ppm
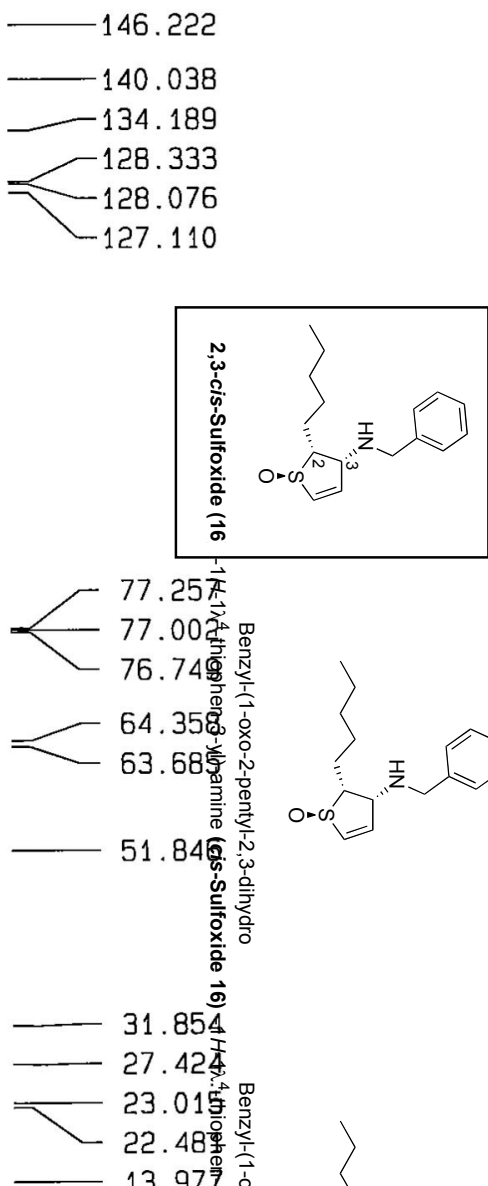


\section{${ }^{1}$ H NMR Spectrum of 2,3-trans-Sulfoxide 16}
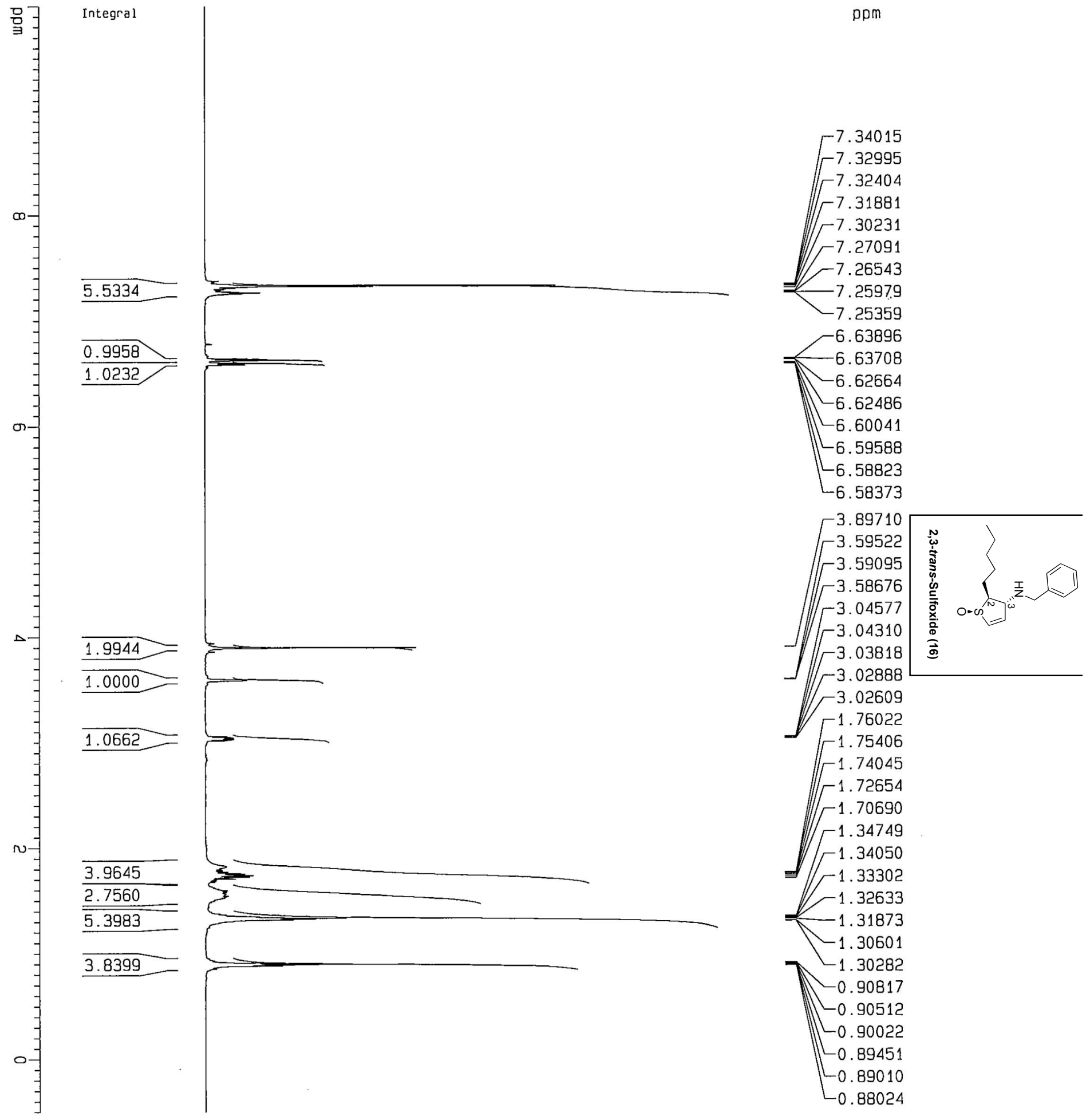

$-3.02609$

1.76022

$-1.75406$

$-1.74045$

$-1.72654$

$\Gamma^{1.70690}$

$-1.34749$

If ${ }^{-1.34050}$

3.9645

2.7560

5.3983

3.8399

$\left[\begin{array}{l}1.33302 \\ -1.32633 \\ 1.31873 \\ -1.30601 \\ 1.30282 \\ 0.90817 \\ 0.90512 \\ 0.90022 \\ 0.89451 \\ 0.89010 \\ 0.88024\end{array}\right.$ 


\section{${ }^{13}$ C NMR Spectrum of 2,3-trans-Sulfoxide 16}

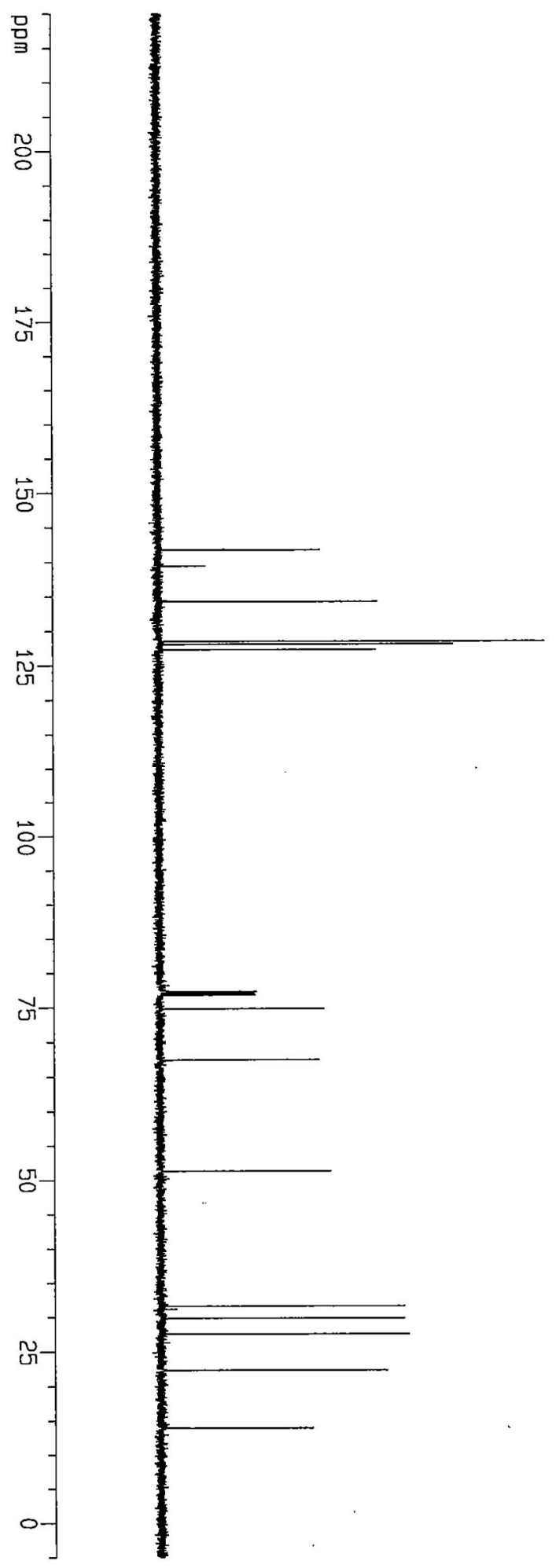

ppm
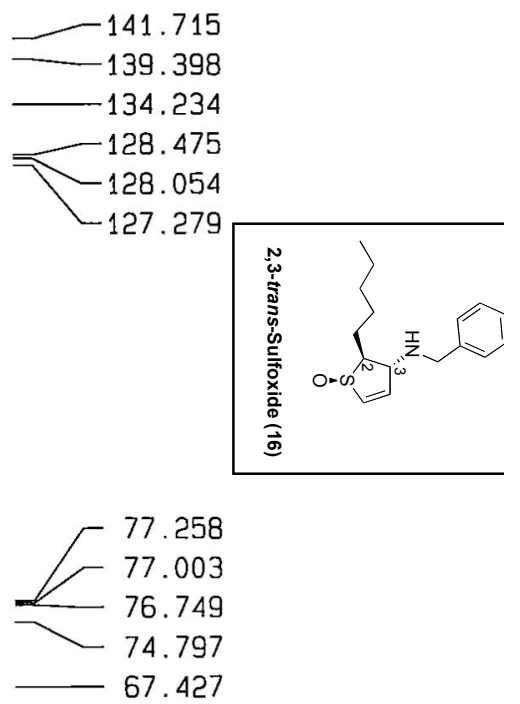

51.305

- 31.581

29.857

27.553

22. 290

13.888 


\section{${ }^{1}$ H NMR Spectrum of 2,3-trans-Sulfoxide 16a}

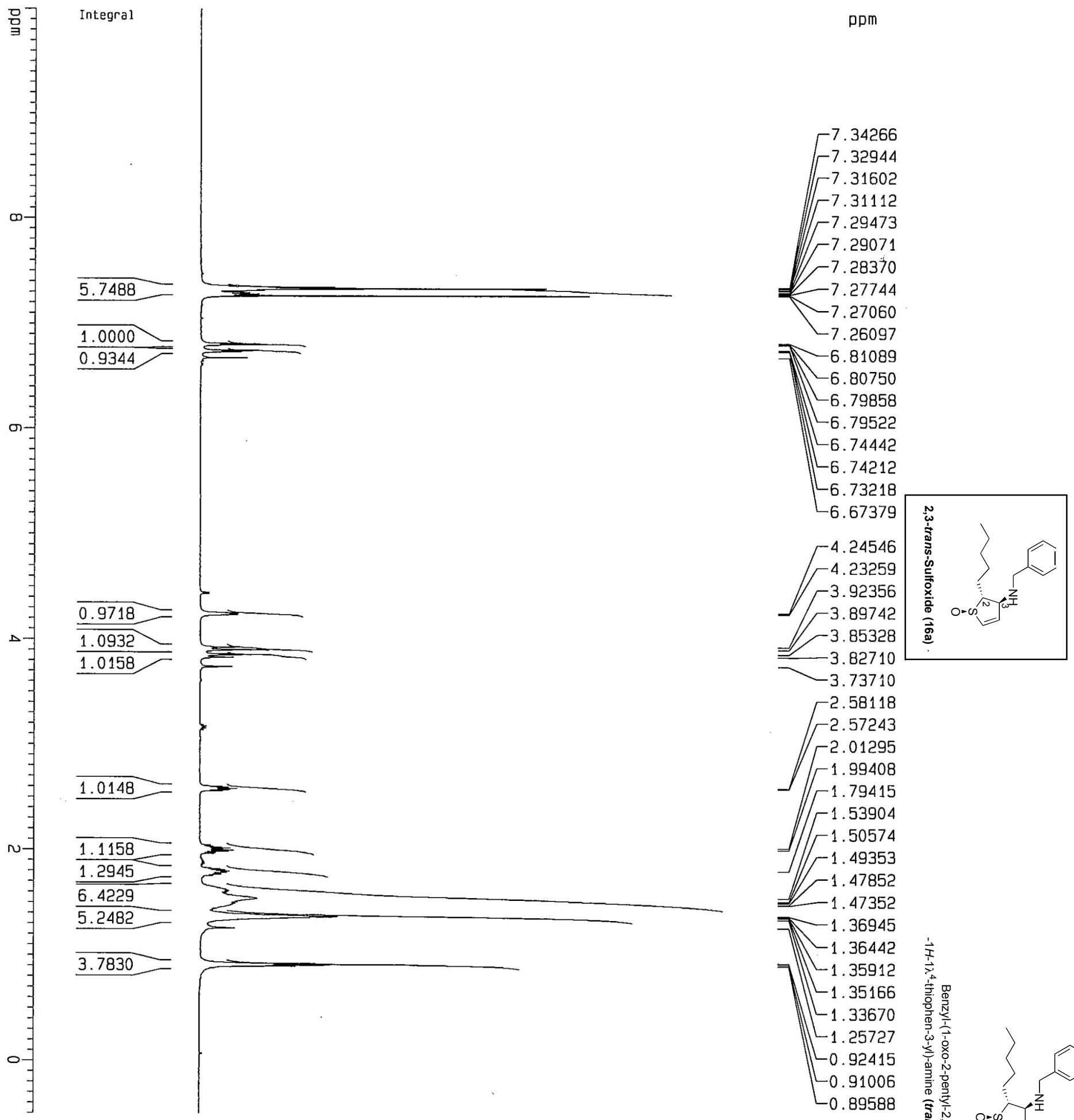




\section{${ }^{13} \mathrm{C}$ NMR Spectrum of 2,3-trans-Sulfoxide 16a}

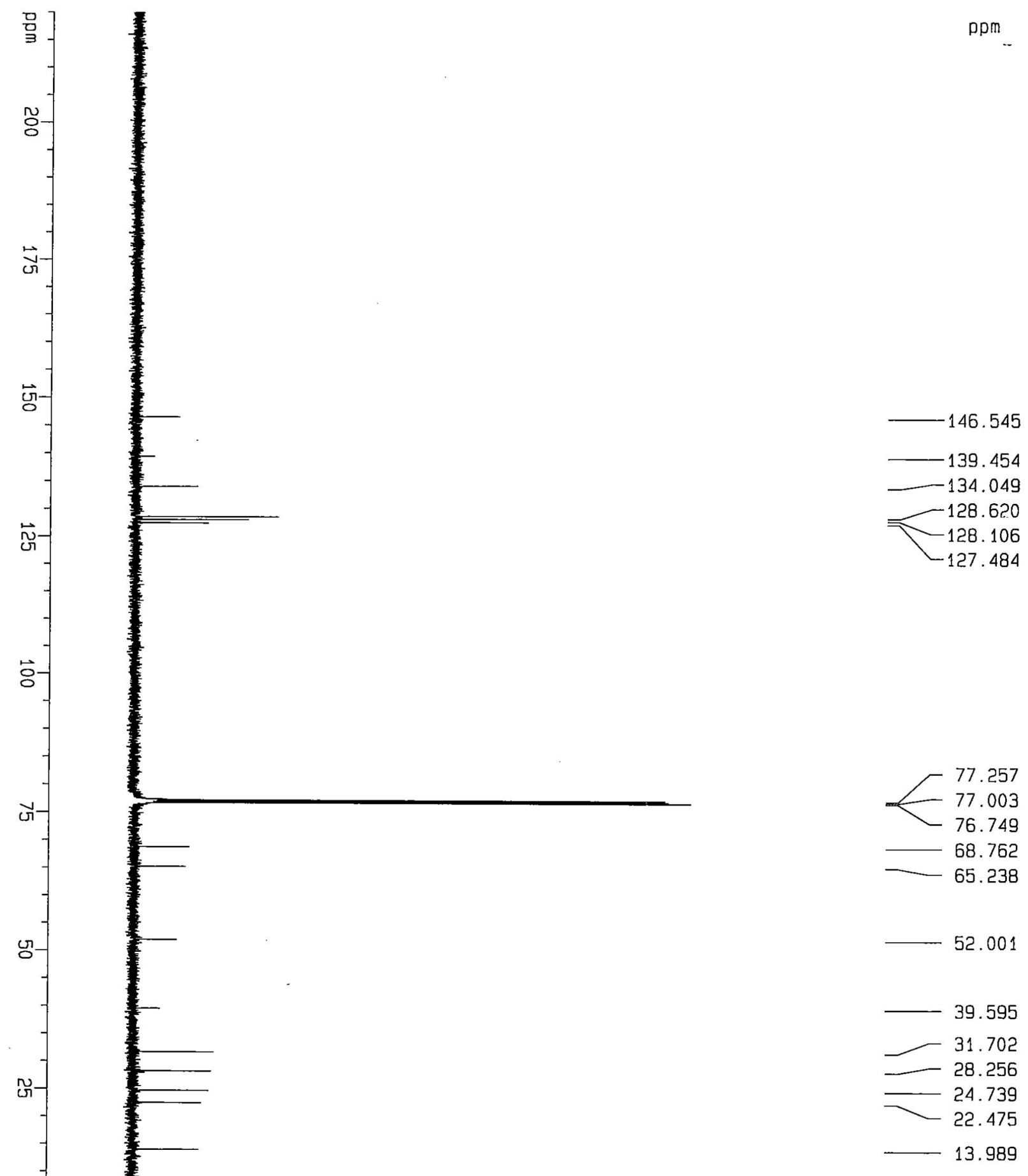




\section{${ }^{1}$ H NMR Spectrum of 2,3-cis-Sulfoxide 16a}
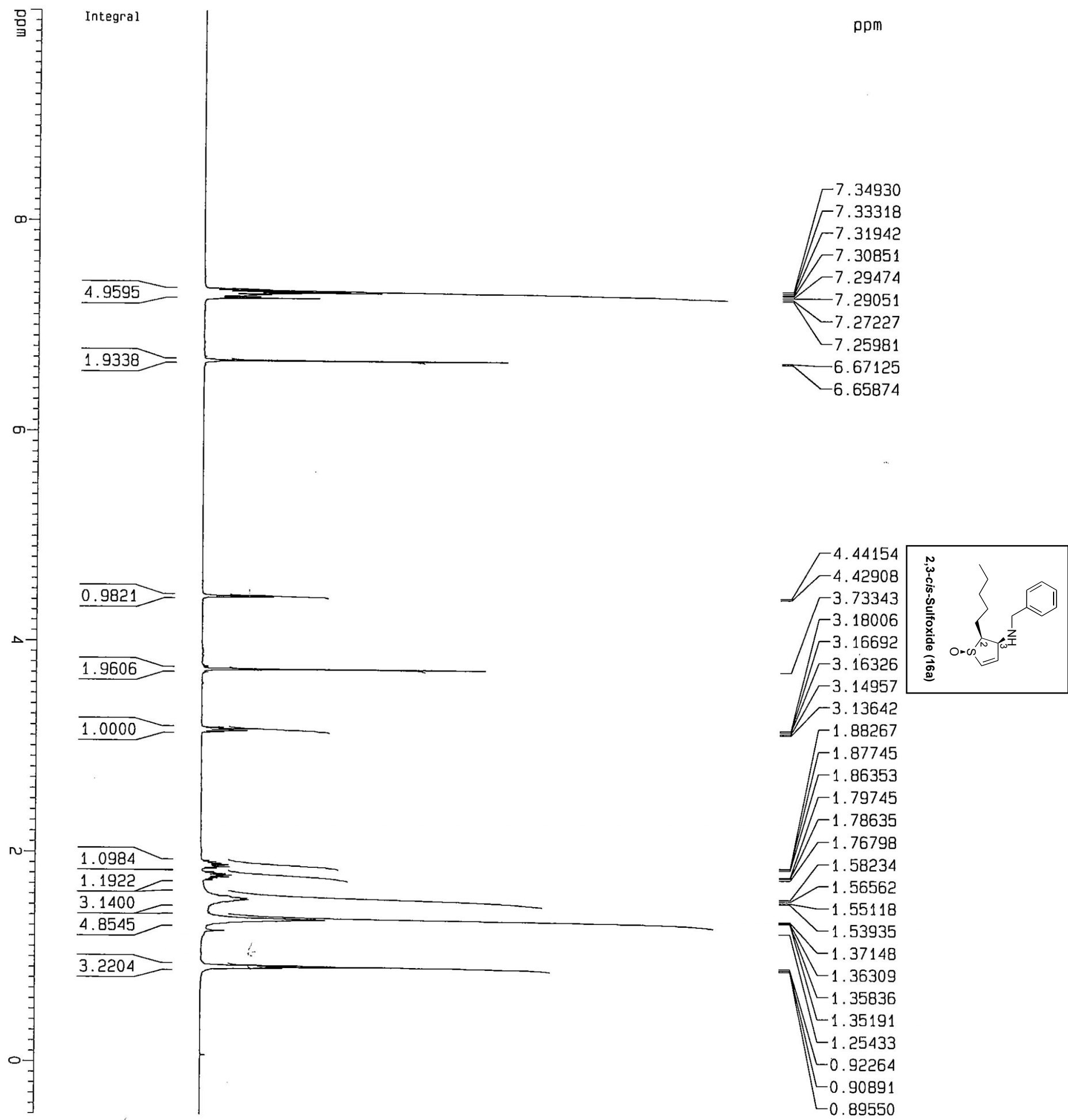

$-3.13642$

1.88267

$-1.87745$

$-1.86353$

1.79745

$-1.78635$

1.76798

$-1.58234$

$-1.56562$

$-1.55118$

$-1.53935$

$-1.37148$

$-1.36309$

$-1.35836$

$L_{1} .35191$

$-1.25433$

$-0.92264$

$-0.90891$

$-0.89550$ 


\section{${ }^{13}$ C NMR Spectrum of 2,3-cis-Sulfoxide 16a}
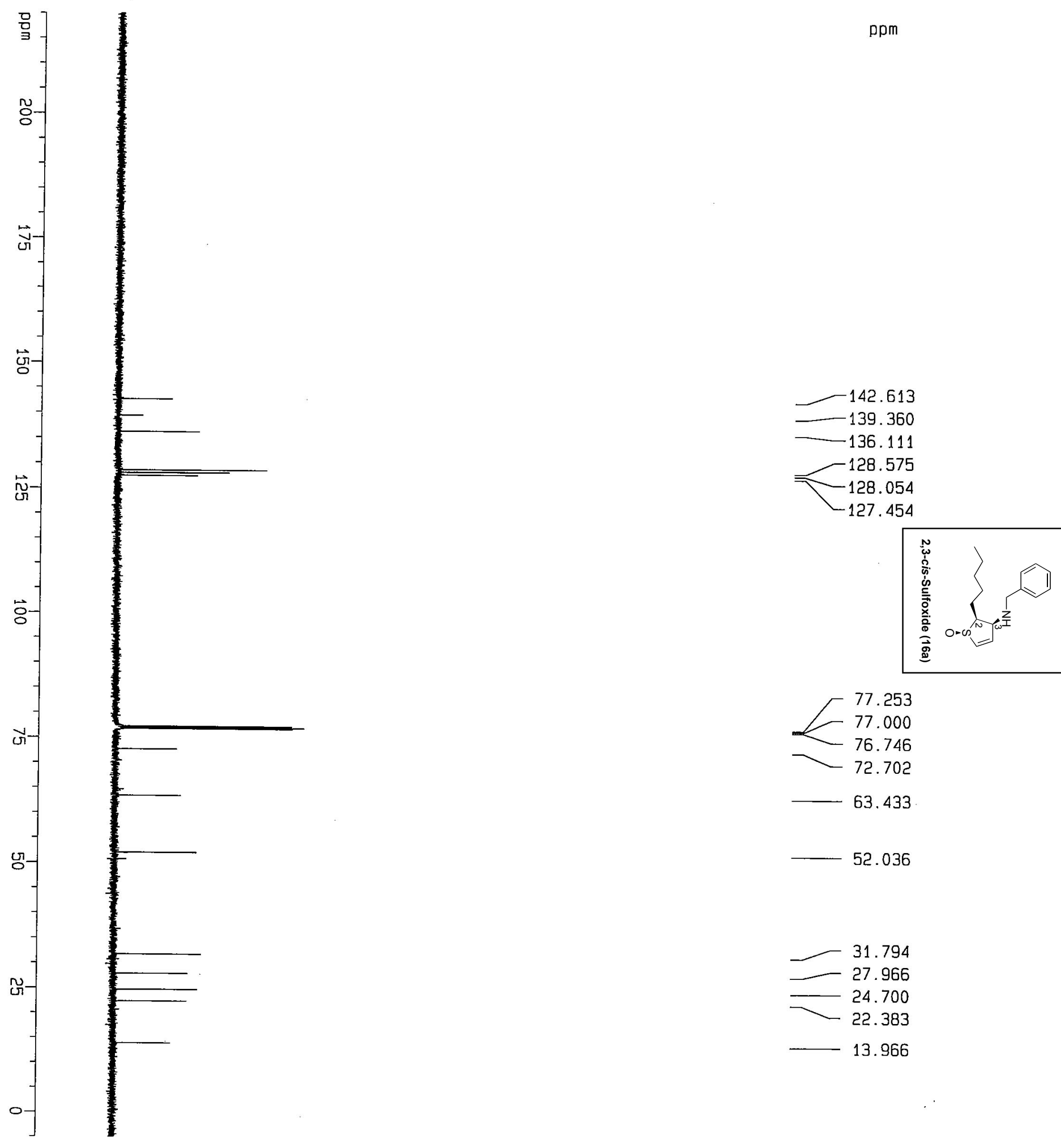


\section{${ }^{1}$ H NMR Spectrum of 2,3-cis-Sulfone 17}

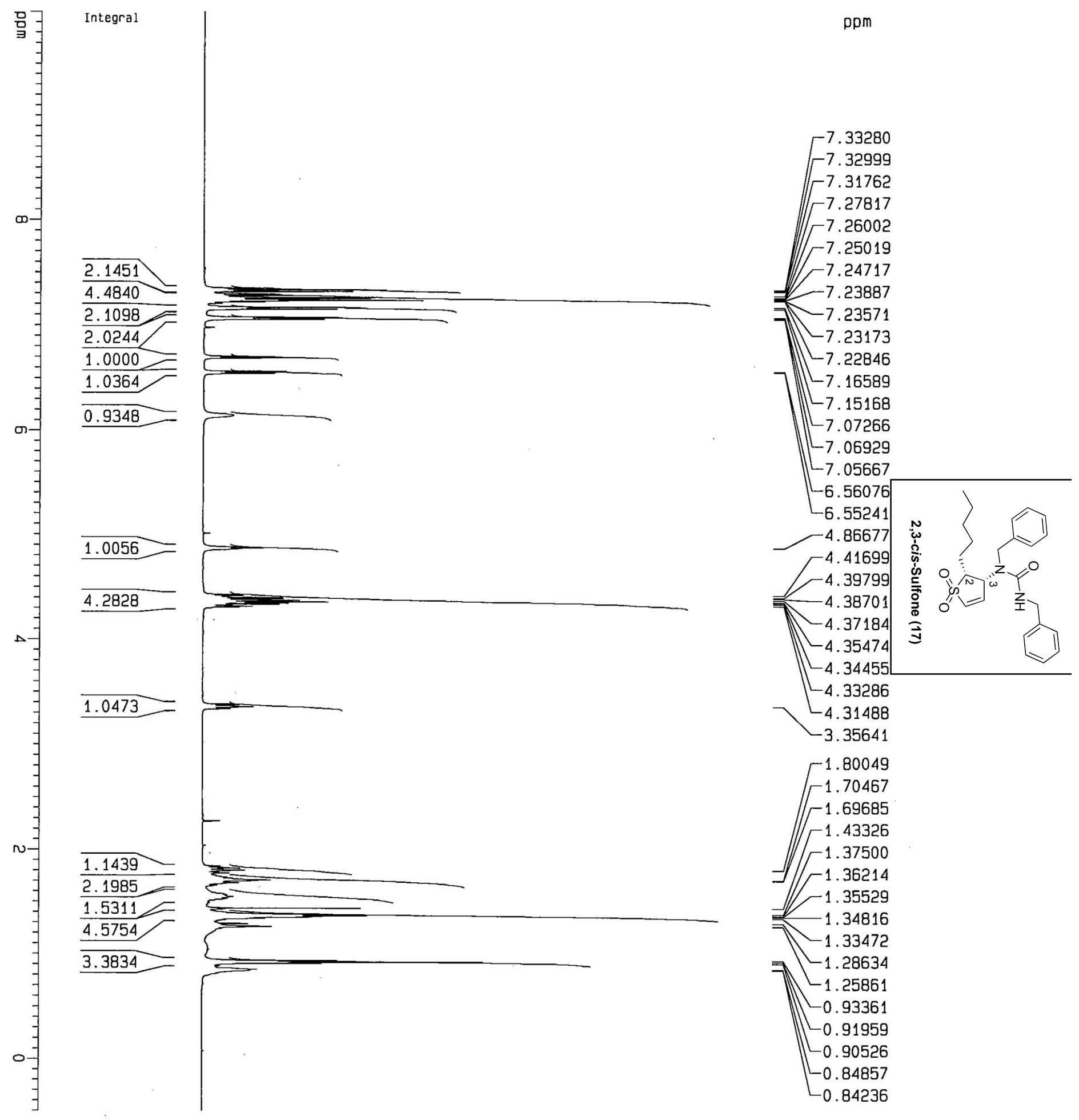




\section{${ }^{13}$ C NMR Spectrum of 2,3-cis-Sulfone 17}
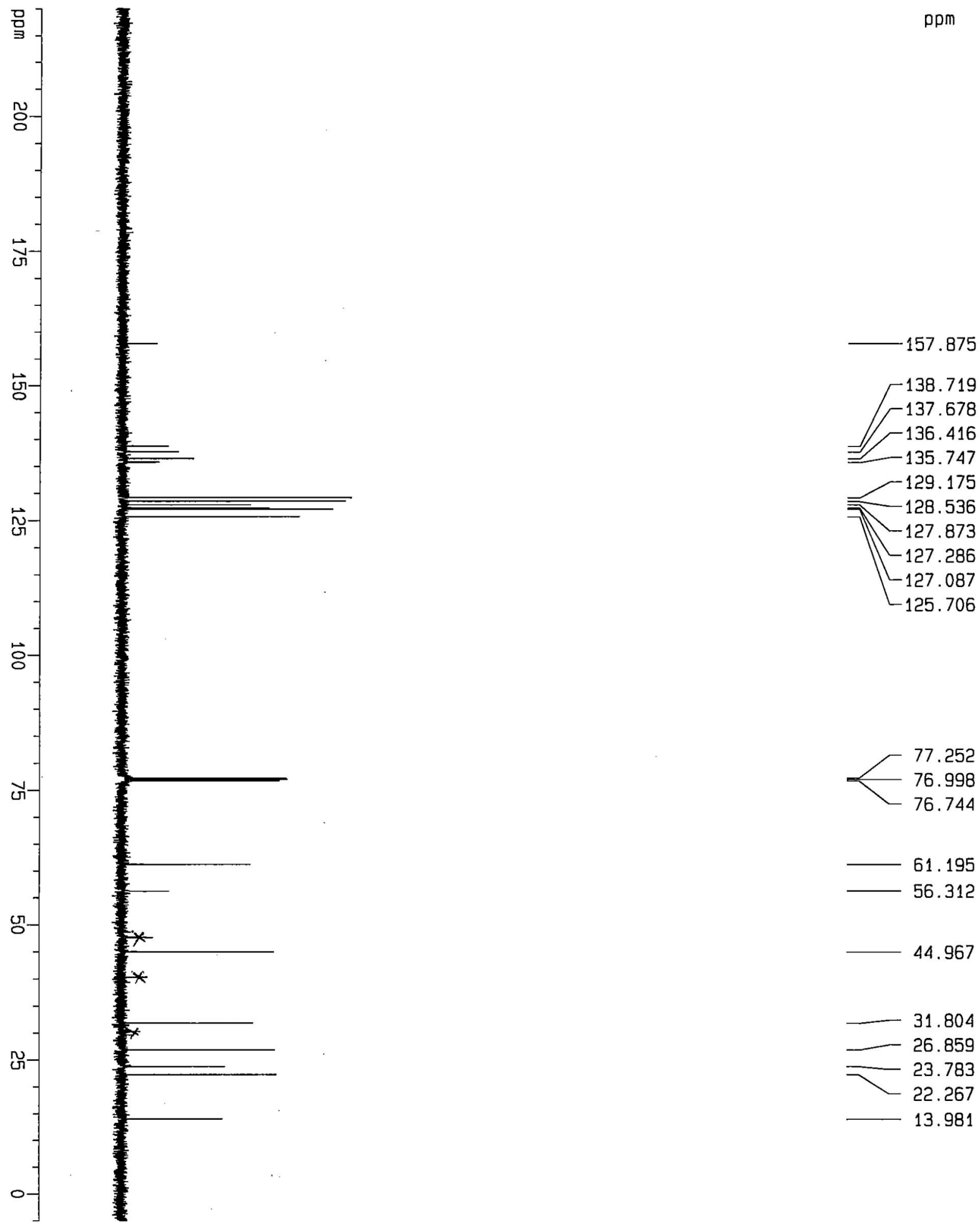


\section{${ }^{1}$ H NMR Spectrum of 2,3-trans-Sulfone 17}

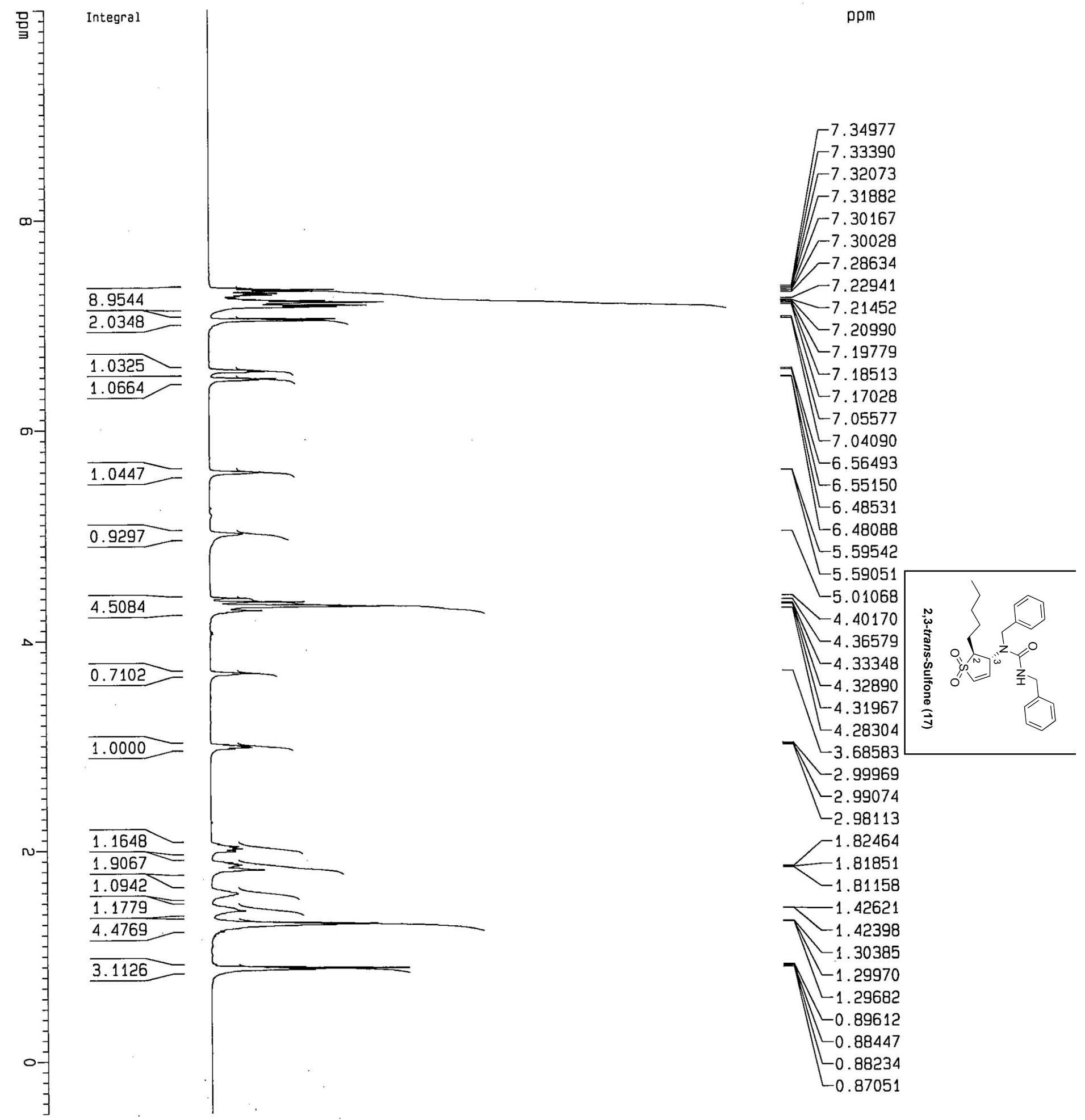




\section{${ }^{13}$ C NMR Spectrum of 2,3-trans-Sulfone 17}
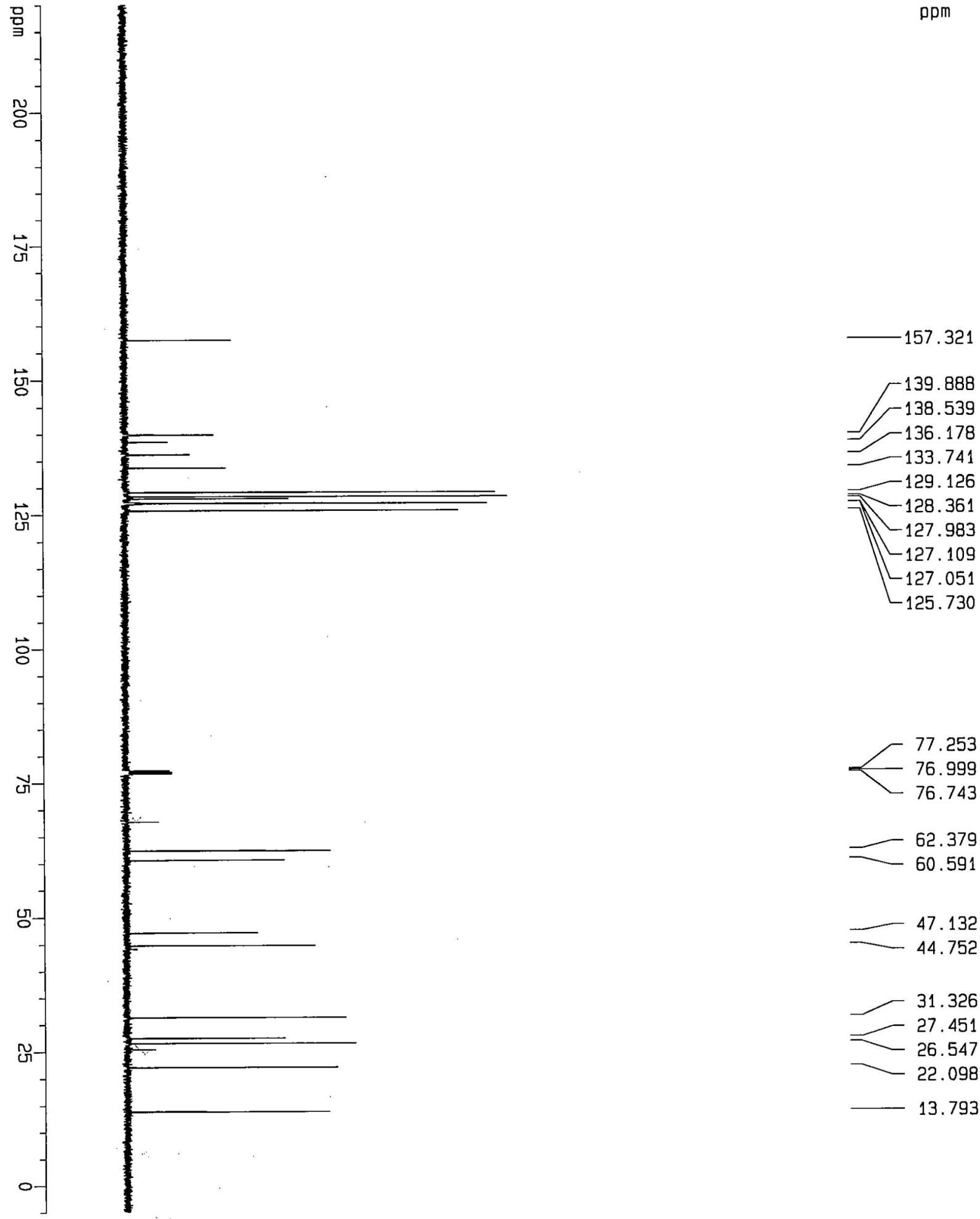

77.253

76.999

76.743

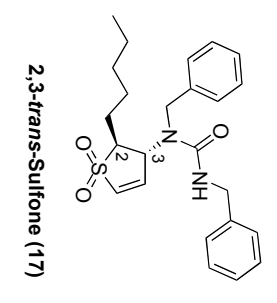

$-62.379$

60.591

-47.132
$-\quad 44.752$

$-31.326$

27.451

26.547

$-22.098$

13.793 


\section{${ }^{1}$ H NMR Spectrum of 2,3-trans-Sulfone 4}

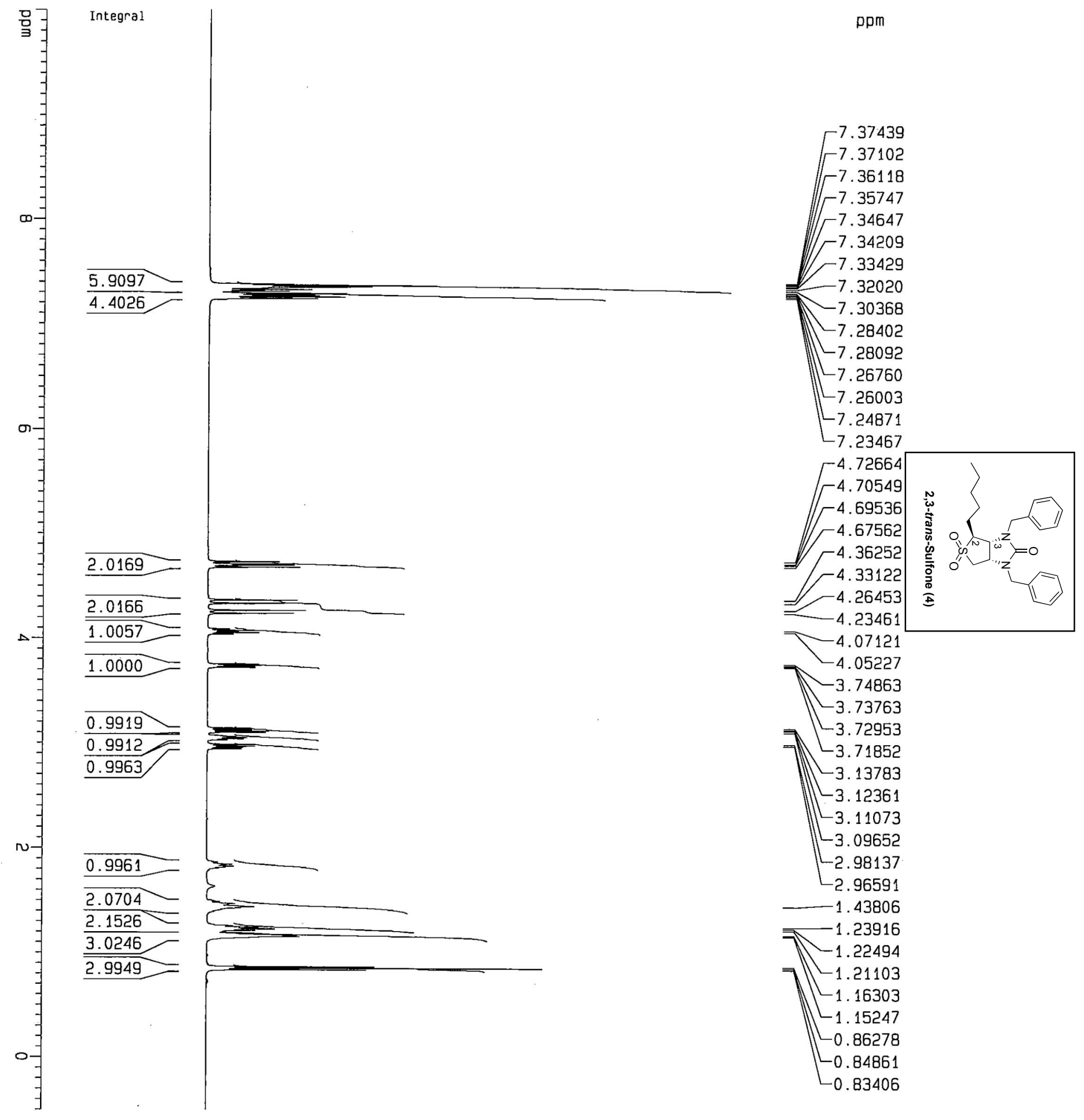




\section{${ }^{13}$ C NMR Spectrum of 2,3-trans-Sulfone 4}
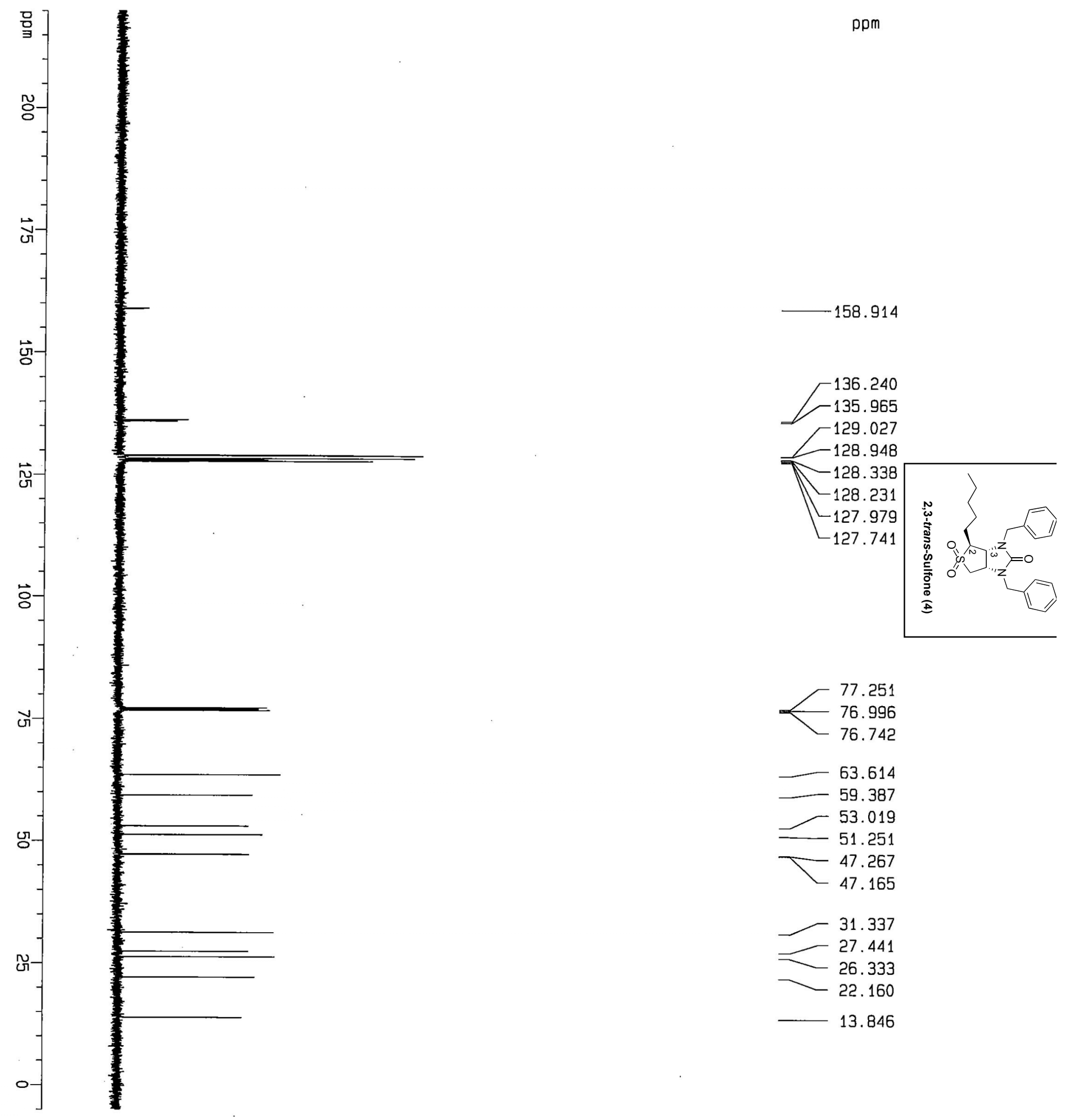


\section{${ }^{1}$ H NMR Spectrum of 2,3-cis-Sulfoxide 6 a}

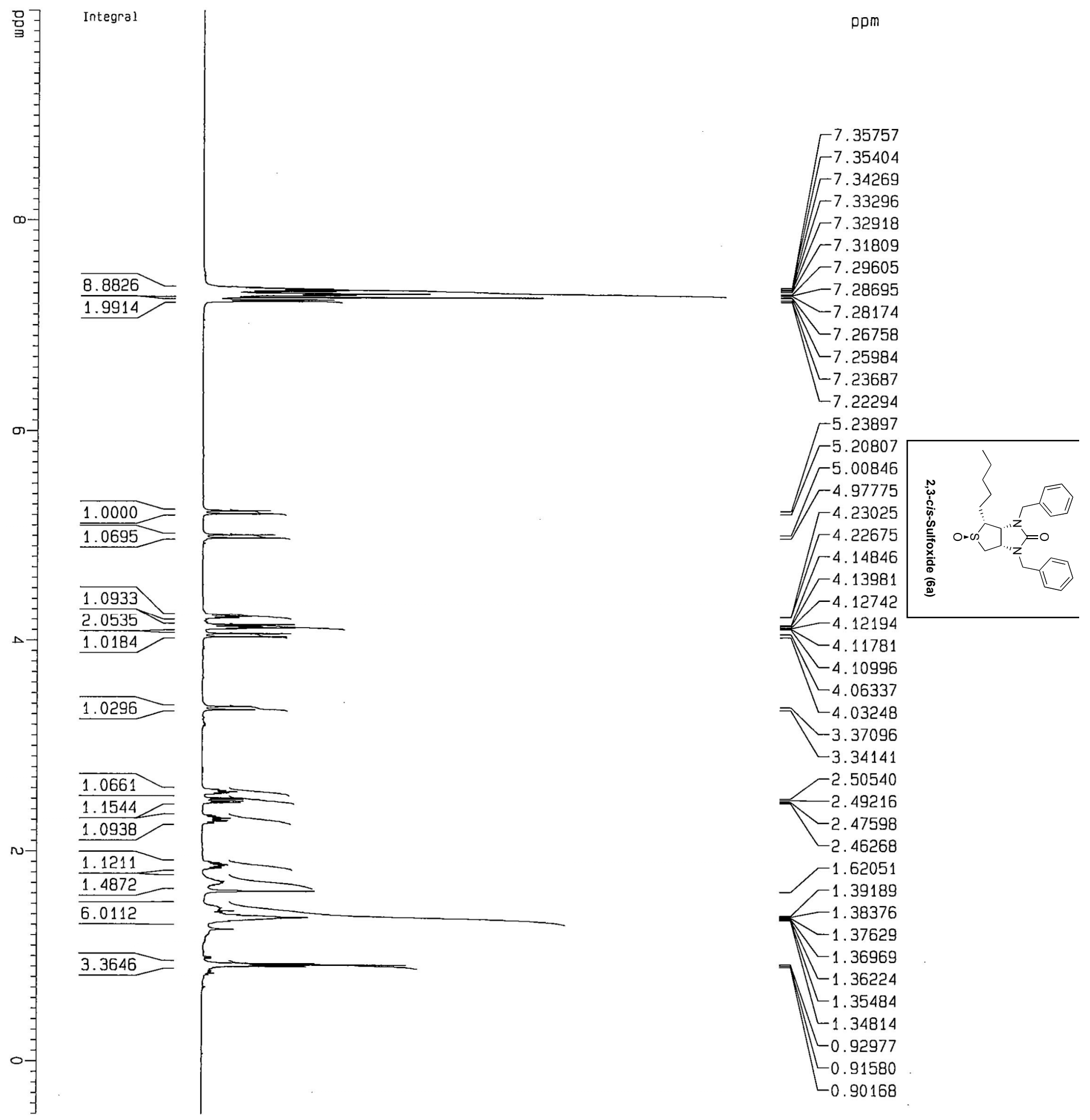




\section{${ }^{13}$ C NMR Spectrum of 2,3-cis-Sulfoxide 6 a}

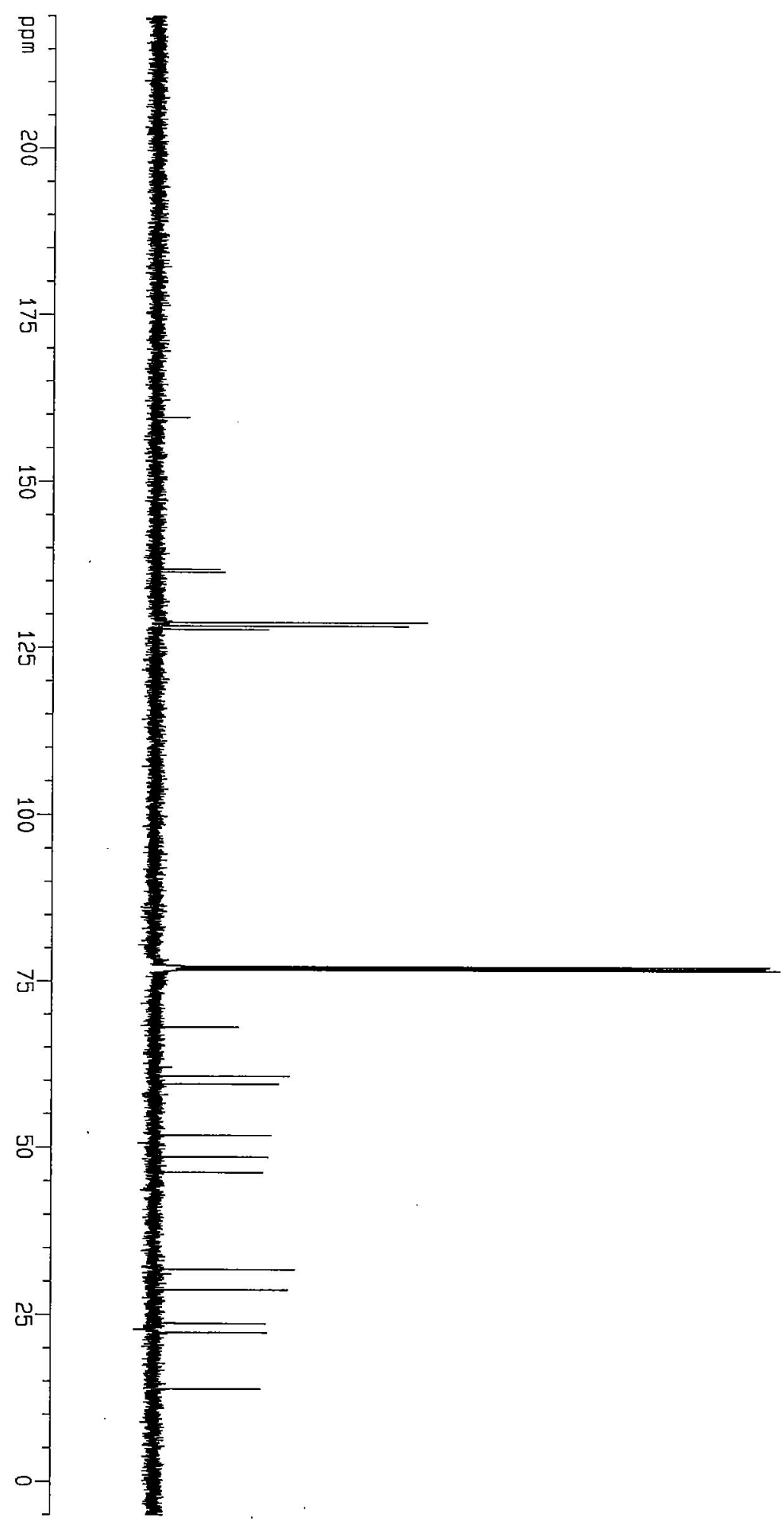

ppm
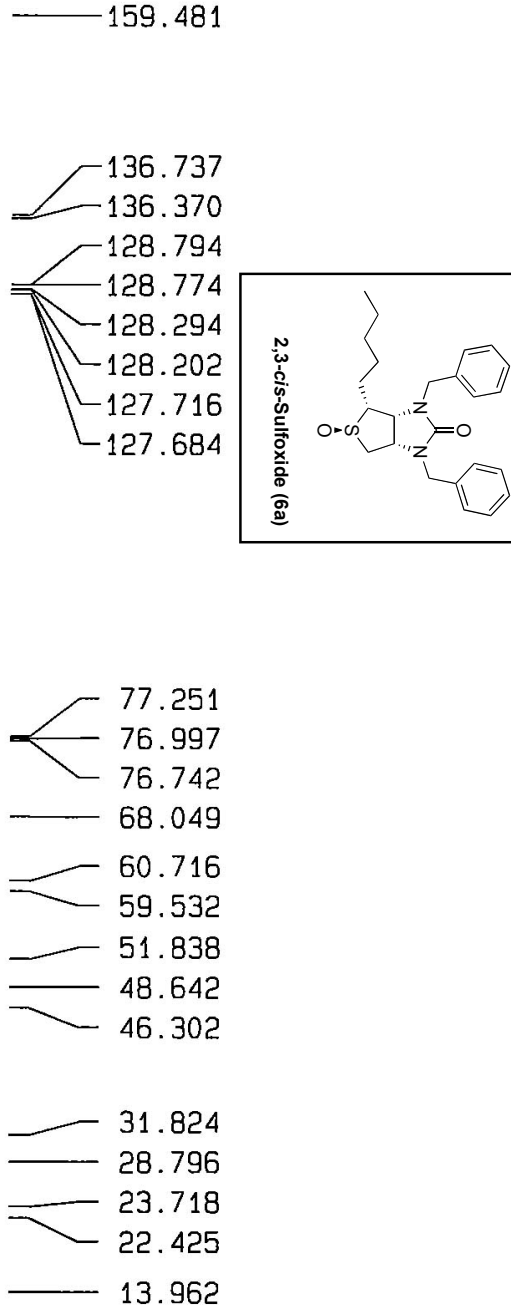


\section{${ }^{1}$ H NMR Spectrum of 2,3-trans-Sulfoxide 6}

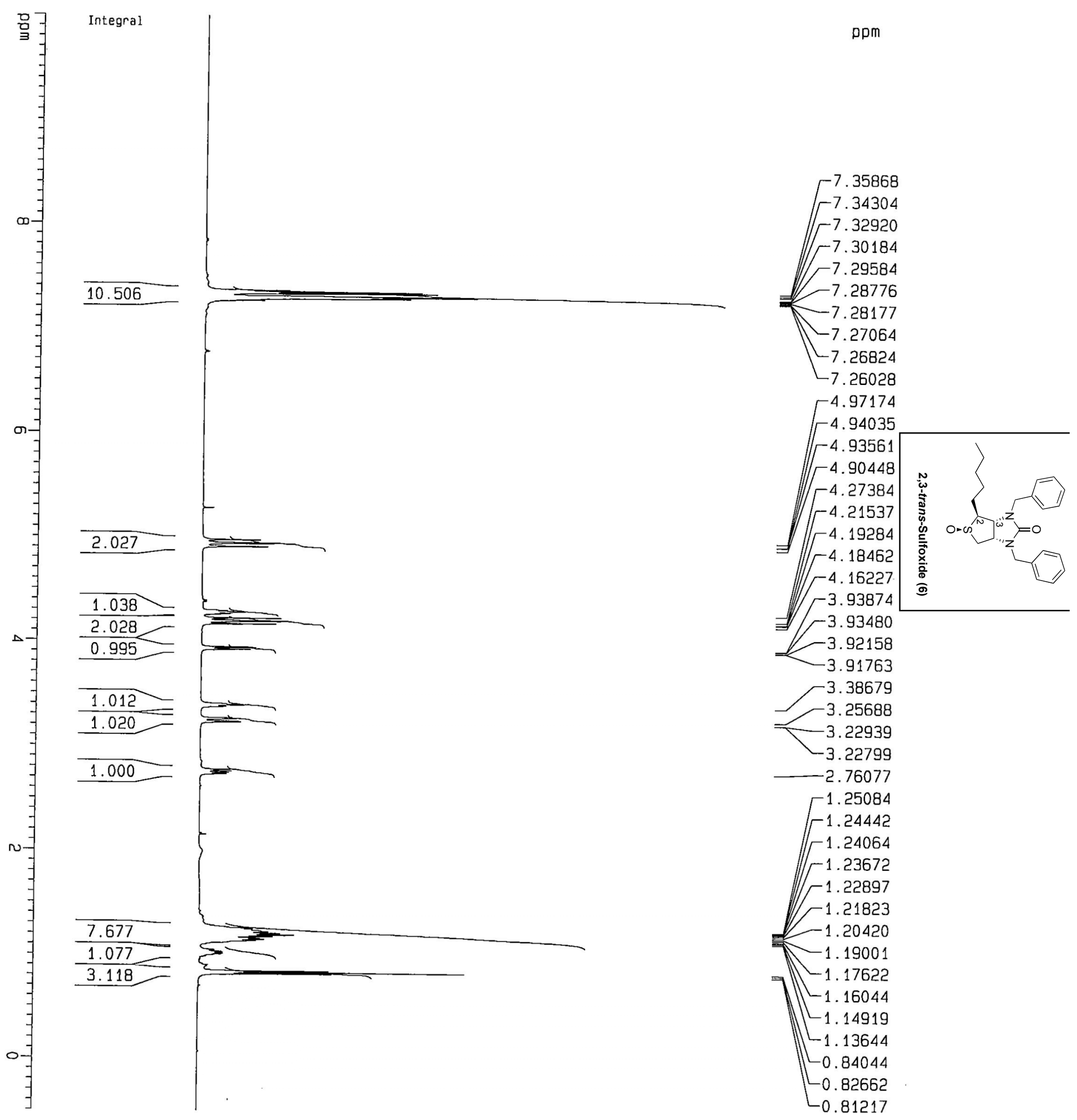




\section{${ }^{13}$ C NMR Spectrum of 2,3-trans-Sulfoxide 6}
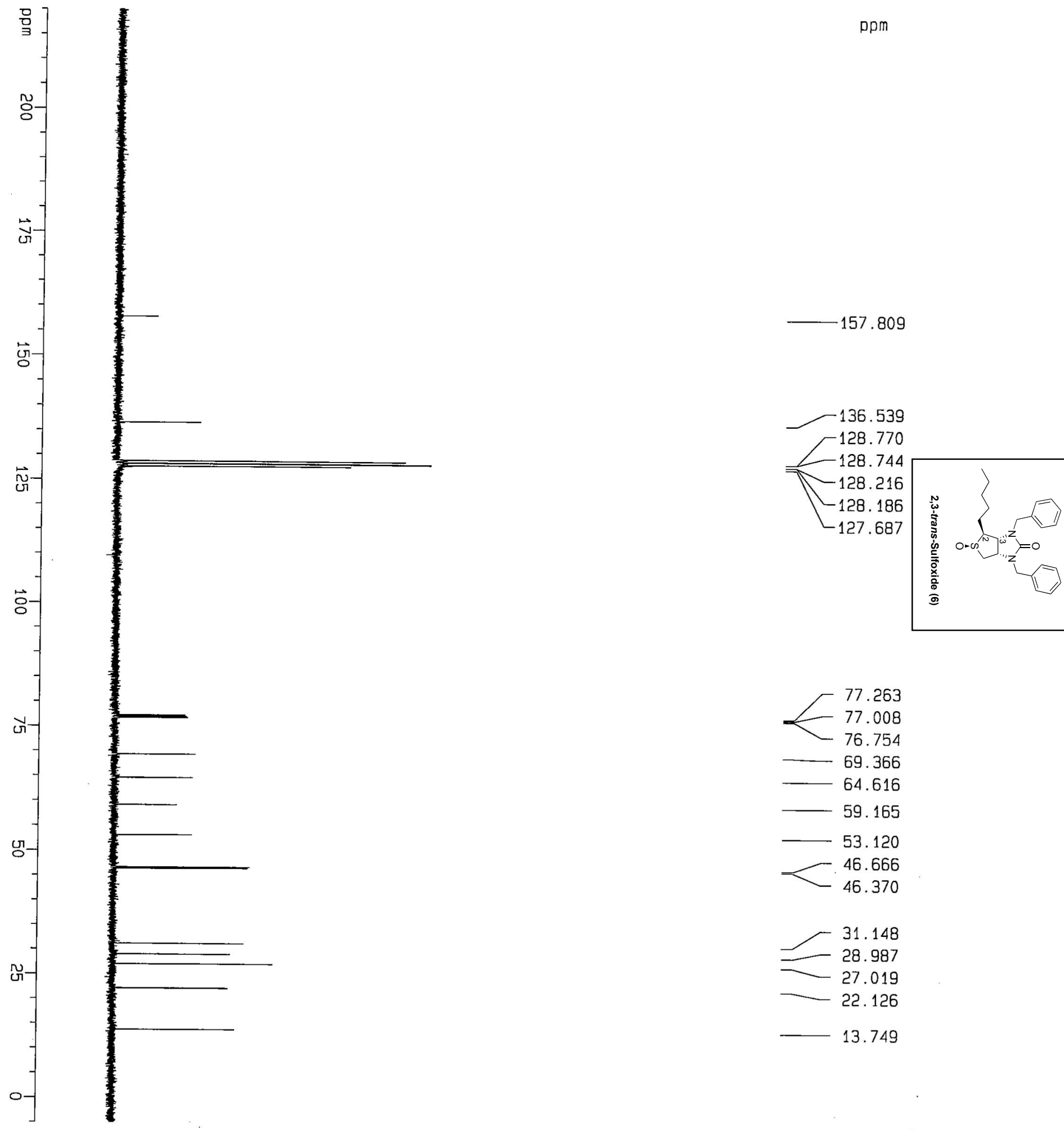


\section{${ }^{1}$ H NMR Spectrum of 2,3-cis-Sulfoxide 5a}

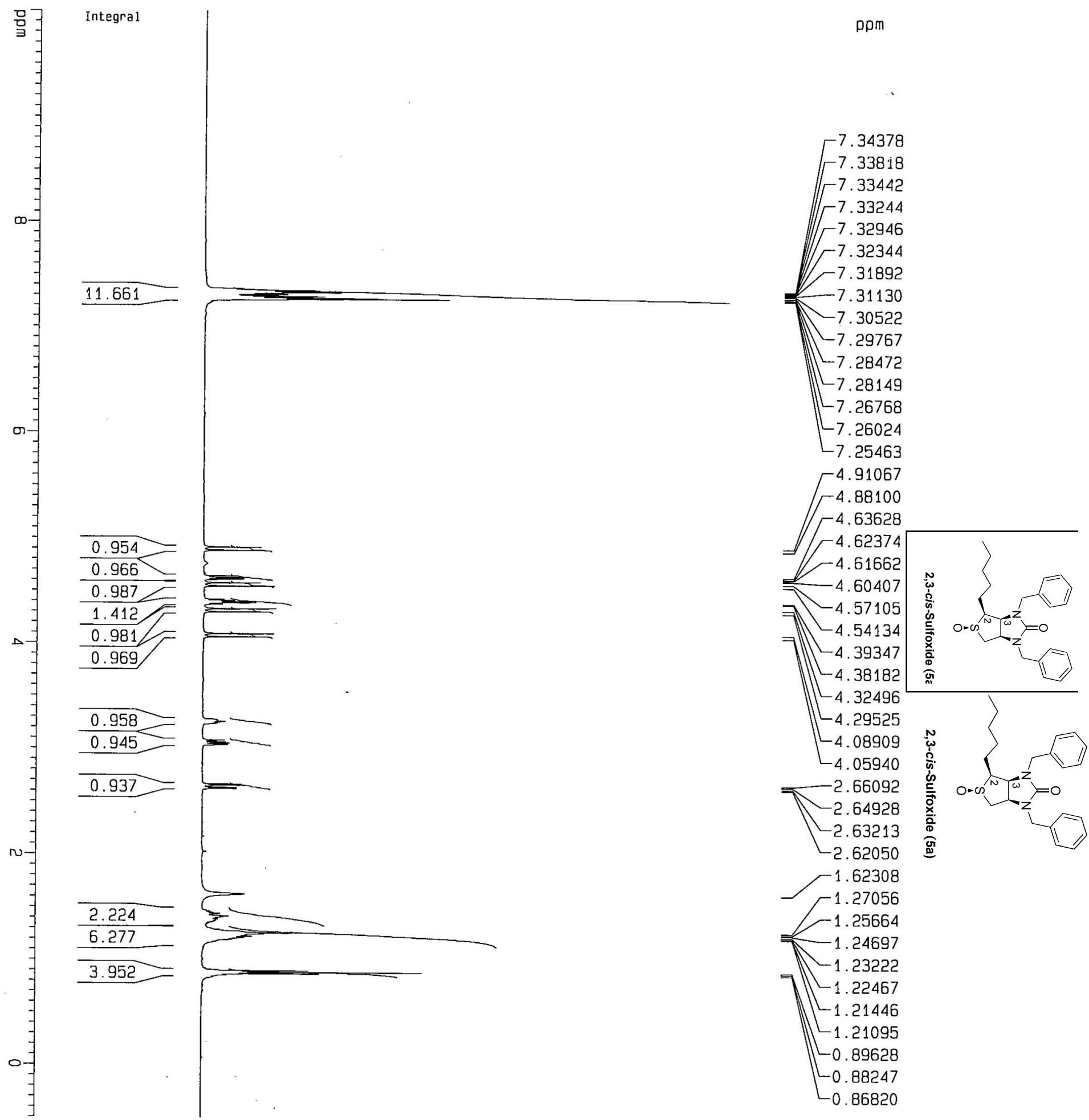




\section{${ }^{13}$ C NMR Spectrum of 2,3-cis-Sulfoxide 5a}
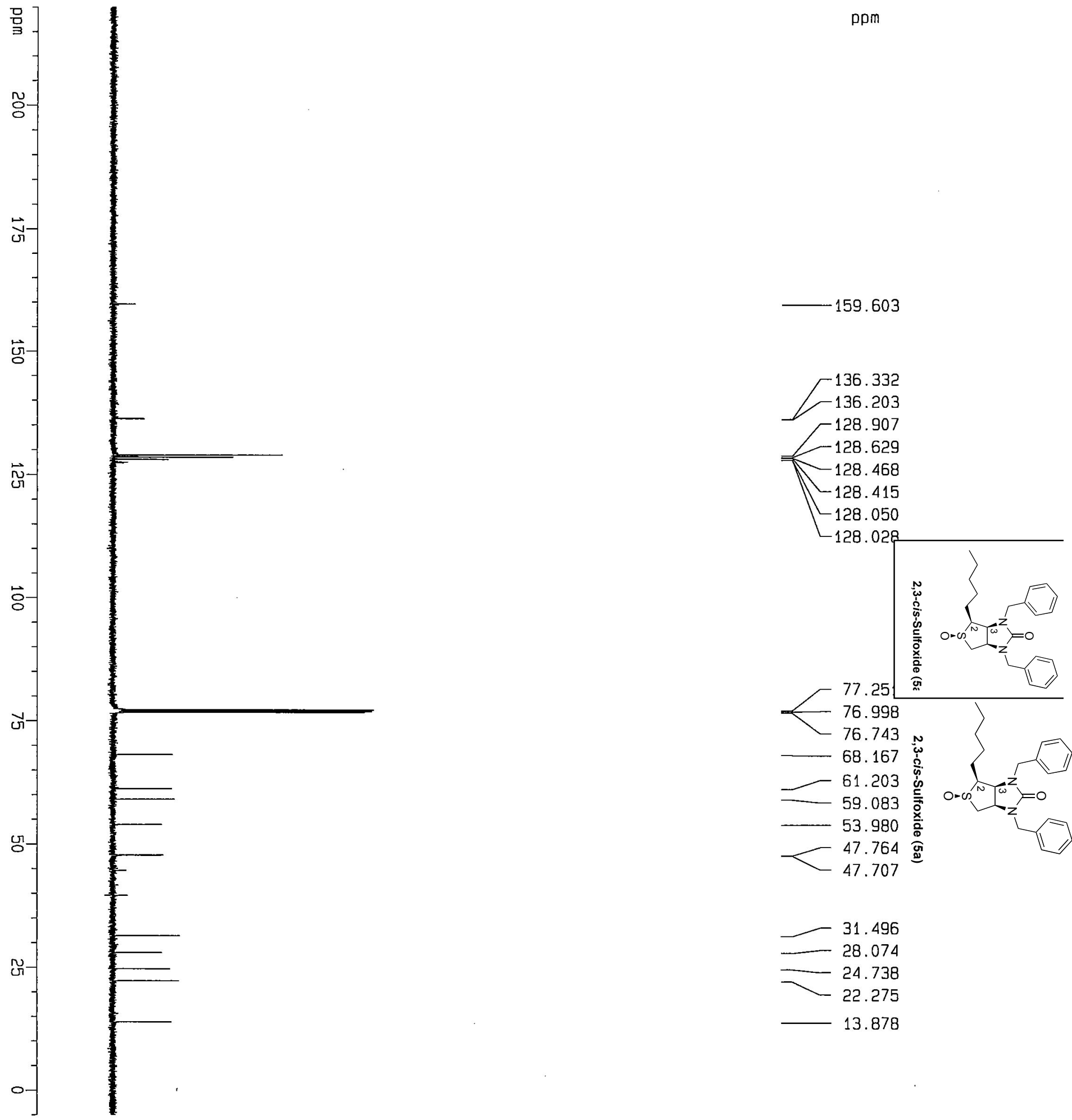


\section{${ }^{1}$ H NMR Spectrum of 2,3-trans-Sulfoxide 5}

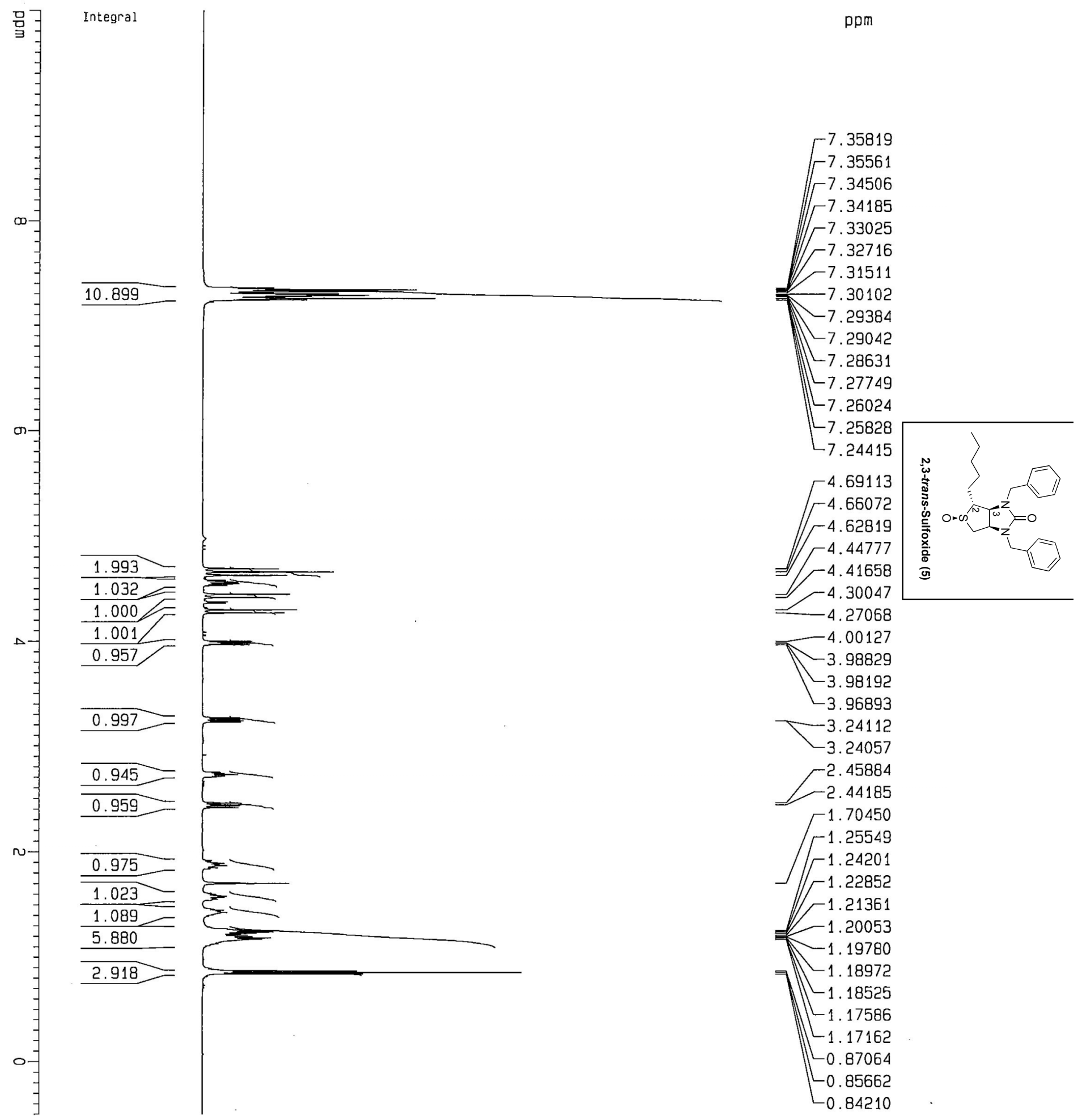




\section{${ }^{13}$ C NMR Spectrum of 2,3-trans-Sulfoxide 5}

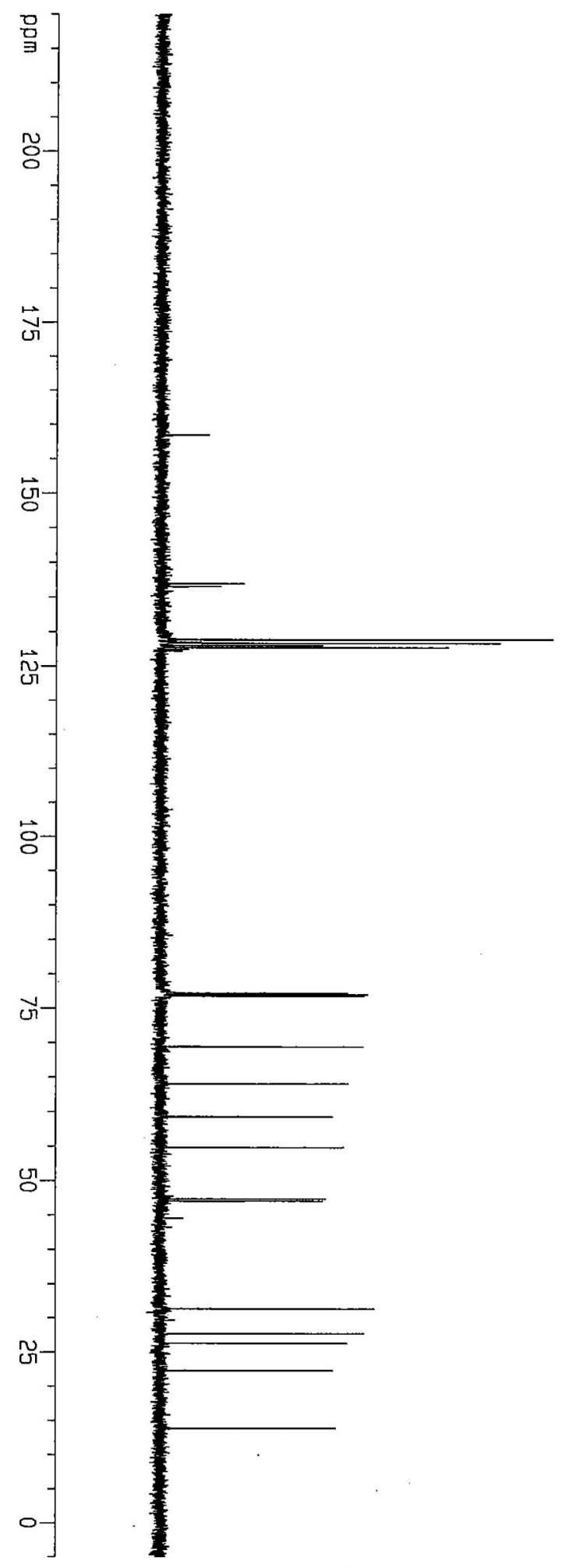

ppm
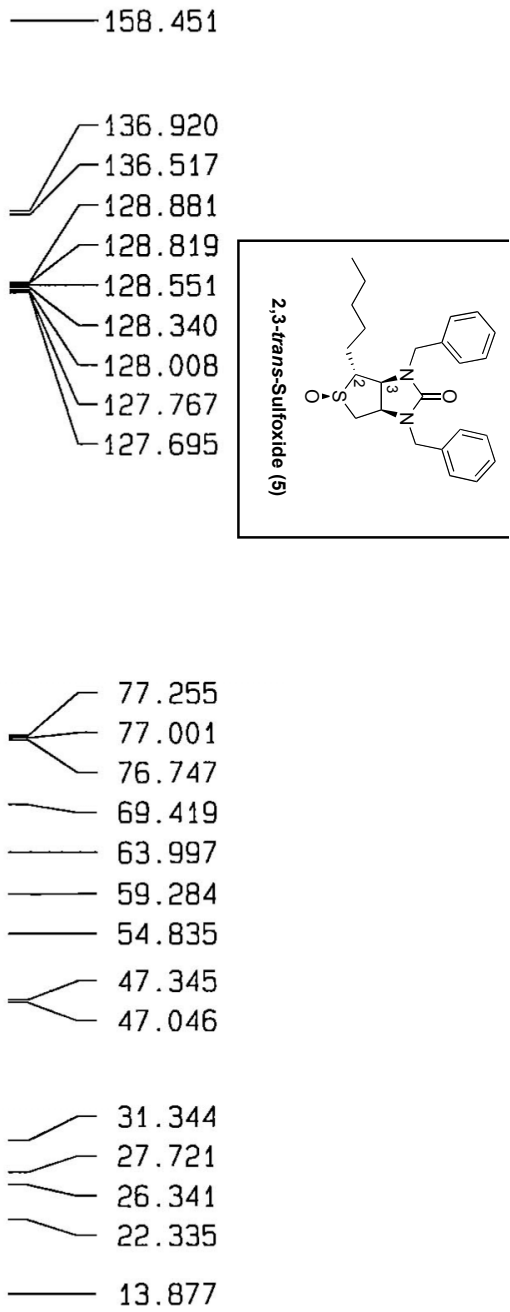


\section{${ }^{1}$ H NMR Spectrum of 2,3-cis-Sulfide 18}

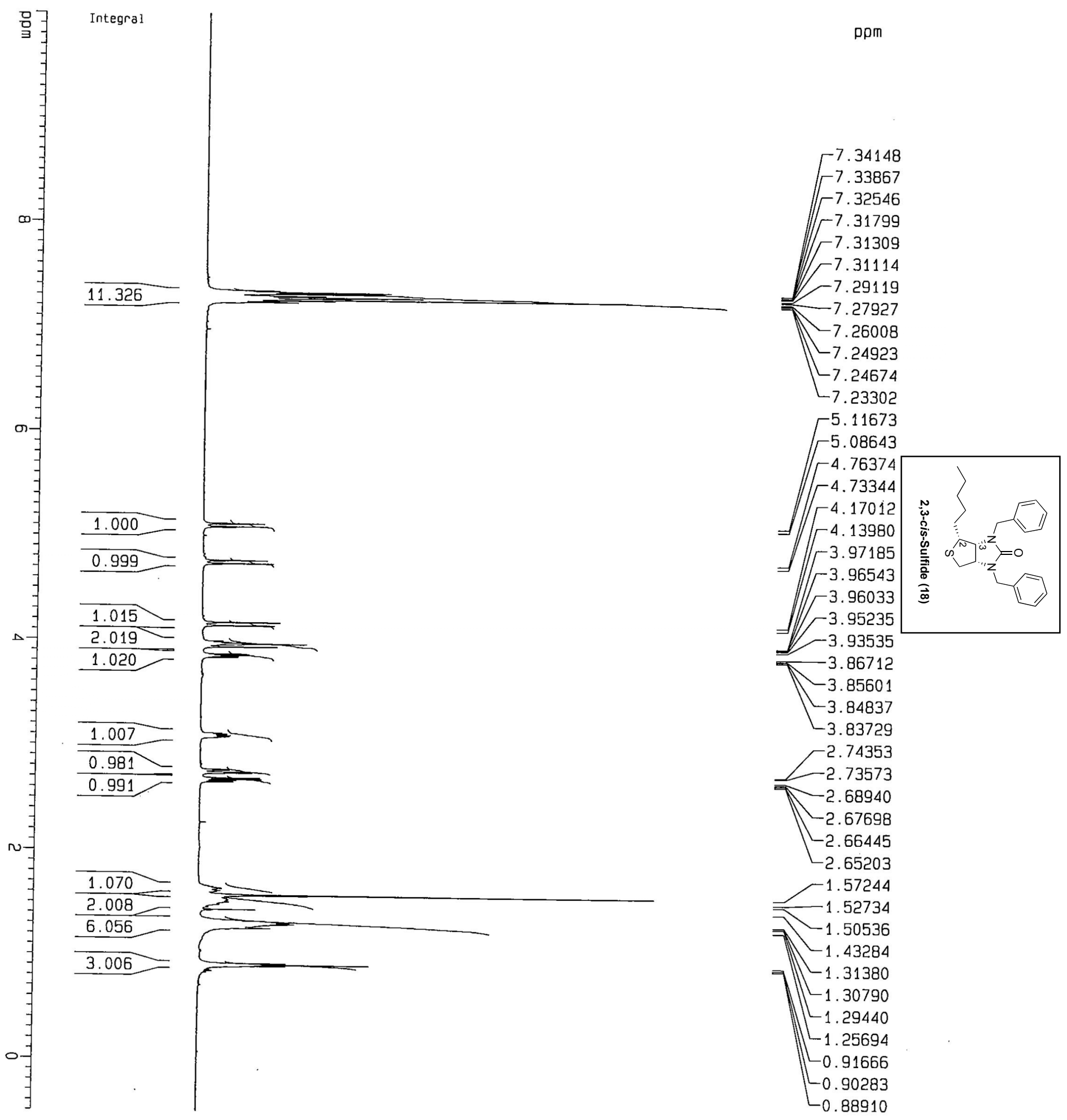




\section{${ }^{13}$ C NMR Spectrum of 2,3-cis-Sulfide 18}
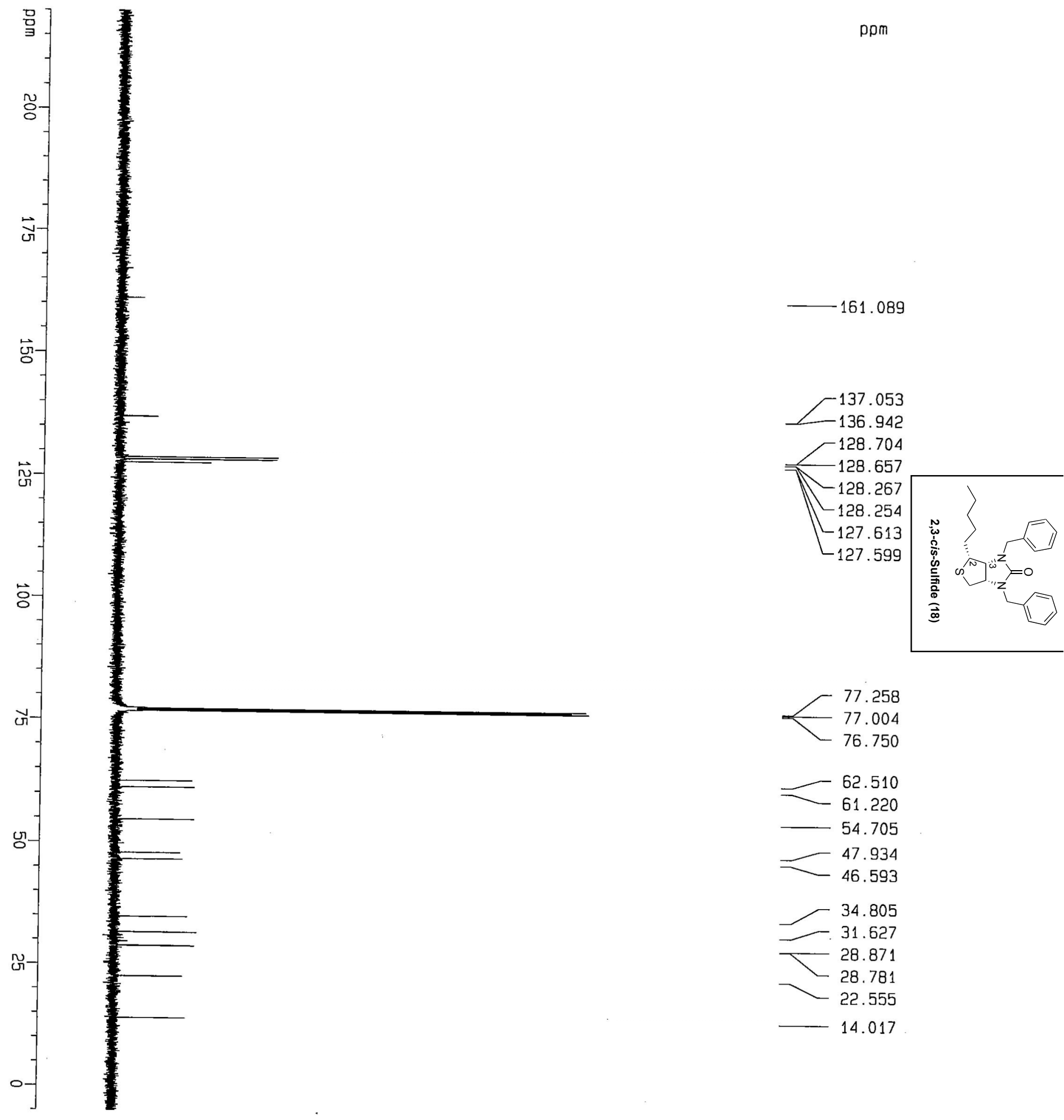


\section{${ }^{1}$ H NMR Spectrum of 2,3-trans-Sulfide 2}

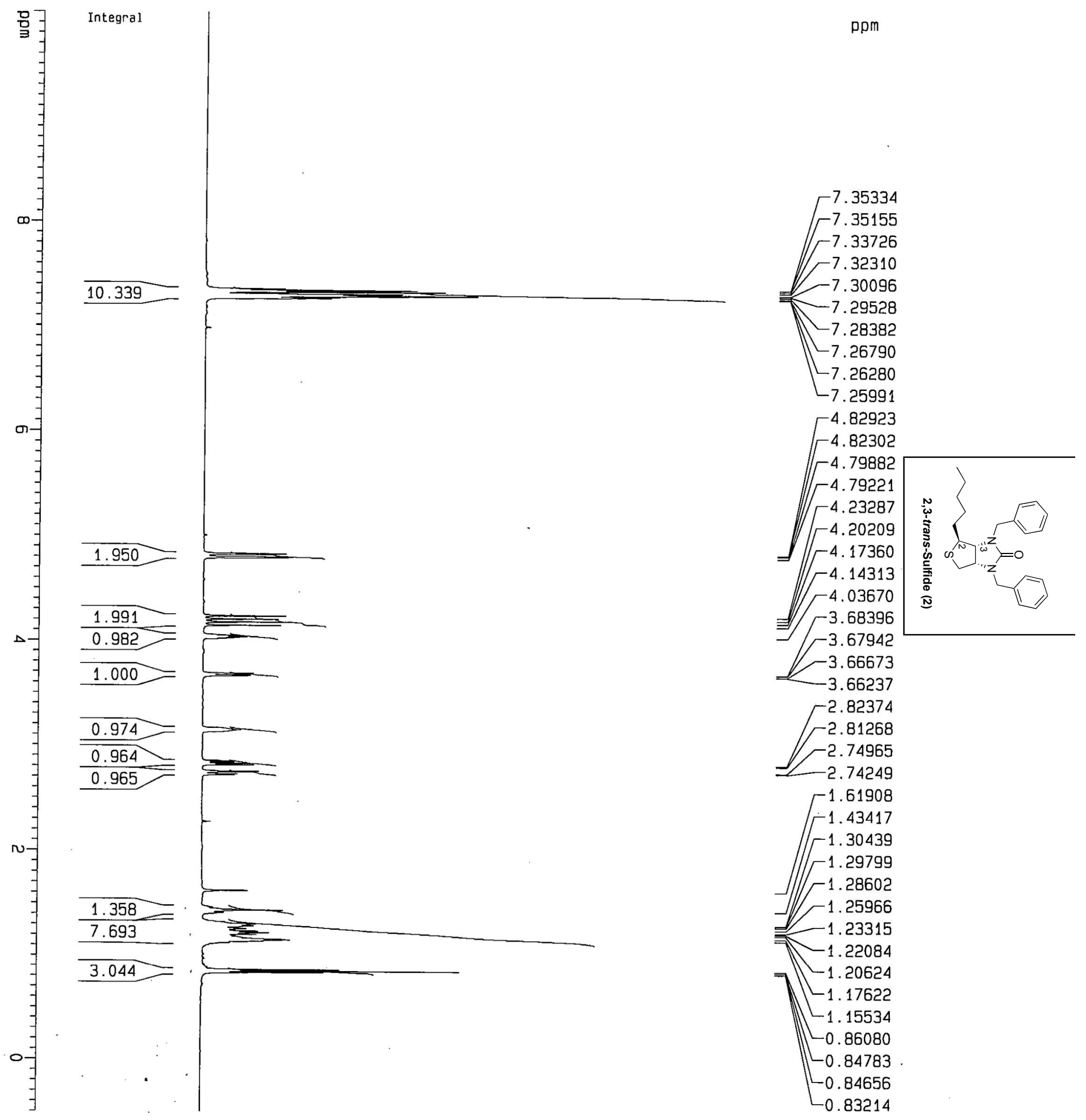




\section{${ }^{13} \mathrm{C}$ NMR Spectrum of 2,3-trans-Sulfide 2}
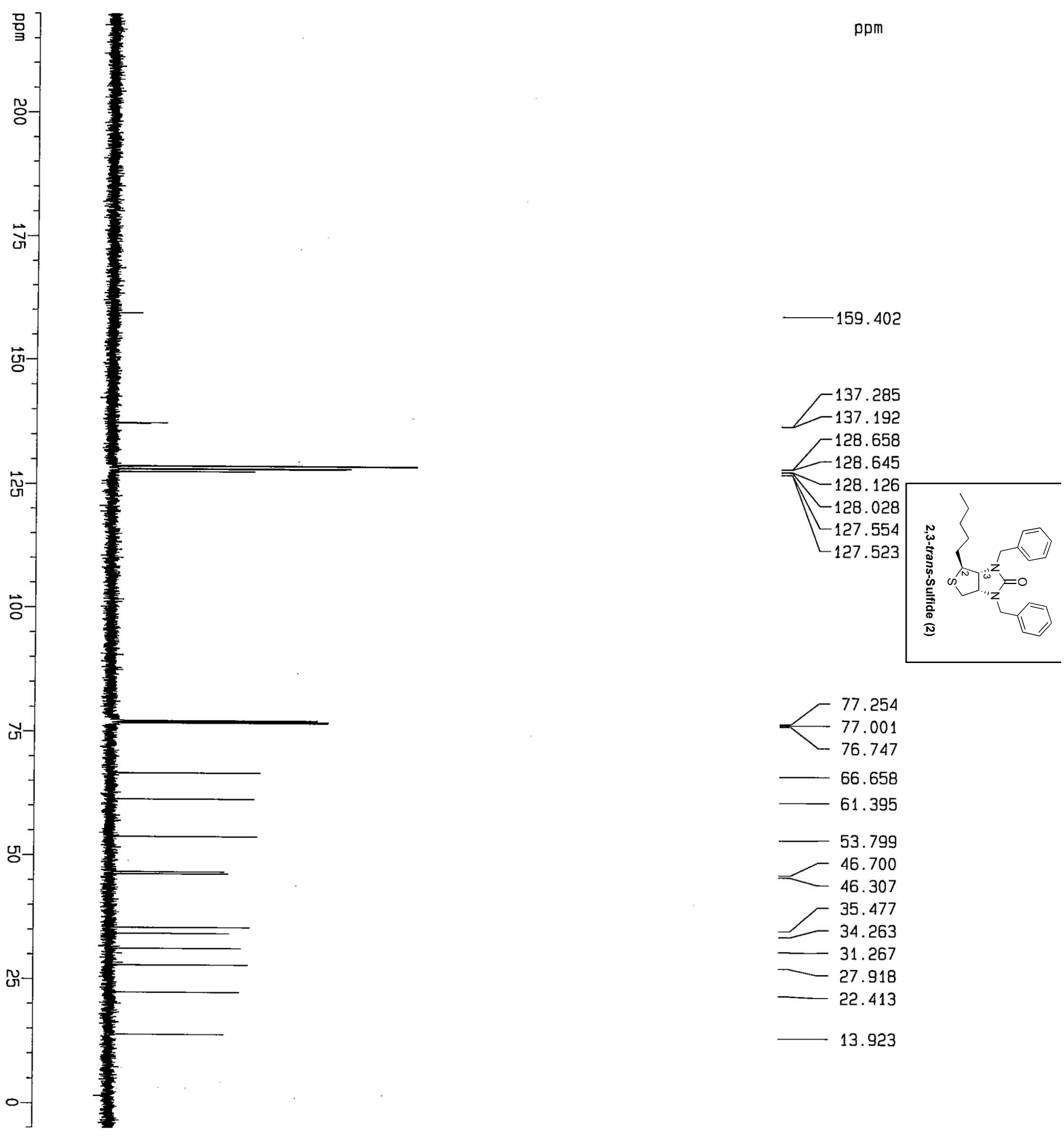